This is a pre-copyedited, author-produced version of an article accepted for publication in Review of Finance following peer review. The version of record Sato Y. (2016). Fund tournaments and asset bubbles. Review of Finance, 20(4), 1383-1426 is available online at: https://doi.org/10.1093/rof/rfv044.

\title{
Fund Tournaments and Asset Bubbles*
}

\author{
Yuki Sato ${ }^{\dagger}$ \\ University of Lausanne and Swiss Finance Institute
}

July 27,2015

\begin{abstract}
This paper studies how fund managers' relative-performance concerns affect their investment strategies in bubble periods. The managers compete for flows that are sensitive to their performance ranking. Severe ranking tournaments with highly convex flow-performance relationship lead managers to ride bubbles to outperform each other, making bubbles long-lived. However, moderate tournaments may lead them to attack bubbles quickly. The results are consistent with the observed cross-sectional variation in funds' investment strategies in bubble periods. Bubble-riding behavior is pronounced if the funds' tournament is too close to call, as interim followers try to catch up while interim leaders try to stay ahead.
\end{abstract}

JEL classification: D82, G14, G23

Key words: asset bubbles, relative performance, fund tournaments, fund flows, synchronization risk, strategic interactions

*This paper was previously circulated under the title "Ranking Concerns of Fund Managers and the Persistence of Bubbles." I am grateful to Margaret Bray, Markus Brunnermeier, Denis Gromb, and Dimitri Vayanos for very helpful discussions. I benefited from suggestions from Burton Hollifield (the editor) and an anonymous referee. For helpful comments, I thank Elias Albagli, Ulf Axelson, Amil Dasgupta, Vincent Fardeau, Daniel Ferreira, Robin Greenwood, Nobuhiro Kiyotaki, Peter Kondor, Stephen Morris, Bob Nobay, Hyun Song Shin, Pietro Veronesi, Paul Woolley, Wei Xiong, and Adam Zawadowski, as well as seminar participants at London School of Economics and Princeton University. Financial support from the Swiss Finance Institute and the Paul Woolley Centre for the Study of Capital Market Dysfunctionality at LSE are gratefully acknowledged. All errors and omissions are my own.

${ }^{\dagger}$ Department of Finance, University of Lausanne, UNIL-Dorigny, Extranef 235, CH-1015 Lausanne, Switzerland. E-mail: yuki.sato@unil.ch 


\section{Introduction}

When analyzing asset bubbles in modern financial markets, it is important to account for institutional investors such as mutual funds or hedge funds because they now represent a large part of the market. ${ }^{1,2}$ Their rapid increase in size may have affected the formation and stabilization of asset prices, as their objectives differ from those of individual investors: while an individual investor simply maximizes her risk-adjusted portfolio return, a fund manager may also aim to perform well relative to her peers. Indeed, fund managers are typically evaluated against each other by investors who find it difficult to distinguish expertise from luck. Various rankings appear in financial media, and there is evidence that fund flows respond to mutual funds' relative performance (Sirri and Tufano 1998). Thus, to attract flows, the managers are naturally concerned with their positions in the ranking table. How do fund managers' relative-performance concerns affect their investment strategies in bubble periods? Do they magnify or dampen bubbles?

To answer these questions, we develop a model à la Abreu and Brunnermeier (2003) (henceforth, AB), in which a bubble persists endogenously as fund managers' "synchronization risk" leads them to defer correcting the mispricing. We depart from AB in assuming that a part of the managers care about their relative performance: their final payoffs are sensitive to the performance ranking through the empirically documented convex flow-performance relationship. The rest of the managers are not ranking-concerned, as in AB. Our setting allows us not only to study the implications of fund managers' ranking concerns for their bubble-riding behavior and the associated persistence of bubbles but also to provide explanations to the observed cross-sectional variation in funds' investment strategies in bubble periods (Dass, Massa, and Patgiri 2008; Greenwood and Nagel 2009).

More specifically, we consider a continuous-time infinite-horizon model of the market for a single asset, in which a continuum of fund managers are fully invested initially. As

\footnotetext{
${ }^{1}$ Investment Company Institute (2014) reports that the number of U.S. households owning mutual funds has increased from 23.4 million in 1990 to 56.7 million in 2013. In 2012, 23\% of households' financial assets are managed by registered investment companies (ETFs, closed-end funds, unit investment trusts, and mutual funds), while it was only $3 \%$ in 1980. Hedge Fund Research reports that in 1990 there were only 610 hedge funds with $\$ 39$ billion of assets globally, whereas in 2013 there are more than 10,000 funds managing $\$ 2,400$ billion of assets.

${ }^{2}$ There are pieces of evidence suggesting that fund managers play important roles in bubble periods. Brunnermeier and Nagel (2004) look directly at hedge fund holdings during the Internet bubble and find that hedge funds were riding instead of attacking the bubble. Griffin, Harris, Shu, and Topaloglu (2011) investigate various players' trading behavior and conclude that institutional investors "drove and burst the tech bubble."
} 
in $\mathrm{AB}$, the managers become sequentially aware that the asset's price is growing beyond its fundamental value. Each manager decides when to attack the bubble by selling the asset, while she does not observe other managers' trades. The bubble bursts when enough managers attack it. Despite the managers' rationality, the bubble persists in equilibrium: since each manager does not know whether she becomes aware of the bubble early or late relative to others, there is a chance that many of her peers have not been aware of the bubble yet, and thus she finds it optimal to ride it by deferring selling the asset in attempting to reap the price appreciation yet avoid being caught in the eventual crash.

We extend $\mathrm{AB}$ by assuming that the managers are ranked based on their investment returns. A part of the managers experience ranking-sensitive outflows: the lower a manager's rank, the larger outflows from her fund, making her final payoff an increasing and convex function of her rank. The assumption captures the convex flow-performance relationship documented in the money management industry (Chevalier and Ellison 1997; Sirri and Tufano 1998). We interpret these ranking-concerned managers as highly incentivized, young managers. Since such a manager has not yet built reputation in the profession, if she performs poorly relative to her peers she is perceived as incompetent and loses her investors. The managers with no ranking concerns are viewed as old ones who have already proved their skill and have steady client investors. This interpretation is motivated by the finding of Chevalier and Ellison (1999) that the termination is more performance-sensitive for younger fund managers.

The model implies that ranking-concerned fund managers have nonmonotonic impacts on bubbles: they may either magnify or dampen bubbles, depending on how much of a winner-take-all situation they are in. If they engage in a severe ranking tournament with highly convex flow-performance relationship, they fuel bubbles by riding for a long time. However, with moderate convexity of the relationship, they help dampen bubbles by attacking them quickly. This nonmonotonicity holds because ranking tournament creates two opposing effects on the managers' risk-taking incentives. The first is the risk-shifting effect: viewing selling out early as a low-risk option (i.e., securing a middle rank) and riding bubbles long as a high-risk option (i.e., gambling between top and worst ranks), the latter becomes more attractive for each manager as convexity of the flow-performance relationship increases. The second is the crash-aversion effect: for fear of getting caught in the crash that triggers large outflows, each manager opts for selling out early to secure a middle rank. Which effect prevails depends on the convexity of the flow-performance relationship. With high convexity, the risk-shifting effect prevails because riding bubbles longer than the peers and winning a top rank is the only way to avoid large outflows. But 
with moderate convexity, the crash-aversion effect may prevail if the bubble is easy to burst under selling pressure, as the managers have a strong incentive to counterbalance the high risk of getting caught in the crash.

Our results imply that, unlike what common wisdom would suggest, (1) fund managers with highly performance-sensitive payoffs may pull out of bubbles quicker than the others, and (2) fund managers' ranking tournament may help stabilize the market by eliminating bubbles quickly. These are in line with Dass, Massa, and Patgiri (2008), who find empirical evidence that the higher the incentives in mutual fund managers' advisory contracts, the lesser these funds invest in bubble stocks. Our model complements their work by offering a theoretical explanation: high-incentive funds may opt for staying away from bubbles as they have strong incentives to avoid outflows triggered by getting caught in the crash.

Our model also implies, under highly convex flow-performance relationship, young managers may ride bubbles longer than the old. This is consistent with the finding of Greenwood and Nagel (2009) that young managers tend to buy bubble stocks more than old ones. Our explanation is as follows. A young manager - evaluated based on relative performance due to the lack of track record - is incentivized to ride bubbles for a long time, even if she is aware of the overpricing, for fear of being perceived as an incompetent manager who missed out on the benefit of swelling bubbles.

To study a more realistic case, we extend the model to allow for a heterogeneity in the managers' interim performance. An exogenous shock making a loss in other asset markets lowers the value of a part of the managers' fund assets at an interim date, but all managers will be evaluated under the same ranking rule eventually. If the tournament is too close to call, i.e., the interim performance gap is small, strategic interactions of the managers may lead to an even longer persistence of bubbles because the interim followers try to catch up while the interim leaders try to stay ahead. But with a large interim performance gap, bubbles may burst quickly as the interim followers give up on catching up while the interim leaders lock in their winning positions.

Our paper is related to the literature on the implications of financial intermediation for bubbles. ${ }^{3}$ Most works in this literature analyze price distortions induced by agency problems. Allen and Gorton (1993) argue that fund managers have an incentive to churn bubbles at the expense of their less informed clients. In Allen and Gale (2000), bubbles are the consequence of risk shifting by investors who borrow from banks. Hong, Scheinkman, and Xiong (2008) develop a model in which a bubble arises as fund managers take excessive

\footnotetext{
${ }^{3}$ See Brunnermeier and Oehmke (2013) for the excellent survey on bubbles and crises.
} 
positions in a tech stock as a signal to investors that they understand the new technology. The agency relationship between fund managers and investors plays an important role in our paper, too. Yet unlike the papers just cited, our paper studies bubbles in light of strategic interactions between heterogeneous fund managers and explains the crosssectional variation in funds' strategies in bubble periods.

Our paper is also related to the papers studying the role of relative performance evaluations in financial markets. Basak and Makarov (2012) analyze strategic interactions among fund managers concerned with fund flows. Unlike their paper, we study the managers' investment behavior in bubble periods and the associated persistence of bubbles. Consistent with our model, DeMarzo, Kaniel, and Kremer (2008) show that bubbles stem from investors trying to keep up with the Joneses, and Palomino (2005) argues that relative performance concerns lead managers to adopt riskier investment strategies. ${ }^{4}$ However, unlike these papers, our model also implies that relative performance concerns may dampen bubbles by leading managers to use safer strategies, depending on the degree of convexity of the flow-performance relationship.

Last, the so-called mutual fund tournament literature is related to our paper. Brown, Harlow, and Starks (1996) find that interim follower mutual funds (i.e., funds whose performance over the first half of the year is below the industry median) tend to increase portfolio risk more than the interim leader funds in the second half of the year (see also Koski and Pontiff 1999; Goriaev, Palomino, and Prat 2003). Our model replicates their finding that followers take more risks than leaders for some parameter values. But we also offer a different prediction: if the funds' interim performance gap is so large that the ultimate winners and losers are already evident at an interim date, the interim followers may give up on catching up and opt for safer strategies.

The paper proceeds as follows. Section 2 presents the model. Section 3 characterizes the equilibrium. Section 4 studies the role of heterogeneous interim performance. Section 5 conducts a robustness check. Section 6 concludes. All proofs are in the Appendix.

\section{Model}

We consider the market for a single asset, in which rational fund managers are fully invested. At some point an exogenous bubble begins, i.e., the asset's price starts to grow beyond its fundamental level. Each manager becomes privately aware of the bubble sooner

\footnotetext{
${ }^{4}$ See also Isaac and James (2000) who report an experimental result indicating that tournament incentives lead to bubble-like price distortions.
} 


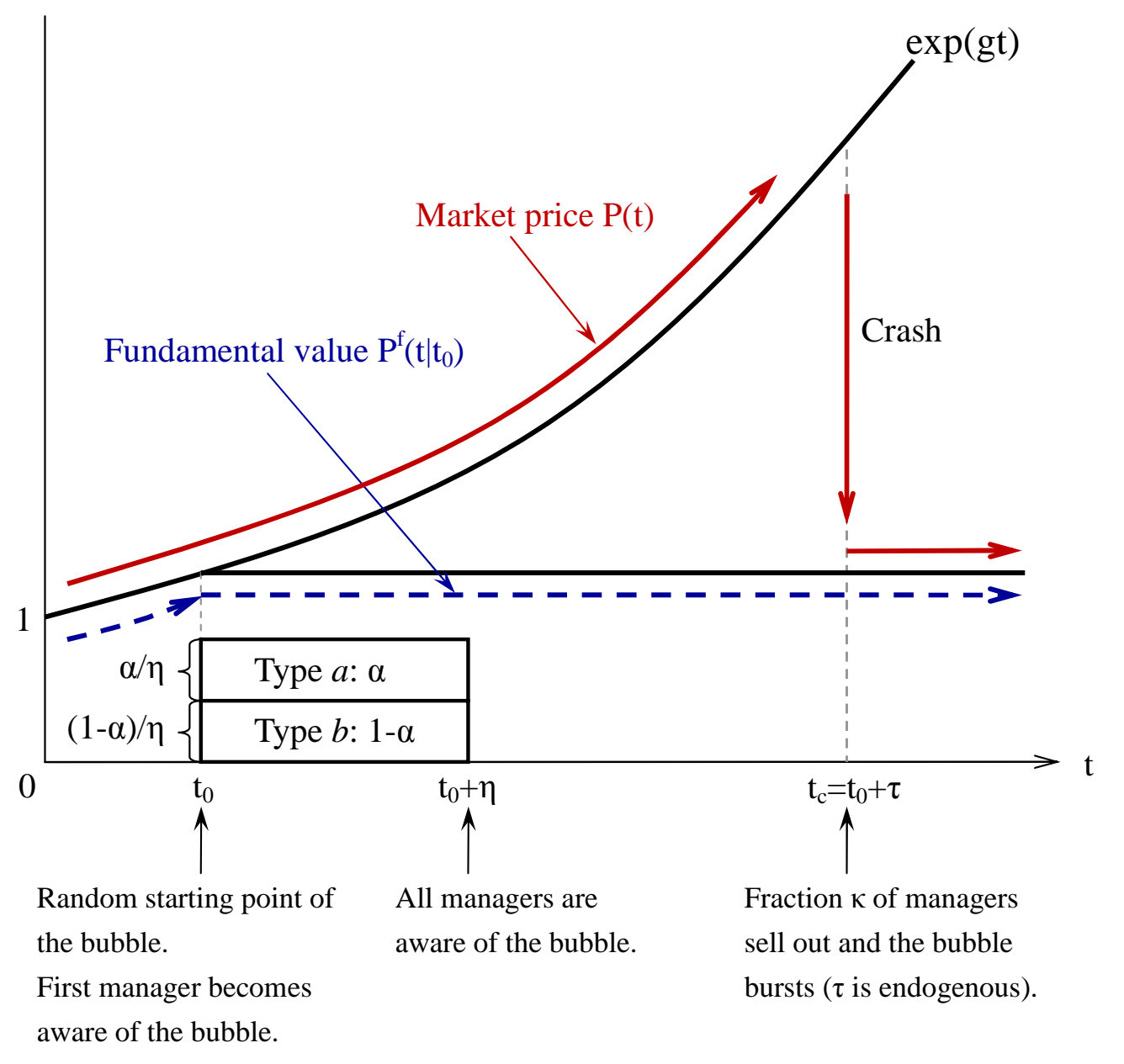

Figure 1: Price path

or later, and decides how long to ride the bubble before selling out. The bubble bursts when enough managers sell out - the crash. After the crash, the managers' performances are evaluated against each other. Some managers' payoffs are sensitive to their relative performance due to convex flow-performance relationship.

\subsection{Price path}

Time $t$ is continuous and runs from 0 to infinity. The time path of the asset's price is modeled as in $\mathrm{AB}$ (Figure 1). The price at $t=0$ is normalized to 1 , and the riskless rate is normalized to 0 . From $t=0$ onwards, the market price $P(t)$, which is publicly observable, grows at a rate $g>0$. The fundamental value of the price, $P^{f}\left(t \mid t_{0}\right)$, coincides with $P(t)$ up to a random time $t_{0}$, but the growth rate of $P^{f}\left(t \mid t_{0}\right)$ drops to 0 thereafter. $P(t)$ is assumed to keep on growing at $g$ even after $t_{0}$. The wedge, $P(t)-P^{f}\left(t \mid t_{0}\right)$, is the bubble 
component of the price. The start time $t_{0}$ of the bubble is unobservable and distributed on $[0, \infty)$ with the cumulative distribution function $\Phi\left(t_{0}\right)=1-\exp \left(-\lambda t_{0}\right)$ with $\lambda>0$. Note that the emergence of a bubble is simply postulated; instead, this model focuses on the bubble's persistence. The crash occurs if and when the cumulative selling pressure by the fund managers is sufficient: specifically, when a fraction $\kappa \in(0,1)$ of managers sell the asset. $^{5}$ Let $t_{c} \equiv t_{0}+\tau$ denote the crash time, where $\tau$ is the endogenous duration of the bubble. After the crash, the price reverts to its fundamental level, i.e., $P(t)=P^{f}\left(t \mid t_{0}\right)$ for $t \geq t_{c}$. Note that the managers do not know $P^{f}\left(t \mid t_{0}\right)$ until the bubble bursts since $t_{0}$ is unobservable. For notational convenience, denote by $\widetilde{P}\left(t_{c}\right) \equiv P^{f}\left(t_{c} \mid t_{c}-\tau\right)=e^{g\left(t_{c}-\tau\right)}$ the fundamental value of the price at the crash time.

To focus on interesting cases, we assume that $g$ is high enough for the bubble to persist for a while, and low enough for the persistence to be finite. The following assumption ensures these.

Assumption 1. $\lambda<g<\frac{\lambda}{1-e^{-\lambda \eta \kappa}}$.

\subsection{Fund managers}

There is a continuum with mass one of risk neutral fund managers, each of whom has invested one dollar of investor capital in the asset at $t=0$. An exogenous proportion $\alpha \in[0,1]$ of the managers are type $a$, who have relative-performance concerns, and the rest are type $b$, who do not have such concerns (detailed later in Section 2.3). The managers become sequentially aware that $P(t)>P^{f}\left(t \mid t_{0}\right)$ : a mass $1 / \eta$ of them becomes privately informed of the bubble in each instant $t \in\left[t_{0}, t_{0}+\eta\right]$. We assume that the manager's type is independent of the time at which she becomes aware of the bubble, so that for all $t \in\left[t_{0}, t_{0}+\eta\right]$, a mass $\alpha / \eta$ of type $a$ and $(1-\alpha) / \eta$ of type $b$ become aware of the bubble. ${ }^{6}$ Each manager decides when to sell the asset. ${ }^{7}$ Her trade is unobservable to the other managers. Importantly, sequential awareness of the bubble and secret trading imply that each manager does not know whether she becomes informed early or late relative to the other managers. These are key assumptions of AB that allow the bubble to persist in

\footnotetext{
${ }^{5}$ To exclude an equilibrium in which all managers do not sell out forever, we assume, as do Abreu and Brunnermeier, that the bubble bursts at time $t_{0}+\bar{\tau}, \bar{\tau}>0$, for an exogenous reason. We assume that $\bar{\tau}$ is very large so that the bubble bursts endogenously.

${ }^{6}$ The crash may occur before some managers have not become informed (i.e., $\tau$ may be smaller than $\eta$ ). In such a case, even uninformed ones become informed at the crash time $t_{c}$.

${ }^{7}$ For simplicity, each manager has no wealth, can neither borrow nor short sell the asset, and if she sells the asset she cannot buy it back.
} 
equilibrium: each manager may think that there is a chance that the fraction of managers who have already been aware of the bubble prior to her is far smaller than the threshold $\kappa$, and may find it optimal to defer attacking the bubble for a while.

We call a manager "manager $k-i$ " if she is type $k \in\{a, b\}$ who becomes aware of the bubble at time $t_{i} \in\left[t_{0}, t_{0}+\eta\right]$. She sells out at time $t_{i}+x_{k, i}$, where $x_{k, i}$ is her endogenous holding period of the asset. Also, we call the managers who sell out after the crash the crashed managers for short.

\subsection{Relative performance objectives}

After the crash, all the managers' performances are evaluated against each other. Specifically, manager $k-i$ is assigned a rank, $\gamma_{k, i}$, defined as the mass of managers whose investment returns from time 0 to the evaluation time are greater than or equal to hers. ${ }^{8}$ Note that $\gamma_{k, i} \in[0,1]$ and a smaller $\gamma_{k, i}$ is better. Each type- $a$ manager's terminal payoff is sensitive to her rank, while it is not the case for type $b$. Specifically, the announcement of $\gamma_{k, i}, k \in\{a, b\}$, at the evaluation time triggers outflows only from type- $a$ funds: a fraction $1-\exp \left(-\theta \gamma_{a, i}\right)$ of investor capital is withdrawn from manager $a$ - $i$, where $\theta \geq 0$ is the flow-performance sensitivity. Equivalently, a fraction $\exp \left(-\theta \gamma_{a, i}\right)$ remains with manager $a-i{ }^{9}$ Each manager's payoff is proportional to the market value of the remaining investor capital at the evaluation time. ${ }^{10}$

Convexity of function $\exp \left(-\theta \gamma_{a, i}\right)$ captures the empirical fact that the relation between fund's past performance and fund flow is positive and convex (Chevalier and Ellison 1997; Sirri and Tufano 1998). Parameter $\theta$ measures the degree of winner-take-all nature of the type- $a$ managers' ranking tournament. That is, if $\theta$ is large, a few high-ranked managers with small $\gamma_{a, i}$ retain a large part of fund assets, whereas mid- and low-ranked ones experience large outflows. Note that $\theta=0$ corresponds to $\mathrm{AB}$ where ranking is irrelevant: our model nests theirs.

\footnotetext{
${ }^{8}$ The assumption that the evaluation takes place after the crash is for simplicity of the analysis. As a robustness check, we study in Section 5 an alternative setting in which the evaluation occurs stochastically, either before or after the crash. We show that the main result carries through in such a setting, and the solution to this section's model is a limit case of that of Section 5. Since the model of Section 5 is significantly less tractable while yielding little additional economic insights, we stick to the simple assumption that the evaluation occurs after the crash.

${ }^{9} \mathrm{~A}$ possible story is as follows. At $t=0$, each fund has a mass one of investors, each of whom invests one dollar of capital in the fund. At the evaluation time, each investor withdraws his capital from a type- $a$ fund if the fund's rank exceeds his "tolerable threshold" $\hat{\gamma}$, where $\hat{\gamma}$ differs for each investor and follows the cumulative distribution function $1-\exp (-\theta \hat{\gamma})$.

${ }^{10}$ This assumption is consistent with the real-world mutual fund industry. Deli (2002) reports that the majority of funds have advisory contracts that depend on a percentage of assets.
} 


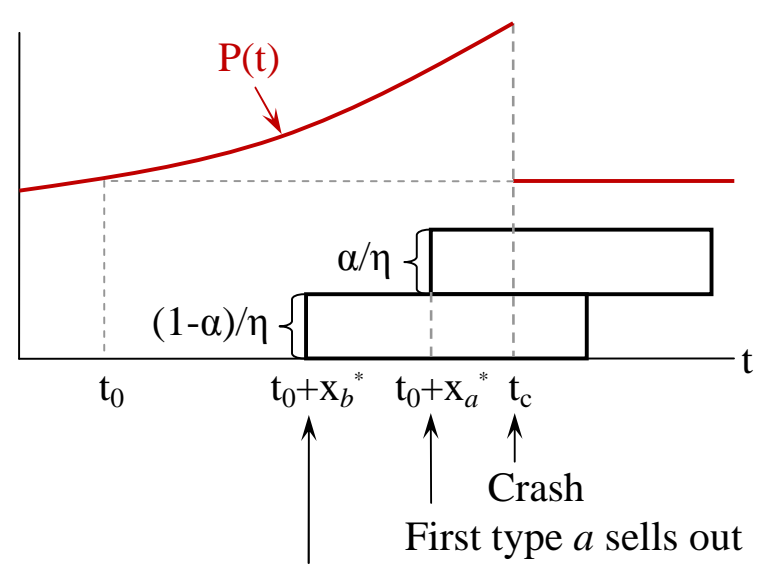

First type $b$ sells out

(a) $x_{a}^{*}>x_{b}^{*}$

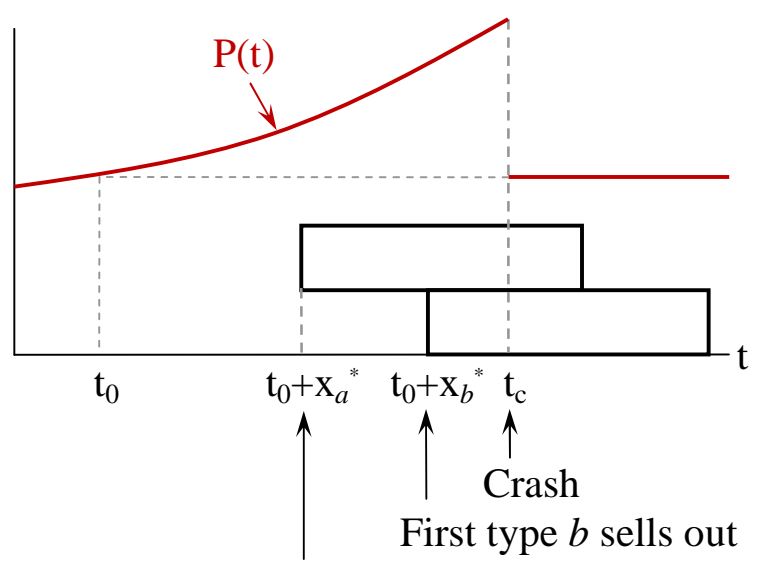

First type $a$ sells out

(b) $x_{b}^{*}>x_{a}^{*}$

Figure 2: Symmetric equilibrium

The managers' types ( $a$ and $b$ ) capture their age or reputation in the money management industry. Each type- $a$ manager is viewed as a highly incentivized, young manager who has not yet built reputation. Due to the lack of track record, investors tend to judge such a manager's skill by relying heavily on her one-shot performance. If she performs poorly, a large fraction of assets is withdrawn, i.e., $\theta$ is positive. On the other hand, each type- $b$ manager is an old one who has already proved her skill and has steady client investors. Such a manager's payoff is not susceptible to the one-time ranking evaluation. This interpretation is consistent with the empirical finding of Chevalier and Ellison (1999) that the termination is more performance-sensitive for younger fund managers.

\section{Persistence of Bubbles}

We look for a symmetric Perfect Bayesian Equilibrium in which each manager correctly believes that all managers of the same type sell out after the same holding period, i.e., $\forall i$ $x_{k, i}=x_{k}^{*}$ for $k \in\{a, b\}$. As illustrated in Figure 2, in equilibrium we have either $x_{a}^{*}>x_{b}^{*}$ or $x_{b}^{*}>x_{a}^{*}$, depending on the parameter values.

\subsection{Manager's rank}

In the symmetric equilibrium, type- $a$ managers start selling out at time $t_{0}+x_{a}^{*}$, after which a mass $\alpha / \eta$ of them sell out in each instant. Likewise, type $b$ start selling out at time $t_{0}+x_{b}^{*}$, after which a mass $(1-\alpha) / \eta$ of them sell out in each instant. The crash 
occurs at time $t_{c}$ where the cumulative selling pressure reaches $\kappa$, which is the sum of the pressures from types $a$ and $b$. Thus, when choosing her holding period $x_{k, i}$ at time $t_{i}$, manager $k-i$ believes that her rank, which depends on the relative position of her sell out time $\left(t_{i}+x_{k, i}\right)$ to the crash time $\left(t_{c}\right)$, is

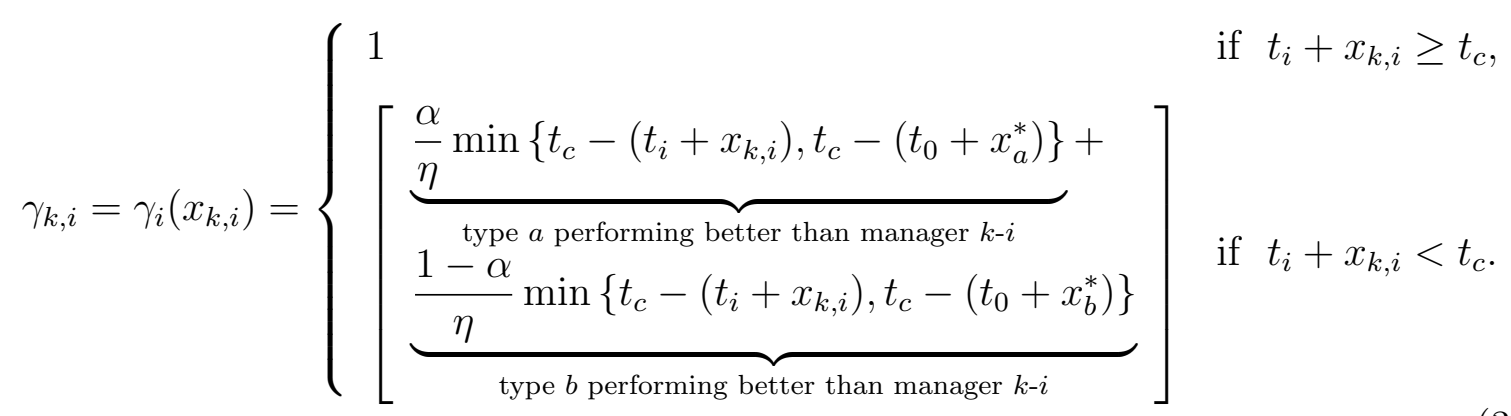

The first line of (3.1) corresponds to the case in which manager $k$ - $i$ ends up selling after the crash $\left(t_{i}+x_{k, i} \geq t_{c}\right)$. In this case, her performance is the worst amongst all the managers, and thus she is assigned the worst rank, $\gamma_{k, i}=1$. The entire expression in the square brackets is her rank if she sells out before the crash $\left(t_{i}+x_{k, i}<t_{c}\right)$. It is the total mass of managers who sell out after manager $k-i$ does so but before the crash occurs. In this case, $\gamma_{k, i}$ depends on how long the bubble persists after manager $k-i$ sells out: the longer the elapsed time until the crash, $t_{c}-\left(t_{i}+x_{k, i}\right)$, the more managers sell out at higher prices than she does, hence the worse her rank becomes. The first line in the square brackets is the mass of type $a$ who perform better than manager $k-i$. If manager $k-i$ sells out after some type $a$ (i.e., if $t_{0}+x_{a}^{*} \leq t_{i}+x_{k, i}<t_{c}$ ), the symmetry of the equilibrium implies that there is a mass $(\alpha / \eta)\left(t_{c}-\left(t_{i}+x_{k, i}\right)\right)$ of type $a$ who sell out at higher prices than manager $k-i$. If manager $k-i$ sells out before any type $a$ (i.e., if $t_{i}+x_{k, i}<t_{0}+x_{a}^{*}$ )-which will not occur in equilibrium but needs to be taken into account in the decision-making stage - then there is a mass $(\alpha / \eta)\left(t_{c}-\left(t_{0}+x_{a}^{*}\right)\right)$ of type $a$ who perform better than her by selling out before the crash. The similar argument applies to her performance relative to type $b$ (the second line in the square brackets). Note that the schedule of ranking is the same for both types; that is, for all $i$, the function $\gamma_{i}(\cdot)$ is independent of $k \in\{a, b\}$. This is because, taking $x_{a}^{*}$ and $x_{b}^{*}$ as fixed, the source of heterogeneity, $\theta$, does not affect the manager's relative performance. ${ }^{11}$

\footnotetext{
${ }^{11}$ In contrast, in Section 4 we study a model in which the managers' heterogeneity is directly relevant to their relative performance, and thus there is a schedule of ranking separately for each type.
} 


\subsection{Manager's objective}

To characterize each manager's objective function, first we need to obtain the distribution of the crash time $t_{c}$. At time $t_{i}$, manager $k-i$ becomes aware of the bubble. From this, she infers that the bubble started no earlier than time $t_{i}-\eta$. Hence her posterior distribution for $t_{0}$, conditional on $t_{i}$, is the truncated distribution with support $\left[t_{i}-\eta, t_{i}\right]$ :

$$
\Phi_{i}\left(t_{0}\right)=\int_{t_{i}-\eta}^{t_{0}} \frac{\mathrm{d} \Phi(v)}{\Phi\left(t_{i}\right)-\Phi\left(t_{i}-\eta\right)}=\frac{e^{\lambda \eta}-e^{\lambda\left(t_{i}-t_{0}\right)}}{e^{\lambda \eta}-1} .
$$

Let $\tau^{*}$ be the equilibrium duration of the bubble, which is a constant that will be determined later. Given that manager $k-i$ believes that the bubble will burst at time $t_{0}+\tau^{*}$, her subjective probability that the bubble will have burst by time $t_{c}$ is

$$
\Pi_{i}\left(t_{c}\right)=\int_{t_{i}-\eta}^{t_{c}-\tau^{*}} \mathrm{~d} \Phi_{i}\left(t_{0}\right)= \begin{cases}\frac{e^{\lambda \eta}-e^{\lambda\left(t_{i}-\left(t_{c}-\tau^{*}\right)\right)}}{e^{\lambda \eta}-1} & \text { for } t_{c} \in\left(t_{i}, t_{i}+\tau^{*}\right) \\ 1 & \text { for } \quad t_{c} \in\left[t_{i}+\tau^{*}, \infty\right)\end{cases}
$$

For $k \in\{a, b\}$, manager $k$-i's problem at time $t_{i}$ is to choose her holding period $x_{k, i} \in$ $[0, \infty)$, taking the other managers' actions $\left(x_{a}^{*}, x_{b}^{*}\right)$ as given, to maximize the expected value of assets under management as of the evaluation time. The objective functions of types $a$ and $b$ are, respectively,

$$
V_{a, i}\left(x_{a, i}\right)=\int_{t_{i}}^{t_{i}+x_{a, i}} e^{-\theta} \widetilde{P}\left(t_{c}\right) \mathrm{d} \Pi_{i}\left(t_{c}\right)+\int_{t_{i}+x_{a, i}}^{\infty} e^{-\theta \gamma_{i}\left(x_{a, i}\right)} P\left(t_{i}+x_{a, i}\right) \mathrm{d} \Pi_{i}\left(t_{c}\right)
$$

and

$$
V_{b, i}\left(x_{b, i}\right)=\int_{t_{i}}^{t_{i}+x_{b, i}} \widetilde{P}\left(t_{c}\right) \mathrm{d} \Pi_{i}\left(t_{c}\right)+\int_{t_{i}+x_{b, i}}^{\infty} P\left(t_{i}+x_{b, i}\right) \mathrm{d} \Pi_{i}\left(t_{c}\right) .
$$

The expectations in (3.4) and (3.5) are taken over the possible crash time $t_{c}$, according to the manager's posterior belief. The first term on the right hand side of (3.4) corresponds to the case in which manager $a-i$ sells out after the crash $\left(t_{i}+x_{a, i} \geq t_{c}\right)$ : her sell-out price is the post-crash price $\widetilde{P}\left(t_{c}\right)$, hence she is ranked lowest $\left(\gamma_{a, i}=1\right)$ and only a fraction $e^{-\theta}$ of fund assets remain with her. The second term corresponds to the case in which she sells out before the crash $\left(t_{i}+x_{a, i}<t_{c}\right)$ : she sells the asset for a bubble price $P\left(t_{i}+x_{a, i}\right)$ and invests the proceeds in the riskless asset thereafter; when the bubble bursts at time $t_{c}$, her rank is given by the second line of (3.1), and so only a fraction $e^{-\theta \gamma_{i}\left(x_{a, i}\right)}$ of fund assets remain with her. Type $b$ 's objective (3.5) is a special case of (3.4) with no outflows, 
i.e., $\theta=0$.

\subsection{Optimization}

Assuming positive solutions, the first-order condition (FOC) for manager $k-i, k \in\{a, b\}$, is $\mathrm{d} V_{k, i}\left(x_{k, i}\right) / \mathrm{d} x_{k, i}=0$. That is,

$$
\frac{\pi_{i}\left(t_{i}+x_{a, i}\right)}{1-\Pi_{i}\left(t_{i}+x_{a, i}\right)}=\frac{\mathrm{E}\left[\mathrm{d}\left(e^{-\theta \gamma_{i}\left(x_{a, i}\right)} P\left(t_{i}+x_{a, i}\right)\right) / \mathrm{d} x_{a, i} \mid t_{c} \geq t_{i}+x_{a, i}\right]}{P\left(t_{i}+x_{a, i}\right)-e^{-\theta} \widetilde{P}\left(t_{i}+x_{a, i}\right)}
$$

for type $a$, and

$$
\frac{\pi_{i}\left(t_{i}+x_{b, i}\right)}{1-\prod_{i}\left(t_{i}+x_{b, i}\right)}=\frac{\mathrm{d} P\left(t_{i}+x_{b, i}\right) / \mathrm{d} x_{b, i}}{P\left(t_{i}+x_{b, i}\right)-\widetilde{P}\left(t_{i}+x_{b, i}\right)}
$$

for type $b$, where $\pi_{i}\left(t_{c}\right) \equiv \mathrm{d} \Pi_{i}\left(t_{c}\right) / \mathrm{d} t_{c}$ is the conditional density of $t_{c}$. For both (3.6) and (3.7), the left hand side is the hazard rate that the crash occurs at $t_{i}+x_{k, i}$, and the RHS is the manager's benefit-cost ratio: the numerator is the marginal benefit from increasing $x_{k, i}$ given that the bubble is still alive when she sells out; the denominator is the loss in payoff if the bubble bursts when she sells out. Type b's FOC (3.7) is identical to that of $\mathrm{AB}$, in which ranking is irrelevant. Unlike (3.7), the RHS's numerator of type $a$ 's FOC (3.6) is given by an expected value, reflecting the fact that the manager's performance relative to her peers is not determined until the random crash time $t_{c}$. That is, each type $a$ 's final payoff depends not only on when she sells the asset, but also on how long other managers keep riding the bubble and thus how long the bubble persists after she has already sold the asset. This contrasts with type $b$, whose final payoff depends solely on his absolute performance that is determined when he sells out.

To see more carefully how type $a$ 's economic incentive differs from that of type $b$, let us study the RHS of (3.6). The numerator implies that there are two channels through which an increase in $x_{a, i}$ increases the type $a$ 's payoff given that she does not get caught in the crash. First, it increases her sell-out price $P\left(t_{i}+x_{a, i}\right)$. Second, it improves her

rank $\gamma_{i}\left(x_{a, i}\right)$ by lowering the time to crash $t_{c}-\left(t_{i}+x_{a, i}\right)$. Specifically, if her rank is $\gamma_{a, i}=\left(t_{c}-\left(t_{i}+x_{a, i}\right)\right) / \eta$ then a marginal increase in $x_{a, i}$ improves $\gamma_{a, i}$ by $1 / \eta$. The impact of this adjustment in $\gamma_{a, i}$ on her payoff is magnified by a factor of $\theta$ through the convex flow-performance relationship. For type $b$, only the first channel is relevant. The denominator of the RHS of (3.6) implies that, ceteris paribus, each type $a$ suffers a larger loss than type $b$ when she gets caught in the crash. This is because the crashed type $a$ not only sells at the lowest price but also experiences large outflows. 


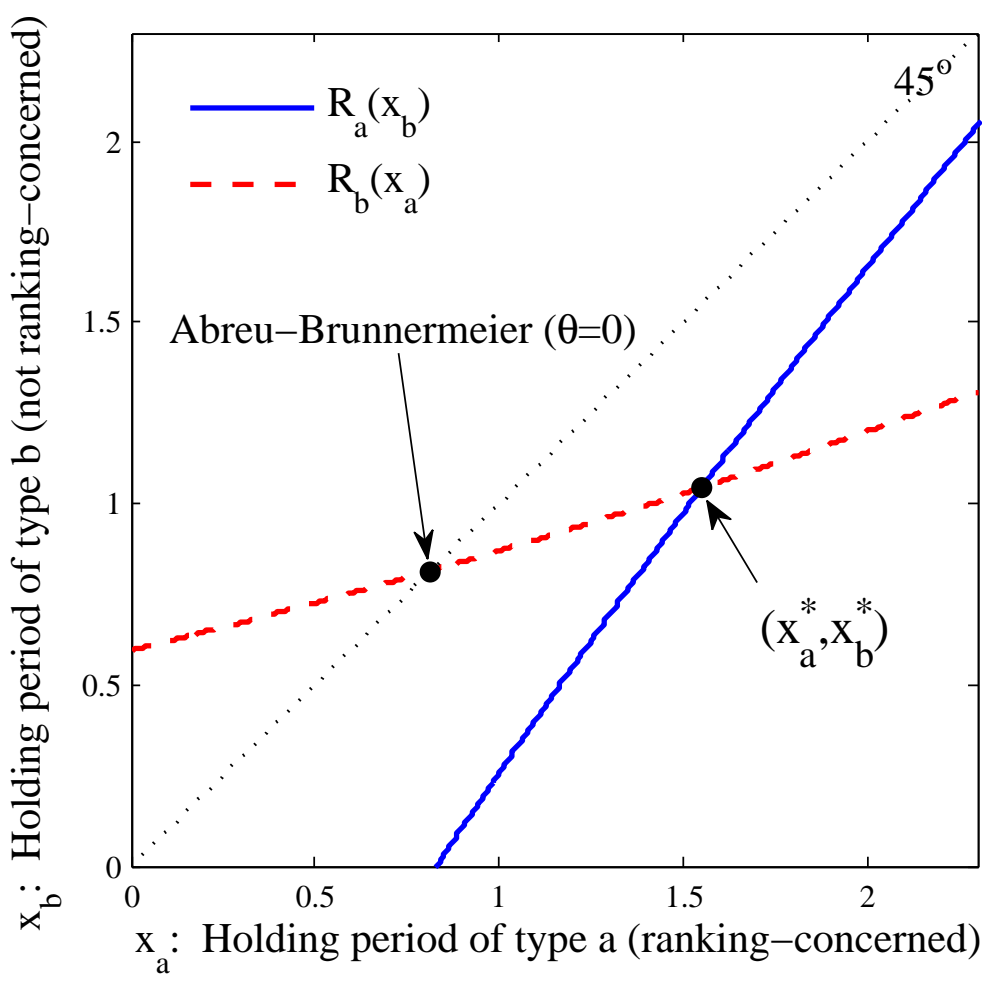

Figure 3: Determination of the symmetric equilibrium. The parameter values used in the figure are $g=0.2, \lambda=0.025, \eta=5, \kappa=0.5, \alpha=0.5$, and $\theta=2.5$.

In sum, compared to type $b$, an increase in $x_{a, i}$ yields an additional marginal benefit to each type $a$ if she sells out before the crash (i.e., improving her rank), but entails a larger loss if she sells after the crash (i.e., experiencing large outflows). Thus, it is ambiguous whether the entire RHS - the benefit-cost ratio - is higher or lower than that of type $b$. As we shall see in Section 3.4, this ambiguity is the reason for type $a$ 's equilibrium holding period to be either longer or shorter than type $b$ 's.

\subsection{Equilibrium}

The symmetric equilibrium is characterized by a pair of holding periods $\left(x_{a}^{*}, x_{b}^{*}\right)$, which is obtained by the following four steps (see Appendix B for details).

1. Taking other managers' symmetric actions $\left(x_{a}, x_{b}\right)$ as given, compute manager $a$ $i$ 's FOC: $\mathrm{d} V_{a, i}\left(x_{a, i}\right) / \mathrm{d} x_{a, i}=0$. This implicitly defines her best response to other managers' arbitrary actions: $R_{a, i}\left(x_{a}, x_{b}\right)$.

2. Imposing the symmetry condition of type $a$ (i.e., $x_{a, i}=x_{a} \forall i$ ), obtain the equilibrium condition for type $a$ : $\left.\left(\mathrm{d} V_{a, i}\left(x_{a, i}\right) / \mathrm{d} x_{a, i}\right)\right|_{x_{a, i}=x_{a}}=0$. This implicitly defines type $a$ 's 
"symmetric best response" to type $b$ 's arbitrary action, denoted by $R_{a}\left(x_{b}\right)$. Formally, $R_{a}(\cdot)$ is defined by $R_{a, i}\left(R_{a}\left(x_{b}\right), x_{b}\right) \equiv R_{a}\left(x_{b}\right) \forall i$.

3. Repeat steps 1 and 2 for type $b$ and obtain type $b$ 's symmetric best response to type a's arbitrary action: $R_{b}\left(x_{a}\right)$.

4. Identify $\left(x_{a}^{*}, x_{b}^{*}\right)$ at the intersection of $R_{a}\left(x_{b}\right)$ and $R_{b}\left(x_{a}\right)$.

Figure 3 illustrates the determination of the equilibrium. The symmetric best-response curves are upward sloping for both types because there is strategic complementarity between the two types. If one type rides the bubble longer, the bubble persists longer ceteris paribus. This, in turn, makes it more beneficial for the other type to ride the bubble longer, as there is a smaller risk of getting caught in the crash. In Figure 3, the equilibrium point is below the 45-degree line, meaning that type $a$ rides the bubble longer than type $b$. Since AB is a special case of our model with $\theta=0$ (that is, all managers are type $b$ ), its solution corresponds to the intersection of $R_{b}\left(x_{a}\right)$ and the 45-degree line.

The following proposition provides the analytical solutions for the managers' equilibrium holding periods $\left(x_{a}^{*}, x_{b}^{*}\right)$ and the corresponding bubble's duration $\tau^{*}$.

Proposition 3.1 (Symmetric equilibrium). Let

$$
\hat{x} \equiv-\frac{1}{g}\left(\ln \left(1-\frac{g+\frac{\theta}{\eta}}{\lambda+\frac{\theta}{\eta}}\left(1-e^{-\left(\lambda+\frac{\theta}{\eta}\right) \eta \kappa}\right)\right)+\theta\right)-\eta \kappa
$$

and let

$$
\left.x_{A B} \equiv \hat{x}\right|_{\theta=0}=-\frac{1}{g}\left(\ln \left(1-\frac{g}{\lambda}\left(1-e^{-\lambda \eta \kappa}\right)\right)\right)-\eta \kappa
$$

denote the solution of Abreu \&6 Brunnermeier (2003).

- Case 1. If $\hat{x}>x_{A B}$, there is a symmetric equilibrium in which each type-a manager rides the bubble longer than type $b$. Their holding periods are, respectively,

$$
\begin{aligned}
& x_{a}^{*}=-\frac{1}{g} \ln \Omega_{1}(\xi)+(1-\alpha) \xi-\eta \kappa \quad \text { and } \\
& x_{b}^{*}=x_{a}^{*}-\xi
\end{aligned}
$$


with constant $\xi>0$ that solves $\Omega_{1}(\xi)=\Psi_{1}(\xi)$, where

$$
\begin{gathered}
\Omega_{1}(\xi) \equiv e^{(1-\kappa) \theta}\left(e^{-\lambda(\eta \kappa+\alpha \xi)}-g \Lambda(\eta \kappa+\alpha \xi, \eta \kappa-(1-\alpha) \xi, 0)\right) \\
-(g-\lambda) e^{\theta}\left(\begin{array}{c}
\Lambda\left(0, \eta \kappa-(1-\alpha) \xi, \frac{\theta}{\eta}\right) \\
+e^{-\theta \alpha\left(\kappa-\frac{(1-\alpha) \xi}{\eta}\right)} \Lambda\left(\eta \kappa-(1-\alpha) \xi, \eta \kappa+\alpha \xi, \frac{\theta(1-\alpha)}{\eta}\right)
\end{array}\right) \\
\Psi_{1}(\xi) \equiv 1-g \Lambda(0, \eta \kappa+\alpha \xi, 0) \\
\text { and } \Lambda(X, Y, Z) \equiv \frac{e^{-(\lambda+Z) X}-e^{-(\lambda+Z) Y}}{\lambda+Z}
\end{gathered}
$$

- Case 2. If $\hat{x} \leq x_{A B}$, there is a symmetric equilibrium in which each type-a manager sells out earlier than type $b$. Their holding periods are, respectively,

$$
\begin{aligned}
& x_{a}^{*}=-\frac{1}{g} \ln \Omega_{2}(\zeta)-(1-\alpha) \zeta-\eta \kappa \quad \text { and } \\
& x_{b}^{*}=x_{a}^{*}+\zeta
\end{aligned}
$$

with constant $\zeta \geq 0$ that solves $\Omega_{2}(\zeta)=\Psi_{2}(\zeta)$, where

$$
\begin{aligned}
& \Omega_{2}(\zeta) \equiv e^{(1-\kappa) \theta-\lambda(\eta \kappa+(1-\alpha) \zeta)} \\
& -(g-\lambda) e^{\theta}\left(\begin{array}{c}
\Lambda\left(0, \eta \kappa-\alpha \zeta, \frac{\theta}{\eta}\right) \\
+e^{-\theta(1-\alpha)\left(\kappa-\frac{\alpha \zeta}{\eta}\right)} \Lambda\left(\eta \kappa-\alpha \zeta, \eta \kappa+(1-\alpha) \zeta, \frac{\theta \alpha}{\eta}\right)
\end{array}\right) \\
& \text { and } \Psi_{2}(\zeta) \equiv 1-g \Lambda(0, \eta \kappa-\alpha \zeta, 0)
\end{aligned}
$$

- In both cases, the bubble's duration is $\tau^{*}=\eta \kappa+\alpha x_{a}^{*}+(1-\alpha) x_{b}^{*}$.

Two cases are presented in Proposition 3.1 because it may be either $x_{a}^{*}>x_{b}^{*}$ (case 1) or $x_{a}^{*} \leq x_{b}^{*}$ (case 2 ) in equilibrium, depending on the relative size of $\hat{x}$ and $x_{A B}$. Note that $\hat{x}=\left.x_{a}^{*}\right|_{\alpha=1}$ and $x_{A B}=\left.x_{b}^{*}\right|_{\alpha=0}$; that is, $\hat{x}$ (resp., $x_{A B}$ ) is the equilibrium holding period that would be obtained if there were only type $a$ (resp., type $b$ ) in the economy. Thus, Proposition 3.1 states that each type $a$ rides the bubble longer than type $b$ if and only if the managers in the only-type- $a$ economy would ride bubbles longer than those in the onlytype- $b$ economy (i.e., $x_{a}^{*}>x_{b}^{*}$ iff $\left.x_{a}^{*}\right|_{\alpha=1}>\left.x_{b}^{*}\right|_{\alpha=0}$ ), which is an intuitive result. Proposition 3.1 also states that the bubble's equilibrium duration $\tau^{*}$ is linear in the weighted average of $x_{a}^{*}$ and $x_{b}^{*}$, with the weight being the mass of the respective type. 


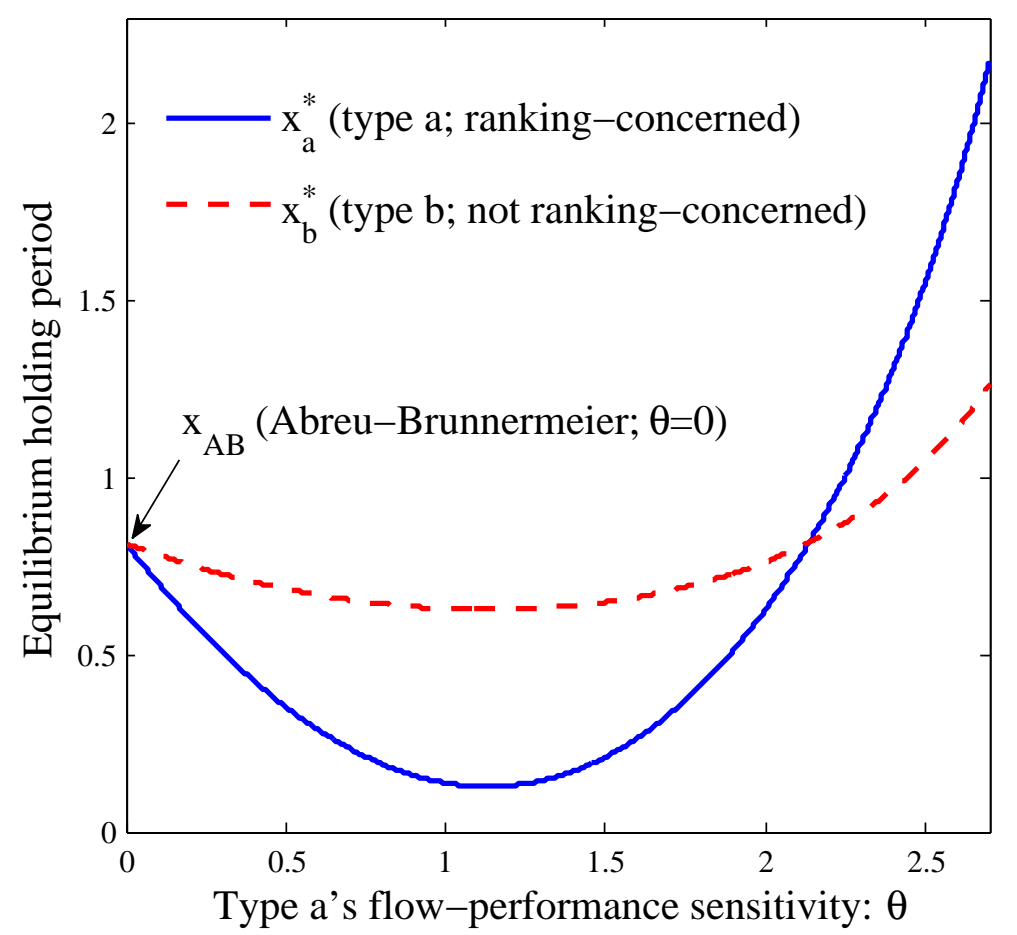

Figure 4: Equilibrium holding periods $\left(x_{a}^{*}, x_{b}^{*}\right)$ as functions of type $a$ 's flow-performance sensitivity $\theta$. The parameter values used in the figure are the same as those of Figure 3.

How do relative-performance concerns alter the managers' actions and thus affect the bubble's duration? The next corollary shows that the answer depends on the level of $\theta$.

Corollary 3.1 (Flow-performance sensitivity and bubble riding). If $\kappa$ is small enough, type-a managers' flow-performance sensitivity $\theta$ has nonmonotonic effects on the equilibrium holding periods $\left(x_{a}^{*}, x_{b}^{*}\right)$. We have $x_{a}^{*}=x_{b}^{*}=x_{A B}$ for $\theta=0, x_{a}^{*}<x_{b}^{*}<x_{A B}$ for small $\theta>0$, and $x_{a}^{*}>x_{b}^{*}>x_{A B}$ for large $\theta>0$.

Corollary 3.1 is illustrated in Figure 4, which plots $x_{a}^{*}$ and $x_{b}^{*}$ as functions of $\theta$. It shows that $x_{a}^{*}$ is U-shaped in $\theta$ : while it equals $x_{A B}$ for $\theta=0$, it is significantly lower than $x_{A B}$ for intermediate levels of $\theta$, and it is much higher than $x_{A B}$ for large $\theta$. Interestingly, not only $x_{a}^{*}$ but also $x_{b}^{*}$ is U-shaped in $\theta$ because type $a$ 's actions "spill over" to type $b$. The intuition is as follows. Since type $b$ have no ranking concerns, they would choose $x_{A B}$ if they were the only players in the market. But, due the presence of type $a$, the type $b$ 's action tilts toward the type $a$ 's in equilibrium: if each type $a$ rides the bubble long (resp., sells out early), the bubble is likely to persist long (resp., short) ceteris paribus, which leads each type $b$ to ride it longer (resp., sell out earlier) too. Thus, the model implies that ranking-concerned fund managers may either fuel or dampen asset bubbles, depending on 
how much of a winner-take-all situation they are in. If they engage in a severe ranking tournament with highly convex flow-performance relationship, they fuel bubbles by riding them long. However, with moderate convexity of the flow-performance relationship, funds' ranking tournament leads them to attack and eliminate bubbles quickly.

Why is $x_{a}^{*}$ nonmonotonic in $\theta$ ? It is intuitive - and perhaps in line with common wisdom - that $x_{a}^{*}$ increases with $\theta$ for large $\theta$. This is caused by the risk-shifting effect: a rise in $\theta$ convexifies each type $a$ 's objective function (3.4) and encourages her to take a riskier action, i.e., choosing larger $x_{a, i}$. Viewing selling out early as a low-risk-low-return option, i.e., securing a middle rank, and riding the bubble long as a high-risk-high-return option, i.e., gambling between top and worst ranks, the latter becomes more attractive for each manager as convexity of the flow-performance relationship increases. Then, why does $x_{a}^{*}$ decrease with $\theta$ for small $\theta$ ? It is because of the crash-aversion effect. In the tournament setting, the crashed managers are far worse off than high- and middle-ranked managers who sell out before the crash because they not only sell at the lowest post-crash price but also experience large outflows due to their worst rank. Thus, for fear of getting caught in the crash, the managers opt for selling out early to secure a middle rank.

In sum, the tournament setting creates two opposing effects on type a's decisions. First, it magnifies their desire to win a top rank, encouraging them to ride bubbles longer to outperform each other: the risk-shifting effect. Second, it also magnifies their incentive to avoid outflows, leading them to sell out earlier: the crash-aversion effect. Which effect prevails depends on $\theta$. If $\theta$ is very large, the risk-shifting effect prevails. Since not only the crashed managers but also early sellers experience large outflows in such a case, riding the bubble longer than the peers and winning a top rank is the only way to avoid large outflows. But if $\theta$ is not so large, the net effect is ambiguous: it depends on $\kappa$, measuring how easily the bubble bursts under selling pressure. With small $\kappa$ (the case of Corollary 3.1 and Figure 4), the crash-aversion effect prevails and thus the managers sell out early, as they have a strong incentive to counterbalance the high risk of getting caught in the crash.

\subsection{Discussion}

Corollary 3.1 and Figure 4 may be surprising because they imply that, unlike what common wisdom would suggest, (1) fund managers whose compensation is highly performancesensitive may pull out of bubbles quicker than the others, and (2) fund managers' ranking concerns may help stabilize the market by eliminating bubbles quickly. Are these results 
empirically plausible? They are in line with Dass, Massa, and Patgiri (2008), who find empirical evidence that the higher the incentives in mutual fund managers' advisory contracts, the lesser these funds invest in bubble stocks. Dass, Massa, and Patgiri (2008) attribute this observation to mutual funds' herding behavior. They interpret investing in bubble stocks as herding, and not investing in them as diverging from the herd. Diverging from the herd is viewed as a high-risk-high-return strategy because it makes a fund lagging behind its peers if the bubble continues to swell, but it allows the fund to rank at the top if the bubble bursts quickly. They argue that high-incentive funds opt for such a risky strategy. Our model complements their work by offering an alternative theoretical explanation as discussed in Section 3.4: high-incentive funds may opt for staying away from bubbles as they have strong incentives to avoid outflows triggered when getting caught in the crash. There is a caveat for this argument, however, as the relation between Dass, Massa, and Patgiri (2008) and our model is not one-to-one: while they study fund managers' explicit incentives due to advisory contracts, our model is concerning implicit incentives arising from the flow-performance relationship. We are not aware of empirical works that study the relation between funds' implicit incentives and bubbles. It would be interesting for future research to study if the negative relation documented by Dass, Massa, and Patgiri (2008) applies also to implicit incentives of fund managers.

Due to the nonmonotonic relation between $\theta$ and $\left(x_{a}^{*}, x_{b}^{*}\right)$, our model also yields implications that seem to conflict with those we have just discussed. That is, if $\theta$ is large enough, ranking-concerned fund managers ride bubbles longer than those without such concerns. Interpreting the manager's type as her age or reputation in the profession, the result is consistent with the empirical finding of Greenwood and Nagel (2009) that young fund managers tend to buy bubble stocks more than old managers. Greenwood and Nagel (2009) argue that young managers tend to purchase overpriced stocks because they are inexperienced. Our result provides an additional insight: not only the managers who are actually incompetent but also those who are competent but can be potentially perceived by the market as incompetent may invest heavily in growing bubbles. Since young fund managers have little track record, it is difficult for investors to tell such a manager's skill from luck by observing only her absolute performance. Thus, investors also look at her performance relative to her peers. Then each manager is incentivized to ride bubbles long, even if she is aware of the overpricing, for fear of being perceived as an incompetent one who missed out on the benefit of swelling bubbles. Of course, riding bubbles accompanies risk of getting caught in the crash and experiencing large outflows; however, the convexity of the flow-performance relationship encourages her to take such a risk. 


\section{Extension: Heterogeneous Interim Performance}

In the model of Section 2, all managers stand at the same starting line at time 0; that is, the initial value of assets under management is the same for all of them. They set off equally and are assigned different ranks only ex post, just like in a race. However, in reality, fund managers are always on the run: their ranking is revised frequently, and thus some are ranked better than the others at any point in time. In such a situation, the managers may have risk-taking incentives that are not captured in the model of Section 2 , and the strategic interactions between these managers may affect the persistence of bubbles. Wouldn't the managers who are currently lagging behind others try to ride bubbles even longer in an attempt to make up for their inferior positions and overtake those ahead of them? Or, would they rather give up on catching up with the leaders and sell out even quicker to avoid the crash?

To answer these questions, we extend the model of Section 2 to allow for a heterogeneity in the managers' interim performance. A part of the managers are hit by a negative shock at time 0 - which we interpret as an "interim" date - and lose the value of fund assets, e.g., making a loss in other asset markets, but all managers will be evaluated under the same ranking rule eventually. If the ranking tournament is too close to call, i.e., the interim performance gap is small, then strategic interactions of the managers may lead to an even longer persistence of bubbles because the interim followers try to catch up while the interim leaders try to stay ahead. But with a large interim performance gap, bubbles may burst quickly as the interim followers give up on catching up while the interim leaders lock in their winning positions.

\subsection{Setup}

We modify the model of Section 2 as follows. All managers are ranking-concerned (i.e., $\alpha=1)$. At time 0 , an exogenous proportion $(1-\omega) \in(0,1)$ of the managers are hit by a shock that lowers the value of their assets under management from 1 to $e^{-\psi}$, where $\psi>0$. This shock is interpreted as a decline in the portfolio value caused by a loss they make in other unmodelled asset markets. The rest of the managers are intact, keeping the assets' value of 1 . Parameter $\psi$ is refer to as the interim performance gap. Those hit by the shock are referred to as type $B$, while the others are type $G$. We assume that the manager's type is independent of the time at which she becomes aware of the bubble, so that for all $t_{i} \in\left[t_{0}, t_{0}+\eta\right]$, a mass $\omega / \eta$ of type $G$ ("manager $G$-i") and $(1-\omega) / \eta$ of 
type $B$ ("manager $B$-i") become aware of the bubble. Let $x_{k, i}$ denote the holding period of manager $k-i, k \in\{G, B\}$. Irrespective of their types, all the managers are evaluated under the same ranking rule after the crash: each manager's rank is the mass of managers whose portfolio value is higher than or equal to hers.

\subsection{Manager's problem}

We look for a symmetric equilibrium in which every manager correctly believes that all managers of the same type sell out after the same holding period, i.e., $\forall i x_{k, i}=x_{k}^{*}$, $k \in\{G, B\}$.

Let us characterize each manager's rank. To simplify notation, let $\kappa_{k} \equiv\left(\tau^{*}-x_{k}^{*}\right) / \eta$, $k \in\{G, B\}$, be the proportion of type- $k$ managers who sell out before the crash in equilibrium, where $\tau^{*}$ is the endogenous duration of the bubble. ${ }^{12}$ Unlike Section 3.1, here we need to derive each type's rank separately because types $G$ and $B$ who sell out at the same time are assigned different ranks. As derived in Appendix D, the rank of manager $G$-i, which depends on her choice of $x_{G, i}$ and the stochastic crash time $t_{c}$, is

$\gamma_{G, i}\left(x_{G, i}\right)=\left\{\begin{array}{ll}\underbrace{\omega}_{\text {type } G \text { better than manager } G-i}+\underbrace{(1-\omega) \kappa_{B}^{+}}_{\text {type } B \text { better than manager } G-i} & \text { if } t_{i}+x_{G, i} \geq t_{c}, \\ {[\underbrace{\frac{\omega}{\eta} \min \left\{t_{c}-\left(t_{i}+x_{G, i}\right), \eta \kappa_{G}\right\}}_{\text {type } G \text { better than manager } G-i}+} \\ \underbrace{\max \left\{0, \frac{1-\omega}{\eta} \min \left\{t_{c}-\left(t_{i}+x_{G, i}\right)-\frac{\psi}{g}, \eta \kappa_{B}\right\}\right\}}_{\text {type } B \text { better than manager } G-i}]\end{array}\right] \begin{array}{ll}\text { if } t_{i}+x_{G, i}<t_{c},\end{array}$

where $\kappa_{B}^{+} \equiv \max \left\{0, \min \left\{\left(\tau^{*}-\psi / g\right) / \eta, \kappa_{B}\right\}\right\}$ is the proportion of type $B$ who perform better than the type- $G$ crashed managers. The first line of (4.1) corresponds to the case in which she sells out after the crash, i.e., $t_{i}+x_{G, i} \geq t_{c}$. The second line is the case in

\footnotetext{
${ }^{12}$ In other words, a mass $\omega \kappa_{G}$ of type $G$ and a mass $(1-\omega) \kappa_{B}$ of type $B$ sell out before the crash.
} 
which she sells out before the crash, i.e., $t_{i}+x_{G, i}<t_{c}$. Likewise, manager $B-i$ 's rank is

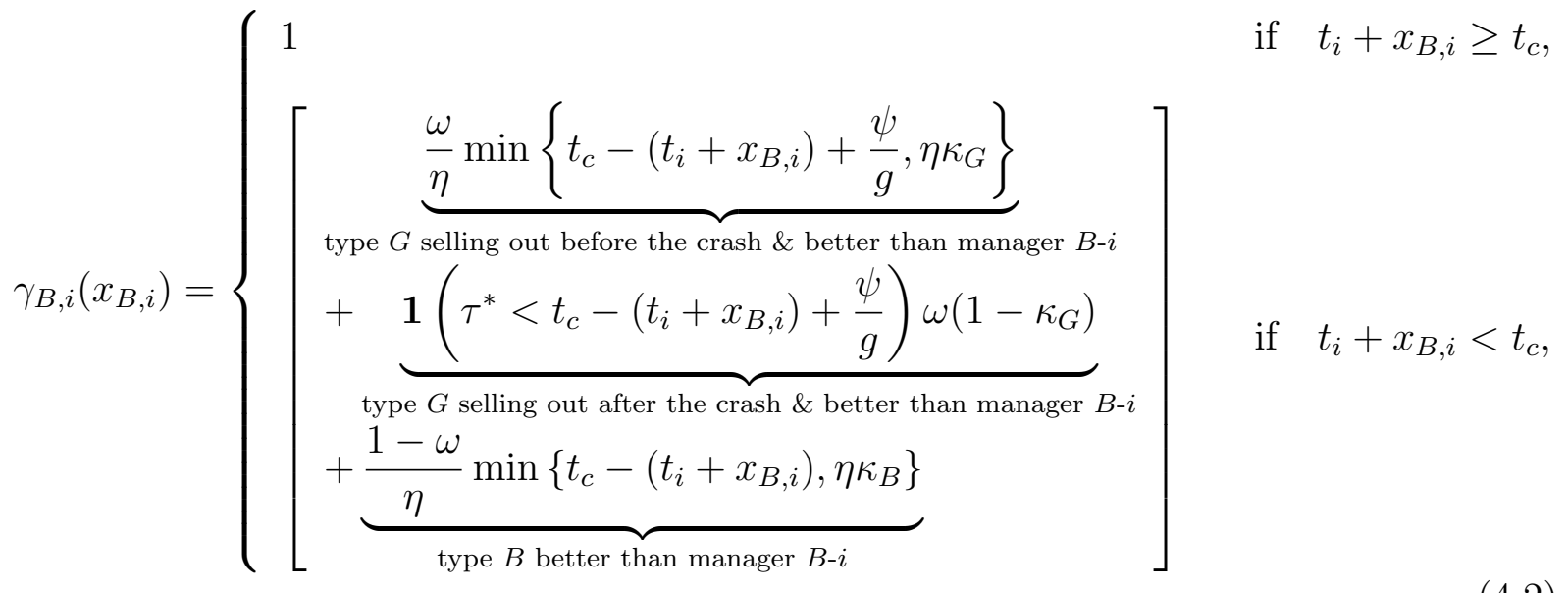

where $\mathbf{1}(Z)$ is an indicator function that takes 1 if $Z$ is true and 0 otherwise.

The problem of manager $k-i, k \in\{G, B\}$, is to choose her holding period $x_{k, i} \in[0, \infty)$, given the others' equilibrium actions $\left(x_{G}^{*}, x_{B}^{*}\right)$, to maximize the expected value of assets under management at the evaluation time. That is, her objective function is

$$
V_{k, i}\left(x_{k, i}\right)=\int_{t_{i}}^{t_{i}+x_{k, i}} e^{-\theta \gamma_{k, i}\left(x_{k, i}\right)} \widetilde{P}\left(t_{c}\right) \mathrm{d} \Pi_{i}\left(t_{c}\right)+\int_{t_{i}+x_{k, i}}^{\infty} e^{-\theta \gamma_{k, i}\left(x_{k, i}\right)} P\left(t_{i}+x_{k, i}\right) \mathrm{d} \Pi_{i}\left(t_{c}\right),
$$

where the distribution function $\Pi_{i}\left(t_{c}\right)$, common for types $G$ and $B$, is given by (3.3). ${ }^{13}$ The first term on the RHS of (4.3) corresponds to the case in which she sells out after the crash, i.e., $t_{i}+x_{k, i} \geq t_{c}$. The second term is the case in which she sells out before the crash, i.e., $t_{i}+x_{k, i}<t_{c}$.

\subsection{Equilibrium}

The symmetric equilibrium is obtained by following steps similar to those in Section 3.4. We solve each type $k$ 's FOC for the symmetric best-response function $R_{k}(\cdot)$, and identify $\left(x_{G}^{*}, x_{B}^{*}\right)$ at the intersections of $R_{G}\left(x_{B}\right)$ and $R_{B}\left(x_{G}\right)$. As in the model of Section 2 , the bubble's equilibrium duration is $\tau^{*}=\eta \kappa+\omega x_{G}^{*}+(1-\omega) x_{B}^{*}$ (see Appendix D.1). We denote by $\tilde{x}$ the benchmark holding period, which would be obtained in the model with no heterogeneity in the managers' interim performances $(\psi=0)$.

Figure 5 illustrates the determination of $\left(x_{G}^{*}, x_{B}^{*}\right)$, using different values of the interim

\footnotetext{
${ }^{13}$ The expected value of assets of each type $B$ is the entire expression of (4.3) multiplied by $e^{-\psi}$. However, since $e^{-\psi}$ is a constant, her objective function is still given by (4.3).
} 


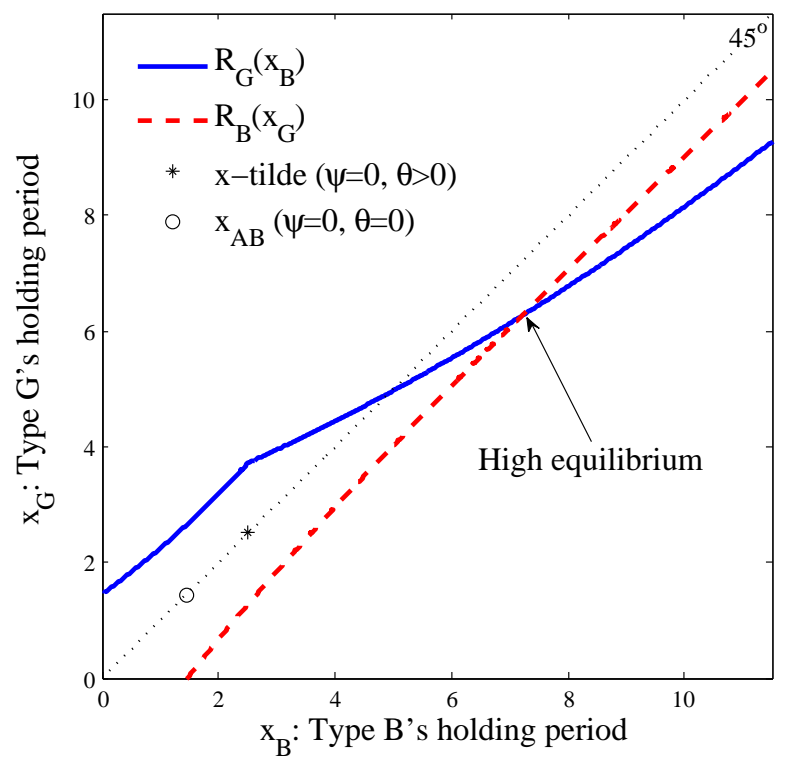

(a) Small $\psi(\psi=0.5) \Rightarrow$ high equilibrium.

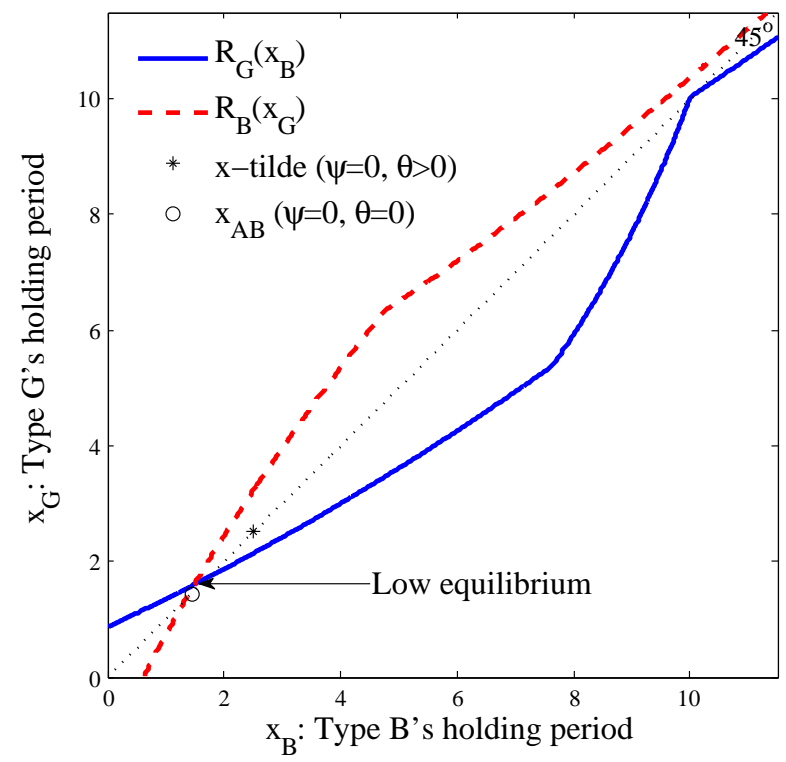

(b) Large $\psi(\psi=2) \Rightarrow$ low equilibrium.

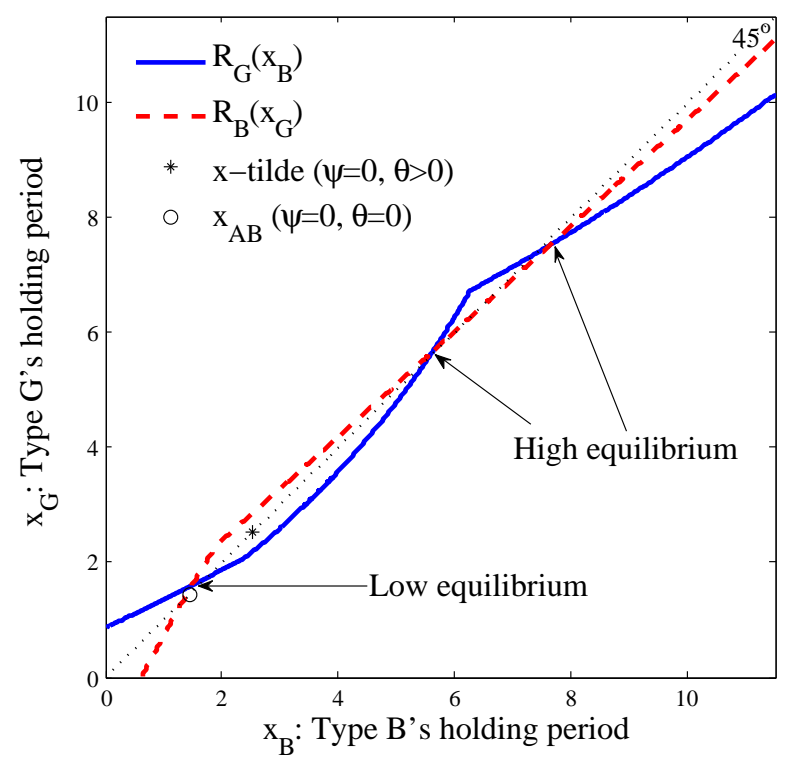

(c) Intermediate $\psi(\psi=1.25) \Rightarrow$ high \& low multiple equilibria.

Figure 5: Equilibria of the heterogeneous interim performance model. The parameter values used in the figures are $g=0.2, \lambda=0.1, \eta=7, \kappa=0.58, \omega=0.75$, and $\theta=1.3$. The benchmark holding periods are $\tilde{x}=2.5124$ and $x_{A B}=1.4438$. The equilibrium holding periods are $\left(x_{G}^{*}, x_{B}^{*}\right)=(6.2508,7.1832)$ in panel $(\mathrm{a}),\left(x_{G}^{*}, x_{B}^{*}\right)=(1.6001,1.4850)$ in panel (b), and $\left(x_{G}^{*}, x_{B}^{*}\right) \in\{(1.6001,1.4850),(5.6637,5.6176),(7.5285,7.6436)\}$ in panel (c). 
performance gap $\psi$ in three panels. In each of them, the symmetric best-response curves are upward sloping for both types and have some kinks. They are upward sloping due to the strategic complementarity between the two types: if one type rides the bubble longer it persists longer ceteris paribus, which makes it more valuable for the other type to ride it longer too as there is a lower risk of being caught in the eventual burst. The curves have kinks because the managers' risk-taking incentives represented by the slopes of $R_{k}(\cdot)$ differ with the equilibrium's "ranking type," i.e., the equilibrium classification according to "who overtakes who in the end" (see Appendix D.2 for details). ${ }^{14}$

\subsubsection{High equilibrium $\left(\left(x_{G}^{*}, x_{B}^{*}\right)>\tilde{x}\right)$ vs. low equilibrium $\left(\left(x_{G}^{*}, x_{B}^{*}\right)<\tilde{x}\right)$}

First, let us study how $x_{G}^{*}$ and $x_{B}^{*}$ depart from $\tilde{x}$ if we introduce the heterogeneity $(\psi>0)$. The implications drawn from Figure 5 are summarized as follows.

Result 4.1. If the managers' interim performances are heterogeneous, the equilibrium holding periods $\left(x_{G}^{*}, x_{B}^{*}\right)$ can be either longer or shorter than the homogeneous benchmark $\tilde{x}$, depending on the level of interim performance gap $\psi$. For small $\psi$, a high equilibrium arises: $x_{G}^{*}>\tilde{x}$ and $x_{B}^{*}>\tilde{x}$. For large $\psi$, a low equilibrium arises: $x_{G}^{*}<\tilde{x}$ and $x_{B}^{*}<\tilde{x}$. For intermediate $\psi$, we have high and low multiple equilibria.

Result 4.1 makes intuitive sense. The interim performance gap $\psi$ measures how difficult it is for each type $B$ to catch up with type $G$. First, if catching up is not so difficult, i.e., small $\psi$, then type $B$ actually try to do so by riding the bubble long. In response, type $G$ also ride the bubble long to stay ahead, since otherwise they would be easily overtaken by type $B .{ }^{15}$ Second, if catching up is difficult, i.e., large $\psi$, then type $B$ give up on catching up and sell out early. Given that, type $G$ do not need to escape

\footnotetext{
${ }^{14}$ For example, the slope of $R_{B}\left(x_{G}\right)$ at the bottom left of panel (c) differs from the one at the top right because the ranking types are different in these regions. In the potential equilibria corresponding to $\left(x_{G}, x_{B}\right)$ at the bottom-left region, even the best-performing type $B$ does not overtake the crashed type $G$, so a small change in $\left(x_{G}, x_{B}\right)$ would not cause a switch of final ranks between types. So a marginal change in $x_{G}$ has only a small impact on type $B$ 's payoffs, leading to a mild slope of $R_{B}\left(x_{G}\right)$. By contrast, in the potential equilibria corresponding to $\left(x_{G}, x_{B}\right)$ at the top-right region, both types' ranks clash with each other (i.e., there are some type $B$ whose final ranks are the same as those of type $G$ ), and thus a small change in $\left(x_{G}, x_{B}\right)$ would trigger a switch of final ranks between types. In such a case, a marginal change in $x_{G}$ has a large impact on each type $B$ 's final rank, leading her to make a large adjustment in $x_{B}$. This is why the slope of $R_{B}\left(x_{G}\right)$ is steep in this region. See Appendix D.2 for further discussions.

${ }^{15}$ Even if $\psi$ is very close to zero, $x_{G}^{*}$ and $x_{B}^{*}$ are significantly larger than $\tilde{x}$. That is, $x_{G}^{*}$ and $x_{B}^{*}$ as functions of $\psi$ are discontinuous at $\psi=0$. This is because the managers' ranks are discontinuous at $\psi=0$. Suppose that $\psi$ is positive and very close to zero. In this case, the crashed type $G$ and the crashed type $B$ perform almost identically, but the former's rank, $\omega+(1-\omega) \kappa_{B}$, is significantly better than the latter's, 1 . But if $\psi$ equals zero, they share the same rank, 1.
} 


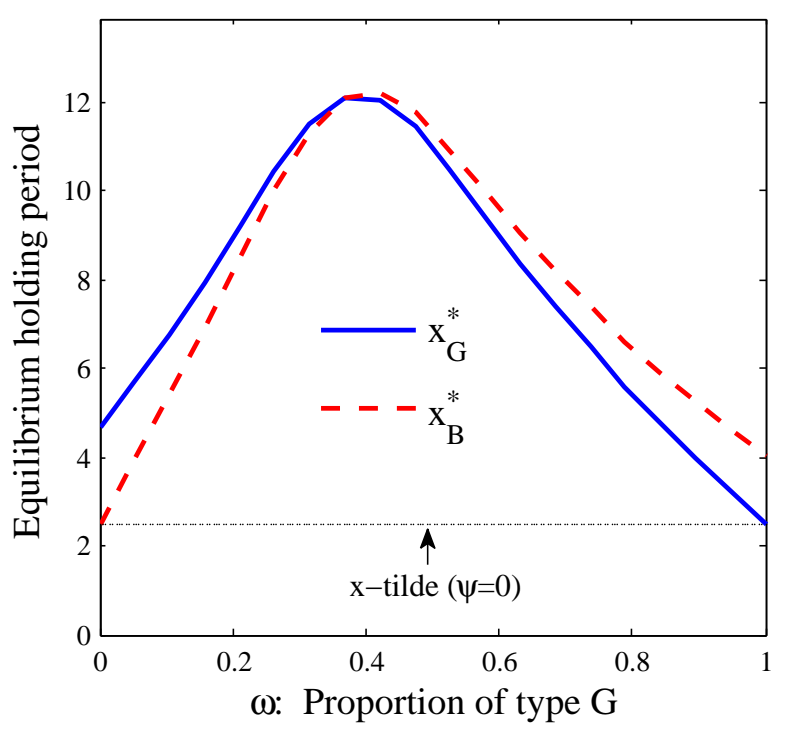

(a) High equilibrium $(\psi=0.5)$

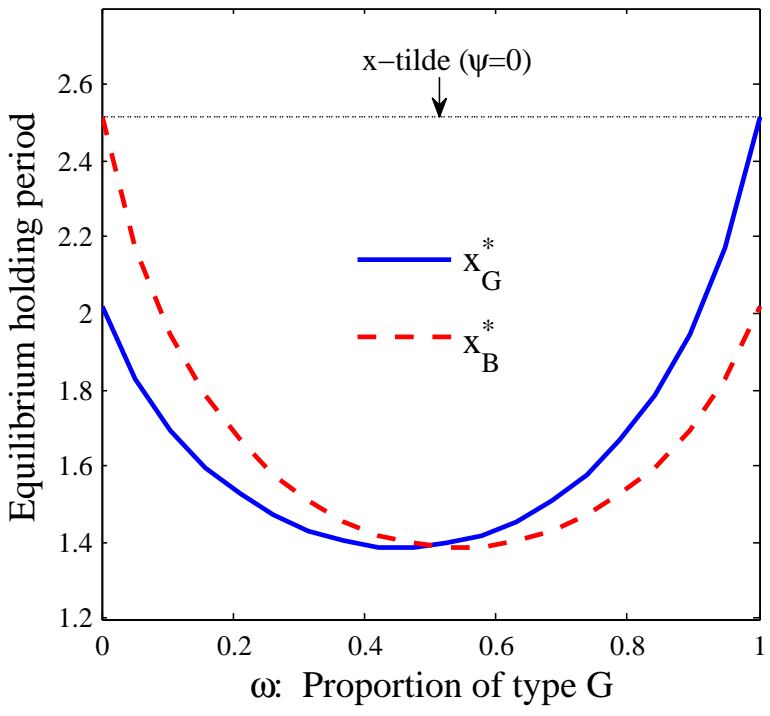

(b) Low equilibrium $(\psi=2)$

Figure 6: Equilibrium holding periods $\left(x_{G}^{*}, x_{B}^{*}\right)$ as functions of the proportion of type $G$, $\omega$. The parameter values are the same as those of Figure 5.

from type $B$; they also sell out early to lock in their winning positions. Third, if $\psi$ is in the middle range, the equilibrium can be either high or low, depending on the managers' self-fulfilling beliefs. This multiplicity is caused by the strategic complementarity between the two types of managers, as discussed already.

Since Result 4.1 is driven by the strategic interaction between types $G$ and $B$, the result is most pronounced if both of these types constitute large cohorts in the market so that their actions have significant impacts on each other's ranks. This point is illustrated in Figure 6, which plots $x_{G}^{*}$ and $x_{B}^{*}$ as functions of the proportion of type $G$, $\omega$. For intermediate levels of $\omega$, where both types of managers have large mass, the bubble persists even longer in the high equilibrium (panel (a); $\psi=0.5$ ), whereas it bursts even quicker in the low equilibrium (panel (b); $\psi=2$ ).

Result 4.1 is related to Brown, Harlow, and Starks (1996), who empirically investigate mutual fund tournaments. They find that interim-follower funds, whose performance over the first half of the year is below the industry median, tend to increase portfolio risk more than the interim-leader funds in the second half of the year. ${ }^{16}$ Consistent with their finding, our model implies that if the funds' tournament is too close to call (i.e.,

\footnotetext{
${ }^{16}$ Busse (2001) replicates the study of Brown, Harlow, and Starks (1996) with daily returns rather than monthly returns and concludes that the no-tournament hypothesis between fund managers cannot be rejected. We thank an anonymous referee for pointing this out.
} 


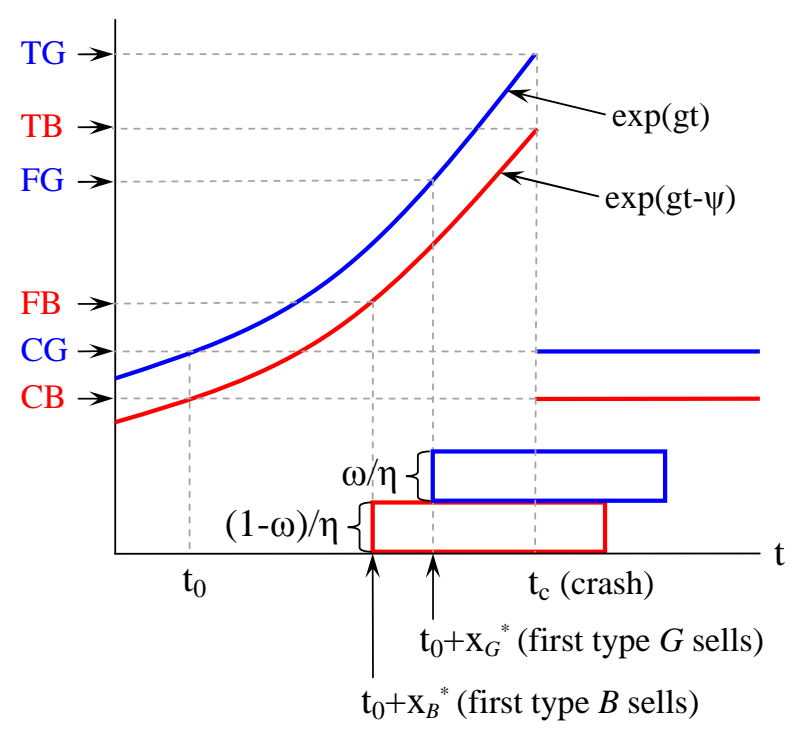

(a) $x_{G}^{*}>x_{B}^{*}$

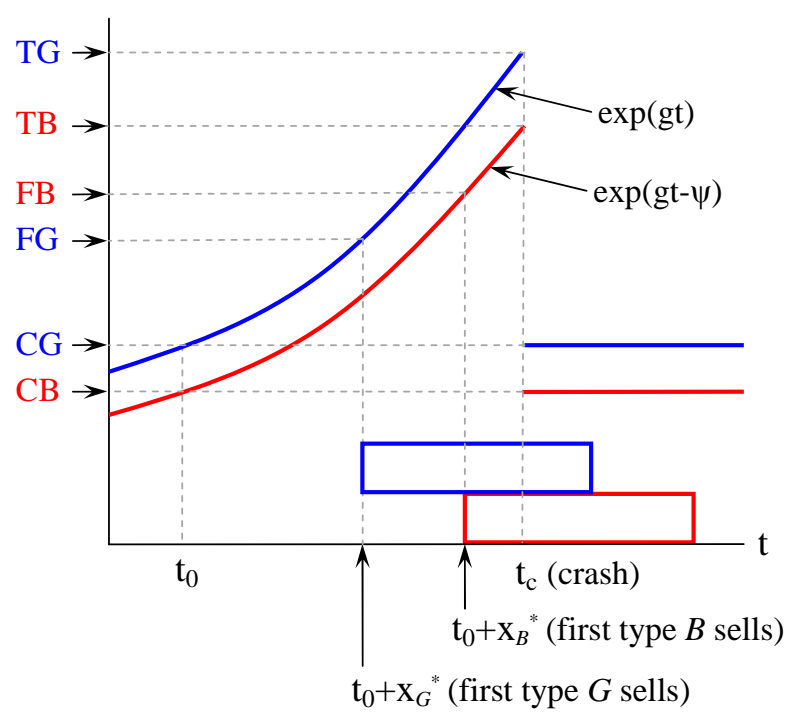

(b) $x_{B}^{*}>x_{G}^{*}$

Figure 7: Symmetric equilibrium with heterogeneous interim performance gap

$\psi$ is small), type $B$ engage in highly risky bubble riding in an attempt to make up for their low interim performance. Moreover, panel (a) of Figure 5 is in line with their result, as type $B$ takes a higher risk than type $G$ (i.e., $x_{B}^{*}>x_{G}^{*}$ ). ${ }^{17}$ However, our model also offers a different prediction: if the funds' performance gap is so large that it is already evident at an interim date which ones will be the ultimate winners and losers, the interim follower funds may give up on catching up and opt for safer strategies, i.e., selling out quickly. This prediction appears to be opposed to what Brown, Harlow, and Starks (1996) suggest. However, it is worth noting that Brown, Harlow, and Starks (1996) do not study bubble periods particularly. It will be interesting for future research to study mutual fund tournaments in bubble episodes exclusively to test our model's predictions.

\subsection{2 $x_{G}^{*}>x_{B}^{*}$ vs. $x_{B}^{*}>x_{G}^{*}$}

Figure 6 implies that both $x_{G}^{*}>x_{B}^{*}$ and $x_{B}^{*}>x_{G}^{*}$ can occur in both high and low equilibria. Here, it may sound counterintuitive that $x_{G}^{*}>x_{B}^{*}$ may occur in a high equilibrium because it appears that type $B$ can catch up with type $G$ only if they ride the bubble longer than type $G$. But such a logic does not apply to our model for the following reason. In our setting, the managers become aware of the bubble sequentially and therefore sell out

\footnotetext{
${ }^{17}$ In our model, $x_{B}^{*}>x_{G}^{*}$ is not a general result. For example, if we set $\omega$ to 0.3 or smaller, the high equilibrium in Figure 5(a) would be $x_{G}^{*}>x_{B}^{*}$, located above 45 degree line. We discuss this issue in detail in Section 4.3.2.
} 
sequentially at various price levels. Thus, even if $x_{G}^{*}>x_{B}^{*}$, some high-performing type $B$ do overtake some not-so-high-performing type $G$ and improve their ranks significantly, depending on their relative positions in the sequence. This point is shown in Figure 7, which illustrates examples of the symmetric equilibrium. Panel (a) is the equilibrium with $x_{G}^{*}>x_{B}^{*}$, and panel (b) is with $x_{B}^{*}>x_{G}^{*}$. The blue upward-sloping curve represents the price path faced by type $G, e^{g t}$, whereas the red curve is the type $B$ 's "effective price path" $e^{g t-\psi}$, which is the actual price path $e^{g t}$ adjusted downward by the interim shock $e^{-\psi}$. To see what the final ranking looks like, at the vertical axis we show the final performance levels of the "top $G$ " (i.e., the type $G$ who sells out at the highest price), the "first $G$ " (i.e., the type $G$ who sells out first), the "crashed $G$ " (i.e., the type $G$ who sell out after the crash), the top $B$, the first $B$, and the crashed $B$, which are denoted by $T G, F G, C G$, $T B, F B$, and $C B$, respectively. As seen in panel (a), despite $x_{G}^{*}>x_{B}^{*}$, type $B$ overtake type $G$ considerably. For example, since $T B>F G>C G$, the top $B$ is ranked higher than a mass $\frac{\omega}{\eta}\left(t_{c}-\frac{\psi}{g}-\left(t_{0}+x_{G}^{*}\right)\right)$ of type $G$ who sell out before the crash, as well as all the crashed type $G .{ }^{18}$ Of course, if type $B$ choose $x_{B}^{*}>x_{G}^{*}$ then they would overtake an even larger mass of type $G$ if successful (see Figure 7(b)); however, it is not necessarily optimal for them to do so as it accompanies a higher probability of getting caught in the crash. After all, whether each type $B$ plays $x_{B}^{*}<x_{G}^{*}$ or $x_{B}^{*}>x_{G}^{*}$ depends on the comparison between her benefit of increasing $x_{B}$, i.e., larger mass of type $G$ to overtake, and its cost, i.e., higher probability of being crashed. Even if she attempts to catch up with type $G$, choosing $x_{B}^{*}<x_{G}^{*}$ can be optimal for her as it allows her to overtake lowand middle-performing type $G$ yet avoid taking too much risk of being crashed.

What exactly is the determinant of $x_{G}^{*}>x_{B}^{*}$ or $x_{B}^{*}>x_{G}^{*}$ ? It is the combination of two parameters: the interim performance gap, $\psi$, and the proportion of type $G, \omega$. The implications drawn from Figure 6 are as follows.

Result 4.2. Whether $x_{G}^{*}$ is larger or smaller than $x_{B}^{*}$ depends on the combination of the interim performance gap $\psi$ and the relative population of types $G$ and $B$.

1. In the high equilibrium where $\psi$ is small, the minority type ride the bubble longer than the majority type: $x_{G}^{*}>x_{B}^{*}$ if $\omega$ is small, and $x_{G}^{*}<x_{B}^{*}$ if $\omega$ is large.

2. In the low equilibrium where $\psi$ is large, the majority type ride the bubble longer than the minority type: $x_{G}^{*}>x_{B}^{*}$ if $\omega$ is large, and $x_{G}^{*}<x_{B}^{*}$ if $\omega$ is small.

\footnotetext{
${ }^{18}$ The top $B$ sells out at time $t_{c}$ (before the crash). Hence, her performance level is equivalent to that of the type $G$ who sells out at time $t_{c}-\frac{\psi}{g}$. All the type $G$ who sell out before time $t_{c}-\frac{\psi}{g}$, constituting a mass $\frac{\omega}{\eta}\left(t_{c}-\frac{\psi}{g}-\left(t_{0}+x_{G}^{*}\right)\right)$, have lower performances than the top $B$.
} 
To see the intuition for statement 1 of Result 4.2, let us study the economics behind Figure 6(a). Because of small $\psi$, it is not difficult for type $B$ to catch up with type $G$; so both $x_{G}^{*}$ and $x_{B}^{*}$ are greater than $\tilde{x}$ for all $\omega$, as type $B$ try catching up while type $G$ try to stay ahead (Result 4.1). How much $x_{G}^{*}$ and $x_{B}^{*}$ deviate from $\tilde{x}$ depends on $\omega$. Specifically, the minority type's action - whether it is $G$ or $B$-deviates more. The intuition is the following. First, suppose $\omega$ is very small, i.e., type $B$ constitute the vast majority of the population. In this case, type $G$ 's actions have only a small impact on type $B$ 's ranks, so type $B$ nearly neglect the presence of type $G$; thus, type $B$ 's action is close to the homogeneous case (i.e., $x_{B}^{*} \rightarrow \tilde{x}$ as $\omega \rightarrow 0$ ). Small $\psi$ induces each type $G$ to choose relatively large $x_{G}^{*}$ to stay ahead, since otherwise she could be easily overtaken by type $B$ who are close behind. ${ }^{19}$ Her incentive to stay ahead becomes stronger as $\omega$ gets closer to 0 , because her rank would fall sharply if she is overtaken by a large mass of type $B$. In contrast, type $B$ 's incentive to catch up with type $G$ becomes weaker as $\omega$ gets closer to 0 , since their benefit from overtaking type $G$ decreases as the mass of type $G$ decreases. Consequently, $x_{G}^{*}>x_{B}^{*}$ holds for small $\omega$ and the distance between $x_{G}^{*}$ and $x_{B}^{*}$ widens as $\omega$ gets closer to 0 . Second, suppose $\omega$ is very large, i.e., the vast majority is type $G$. In this case, type $G$ almost neglect type $B$ and behave as in the homogeneous case (i.e., $x_{G}^{*} \rightarrow \tilde{x}$ as $\left.\omega \rightarrow 1\right)$. Since each type $B$ is lagging behind a large mass of managers at the interim date, large outflows will be inevitable unless she rides the bubble long to make up for her poor interim performance. So she chooses large $x_{B}^{*}$ as a gamble for resurrection, leading to $x_{B}^{*}>x_{G}^{*} \cdot{ }^{20}$

The intuition for statement 2 of Result 4.2 is drawn from Figure 6(b). Due to large $\psi$, catching up is difficult for type $B$; so both $x_{G}^{*}$ and $x_{B}^{*}$ are smaller than $\tilde{x}$, as type $B$ give up on catching up while type $G$ lock in their winning positions (Result 4.1). In such a case, the minority type has a strong incentive to sell out early - that is, the majority type ride the bubble relatively long - for the following reason. First, suppose $\omega$ is very large. In this case, type $G$ act as in the homogeneous case (i.e., $x_{G}^{*} \rightarrow \tilde{x}$ as $\omega \rightarrow 1$ ). Since each type $B$ is lagging behind most managers at the interim date and yet $\psi$ is so large that she has little chance of catching up even if she rides the bubble long, the best she can hope for is to avoid getting caught in the crash and secure a middle rank. Thus, she

\footnotetext{
${ }^{19}$ Note that, in this particular situation where $\psi$ is small, type $G$ would not try to lock in their winning positions by selling out early because it would put themselves at high risk of being overtaken at a stroke by type $B$ who successfully ride the bubble.

${ }^{20}$ Moreover, the fact that the gain from bubble-riding (if successful) is large encourages her gambling: since the mass of type $G$ is much larger than that of type $B$, if successful, each type $B$ can overtake a large mass of type $G$ at a stroke.
} 
chooses small $x_{B}^{*}$ in a bid to pull out quicker than her peers, leading to $x_{G}^{*}>x_{B}^{*}$. Second, suppose $\omega$ is very small. In this case, type $B$ behave as in the homogeneous case (i.e., $x_{B}^{*} \rightarrow \tilde{x}$ as $\left.\omega \rightarrow 0\right)$. The combination of large $\psi$ and small $\omega$ means that each type $G$ is in a very strong position at the interim date, as most managers are way behind her and unlikely to catch up. That is, she is guaranteed a high rank unless being crashed. So she chooses a small $x_{G}^{*}$ to lock in her winning position, leading to $x_{B}^{*}>x_{G}^{*}$.

A corollary of Result 4.2 is that, despite heterogeneity in interim performances, all managers could choose the same action $\left(x_{G}^{*}=x_{B}^{*}\right)$ for an intermediate level of $\omega$. Indeed, in both Figures $6(\mathrm{a})$ and $6(\mathrm{~b})$, the curves of $x_{G}^{*}$ and $x_{B}^{*}$ cross. The intuition for the highequilibrium case is as follows. We have $x_{G}^{*}>x_{B}^{*}$ for $\omega \approx 0$, as type $G$ 's stay-ahead motive dominates type $B$ 's catch-up motive. We have $x_{B}^{*}>x_{G}^{*}$ for $\omega \approx 1$, as type $B$ 's catch-up motive dominates type $G$ 's stay-ahead motive. But $x_{G}^{*}$ and $x_{B}^{*}$ are close to each other for the middle range of $\omega$ since both of the motives are at similar levels. At a critical level of $\omega$ for which these two motives are exactly equilibrated, we obtain $x_{G}^{*}=x_{B}^{*}$. The intuition for the low-equilibrium case is similar.

That $x_{B}^{*}>x_{G}^{*}$ may hold in a high equilibrium is in line with the existing models with relative performance objectives, such as Hvide (2002), Cabral (2003), or Goriaev, Palomino, and Prat (2003), in that interim laggards use a riskier strategy than interim leaders. However, unlike these models, ours predicts that $x_{G}^{*}>x_{B}^{*}$ may also occur. There are two main differences between the existing models and ours that allow $x_{G}^{*}>x_{B}^{*}$ to arise. First, as noted at the beginning of this section, our model features partial catchup: high-performing type $B$ catch up with low- and middle-performing type $G$, though top-performing type $G$ are not overtaken by anyone. Due to this setting, $x_{B}^{*}<x_{G}^{*}$ can be consistent with each type $B$ 's incentive to catch up with type $G$, as it still allows her to overtake not-so-high-performing type $G$. By contrast, in the papers cited above, the interim laggards need to completely overtake the interim leaders to benefit from their catching-up strategy. For example, in Goriaev, Palomino, and Prat (2003), which is most closely related to ours, the interim laggard either successfully overtakes the interim leader and obtains a bonus or fails to overtake and misses that bonus. Thus, the laggard is compelled to take a higher risk than the leader to overturn the interim ranking. Second, in our model, the relative population of types $G$ and $B$ plays a key role in the equilibrium characterization. Indeed, as discussed in Result 4.2, a high equilibrium with $x_{G}^{*}>x_{B}^{*}$ arises only if the presence of type $G$ is small, i.e., $\omega$ is small, and therefore type $B$ do not have a strong incentive to increase $x_{B}^{*}$ to catch up with type $G$ who choose large $x_{G}^{*}$. Such a case is not analyzed in the models cited above. For instance, the benchmark model of 
Goriaev, Palomino, and Prat (2003) considers two fund managers with the same size. It corresponds to the case of $\omega=1 / 2$ in our model. Indeed, for $\omega=1 / 2$ our model also yields $x_{B}^{*}>x_{G}^{*}$ in the high equilibrium (see Figure $6(\mathrm{a})$ ), consistent with their result.

\section{Robustness: Random Evaluation Time}

So far, we have assumed that the managers' performance evaluation occurs at some point after the crash. In this section, we consider an alternative setting in which the evaluation takes place stochastically, either before or after the crash. This can be interpreted as the authority's random inspection of funds' balance sheets. We show that the key result of the model of Section 2 that the manager's holding period and the associated duration of the bubble are U-shaped in the flow-performance sensitivity $\theta$ is robust to such a modification.

For simplicity, we assume that all the managers are ranking-concerned, i.e., $\alpha=1$ in Section 2. The manager who becomes aware of the bubble at time $t_{i}$ (manager $i$ ) sells out at time $t_{i}+x_{i}$. We denote by $x^{* *}$ the symmetric equilibrium holding period. The evaluation time, $t_{e}$, is distributed on $[0, \infty)$ with the cumulative distribution function $H\left(t_{e}\right)=1-e^{-\phi t_{e}}$, where $\phi>0$ is the rate parameter. At time $t_{e}$, each manager $i$ is assigned a rank $\gamma_{i}\left(x_{i}\right)$, which is the mass of managers whose investment returns from time 0 to $t_{e}$ are higher or equal to hers.

Manager $i$ 's rank depends on the relative position of her sell out time $\left(t_{i}+x_{i}\right)$ to the crash time $\left(t_{c}\right)$, the first manager's sell out time $\left(t_{0}+x^{* *}\right)$, and the evaluation time $\left(t_{e}\right)$ as follows.

- If $t_{i}+x_{i} \geq t_{c}$

$$
\gamma_{i}\left(x_{i}\right)= \begin{cases}1 & \text { for } t_{e} \leq t_{0}+x^{* *} \\ 1-\frac{1}{\eta}\left(t_{e}-\left(t_{0}+x^{* *}\right)\right) & \text { for } t_{e} \in\left(t_{0}+x^{* *}, t_{c}\right), \\ 1 & \text { for } t_{e} \geq t_{c}\end{cases}
$$

- If $t_{i}+x_{i} \in\left(t_{0}+x^{* *}, t_{c}\right)$,

$$
\gamma_{i}\left(x_{i}\right)= \begin{cases}1 & \text { for } t_{e} \leq t_{0}+x^{* *} \\ 1-\frac{1}{\eta}\left(t_{e}-\left(t_{0}+x^{* *}\right)\right) & \text { for } t_{e} \in\left(t_{0}+x^{* *}, t_{i}+x_{i}\right) \\ 1-\frac{1}{\eta}\left(\left(t_{i}+x_{i}\right)-\left(t_{0}+x^{* *}\right)\right) & \text { for } t_{e} \in\left(t_{i}+x_{i}, t_{c}\right) \\ \frac{1}{\eta}\left(t_{c}-\left(t_{i}+x_{i}\right)\right) & \text { for } t_{e} \geq t_{c}\end{cases}
$$


- If $t_{i}+x_{i} \leq t_{0}+x^{* *}$,

$$
\gamma_{i}\left(x_{i}\right)= \begin{cases}1 & \text { for } t_{e}<t_{c} \\ \kappa & \text { for } t_{e} \geq t_{c} .\end{cases}
$$

(5.1) is the manager's rank when she sells out after the crash; (5.2) corresponds to the case in which she sells out before the crash and after some other managers; (5.3) is the off-equilibrium case in which she sells out before any other managers.

Manager $i$ 's problem at time $t_{i}$ is to choose her holding period $x_{i} \in[0, \infty)$, given that the other managers choose the equilibrium holding period $x^{*}$, to maximize the expected value of assets under management as of time $t_{e}$ :

$$
\begin{aligned}
V_{i}\left(x_{i}\right) & =\int_{t_{i}}^{t_{i}+x_{i}}\left\{\begin{array}{c}
\int_{0}^{t_{c}} e^{-\theta \gamma_{i}\left(x_{i}\right)} P\left(t_{e}\right) \mathrm{d} H\left(t_{e}\right) \\
+\int_{t_{c}}^{\infty} e^{-\theta \gamma_{i}\left(x_{i}\right)} \widetilde{P}\left(t_{c}\right) \mathrm{d} H\left(t_{e}\right)
\end{array}\right\} \mathrm{d} \Pi_{i}\left(t_{c}\right) \\
& +\int_{t_{i}+x_{i}}^{\infty}\left\{\begin{array}{c}
\int_{0}^{t_{i}+x_{i}} e^{-\theta \gamma_{i}\left(x_{i}\right)} P\left(t_{e}\right) \mathrm{d} H\left(t_{e}\right) \\
+\int_{t_{i}+x_{i}}^{\infty} e^{-\theta \gamma_{i}\left(x_{i}\right)} P\left(t_{i}+x_{i}\right) \mathrm{d} H\left(t_{e}\right)
\end{array}\right\} \mathrm{d} \Pi_{i}\left(t_{c}\right) .
\end{aligned}
$$

The first line of (5.4) corresponds to the case in which the crash occurs before she sells out $\left(t_{c} \leq t_{i}+x_{i}\right)$. In this case, if the evaluation occurs before the crash $\left(t_{e}<t_{c}\right)$, the manager's performance is based on the bubble price at the evaluation time, $P\left(t_{e}\right)$; but if it occurs after the crash $\left(t_{e} \geq t_{c}\right)$, her performance is the post-crash price, $\widetilde{P}\left(t_{c}\right)$. The second line of (5.4) corresponds to the case in which she sells out before the crash $\left(t_{i}+x_{i}<t_{c}\right)$. In this case, if the evaluation is before she sells out $\left(t_{e}<t_{i}+x_{i}\right)$, her performance is based on the bubble price at the evaluation time, $P\left(t_{e}\right)$; but if it is after she sells out $\left(t_{e} \geq t_{i}+x_{i}\right)$, her performance is her sell-out price, $P\left(t_{i}+x_{i}\right)$.

The equilibrium holding period $x^{* *}$ is obtained by evaluating the manager's FOC, $\mathrm{d} V_{i}\left(x_{i}\right) / \mathrm{d} x_{i}=0$, at $x_{i}=x^{* *}$ (see Appendix E).

Proposition 5.1. If the performance evaluation takes place at time te that follows c.d.f. $H\left(t_{e}\right)=1-e^{-\phi t_{e}}$, the symmetric equilibrium holding period is

$$
x^{* *}=-\frac{1}{g} \ln K-\eta \kappa,
$$




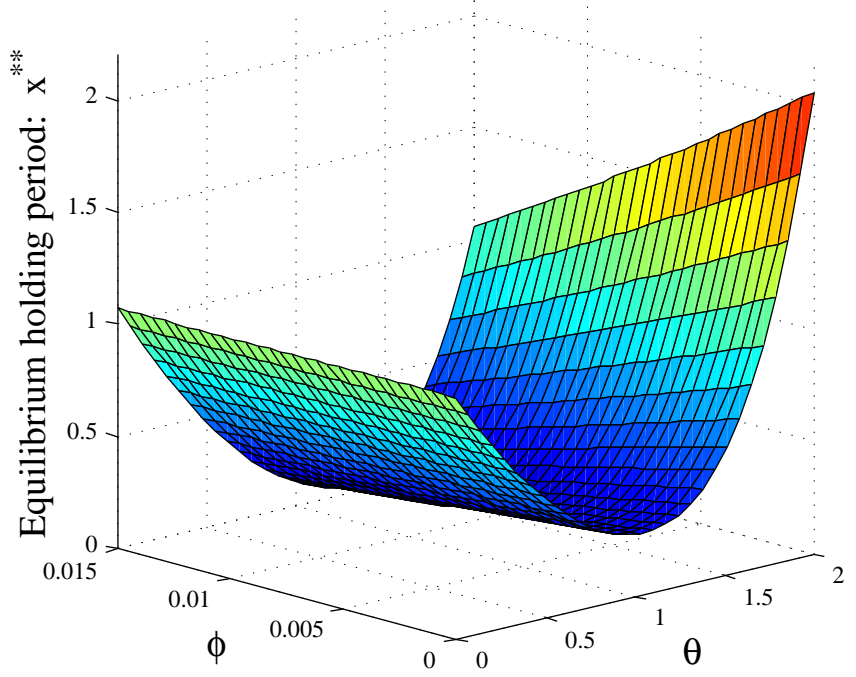

Figure 8: Random evaluation model. The figure plots the symmetric equilibrium holding period $x^{* *}$ as a function of $(\theta, \phi)$. The parameter values used in the figure are $g=0.2$, $\lambda=0.05, \eta=6$, and $\kappa=0.5$.

$$
\text { where } \begin{aligned}
K & \equiv\left(\frac{g}{g-\phi} e^{-\lambda \eta \kappa}-e^{-(\lambda+\phi) \eta \kappa}\right)-\phi\left(\frac{e^{\theta \kappa}-e^{-\lambda \eta \kappa}}{g-\phi+\frac{\theta}{\eta}}+\frac{e^{-\lambda \eta \kappa}}{g-\phi}\right) \\
& -e^{\theta \kappa}(g-\phi-\lambda)\left(\frac{g+\frac{\theta}{\eta}}{g-\phi+\frac{\theta}{\eta}} \Lambda\left(0, \eta \kappa, \frac{\theta}{\eta}\right)-\Lambda\left(0, \eta \kappa, \phi+\frac{\theta}{\eta}\right)\right) \\
& +e^{\theta}\left(1-\left(g+\frac{\theta}{\eta}\right) \Lambda\left(0, \eta \kappa, \phi+\frac{\theta}{\eta}\right)\right) .
\end{aligned}
$$

Proposition 5.1 provides a closed form solution for $x^{* *}$. Figure 8 plots $x^{* *}$ as a function of the flow-performance sensitivity $\theta$ and the rate parameter $\phi$ of $H\left(t_{e}\right)$. As in the model of Section $2, x^{* *}$ and the bubble's duration $\tau^{* *}=\eta \kappa+x^{* *}$ are U-shaped in $\theta$.

Corollary 5.1. In the limit of $\phi \rightarrow 0$, the solution of Proposition 5.1 converges to that of Proposition 3.1, i.e., $\lim _{\phi \rightarrow 0} x^{* *}=\left.x_{a}^{*}\right|_{\alpha=1}$.

The intuition for Corollary 5.1 is simple. As $\phi$ decreases, it becomes more likely that the evaluation takes place after the crash. In the limit of $\phi \rightarrow 0$, the evaluation occurs after the crash with probability one. The solution in such a case coincides with the one of Section 2 where the evaluation is after the crash by assumption. It is easy to check this result, as the first two lines of (5.6) converge to zero as $\phi \rightarrow 0$. 


\section{Conclusion}

This paper studies the implications of fund managers' relative-performance concerns for their investment behavior in bubble periods and the associated persistence of bubbles. We consider a model à la Abreu and Brunnermeier (2003), in which the managers' payoffs are sensitive to their performance ranking through the documented convex flow-performance relationship. The model implies that ranking-concerned fund managers have nonmonotonic impacts on bubbles: they may either magnify or dampen bubbles, depending on how much of a winner-take-all situation they are in. If they engage in a severe ranking tournament with highly convex flow-performance relationship, they fuel bubbles by riding them long (the risk-shifting effect). However, with moderate convexity of the relationship, they help dampen bubbles by attacking them quickly (the crash-aversion effect). These results provide theoretical explanations to the observed cross-sectional variation in funds' investment strategies in bubble periods (Dass, Massa, and Patgiri 2008; Greenwood and Nagel 2009). Heterogeneity in the managers' interim performance affects the persistence of bubbles significantly. If the tournament is too close to call (i.e., the interim performance gap is small), it induces even longer bubble-riding, as the interim followers try to catch up while the interim leaders try to stay ahead. But with a large interim performance gap, bubbles may burst quickly, as the interim followers give up on catching up while the interim leaders lock in their winning positions.

It would be a fruitful future research to explore the roles of ranking concerns in the emergence (as opposed to the persistence) of bubbles, which we take as exogenous in this

paper. A good starting point may be the model of Doblas-Madrid (2012), who extends the framework of $\mathrm{AB}$ by endogeneizing the growing bubble price. Exploring the implications of relative performance evaluations for the entire cycle of bubbles (emergence, persistence, and burst) is interesting and may yield valuable policy implications. 


\section{Appendix}

\section{A Formulas}

Let $x_{k}^{*}$ be the symmetric-equilibrium holding period of type $k$, and $\tau^{*}$ be the bubble's equilibrium duration. Let $\Lambda$ be a function defined in (3.14), and $X, Y$, and $Z$ be constants. Then we have the following results, which are all obtained by straightforward algebra.

$$
\begin{gathered}
\int_{t_{i}+x_{k, i}+X}^{t_{i}+x_{k, i}+Y} e^{-Z\left(t_{c}-\left(t_{i}+x_{k, i}\right)\right)} \pi_{i}\left(t_{c}\right) \mathrm{d} t_{c}=\pi_{i}\left(t_{i}+x_{k, i}\right) \Lambda(X, Y, Z) . \\
\left.\frac{\mathrm{d}\left(\int_{t_{i}}^{t+x_{k, i}} e^{-Z\left(t_{c}-\left(t_{i}+x_{k, i}\right)\right)} P\left(t_{i}+x_{k, i}\right) \pi_{i}\left(t_{c}\right) \mathrm{d} t_{c}\right)}{\mathrm{d} x_{k, i}}\right|_{x_{k, i}=x_{k}^{*}} \\
=P\left(t_{i}+x_{k}^{*}\right) \pi_{i}\left(t_{i}+x_{k}^{*}\right)\left(1+(g+Z) \Lambda\left(-x_{k}^{*}, 0, Z\right)\right) .
\end{gathered}
$$

If $0 \leq X \leq \tau^{*}-x_{k}^{*}$ and $0 \leq Y \leq \tau^{*}-x_{k}^{*}$, then

$$
\begin{aligned}
& \left.\frac{\mathrm{d}\left(\int_{t_{i}+x_{k, i}+X}^{t_{i}+x_{k, i}+Y} e^{-Z\left(t_{c}-\left(t_{i}+x_{k, i}\right)\right)} P\left(t_{i}+x_{k, i}\right) \pi_{i}\left(t_{c}\right) \mathrm{d} t_{c}\right)}{\mathrm{d} x_{k, i}}\right|_{x_{k, i}=x_{k}^{*}} \\
& =P\left(t_{i}+x_{k}^{*}\right) \pi_{i}\left(t_{i}+x_{k}^{*}\right)(g-\lambda) \Lambda(X, Y, Z) .
\end{aligned}
$$

If $0 \leq X \leq \tau^{*}-x_{k}^{*}$, then

$$
\begin{aligned}
& \left.\frac{\mathrm{d}\left(\int_{t_{i}+x_{k, i}+X}^{\infty} e^{-Z\left(t_{c}-\left(t_{i}+x_{k, i}\right)\right)} P\left(t_{i}+x_{k, i}\right) \pi_{i}\left(t_{c}\right) \mathrm{d} t_{c}\right)}{\mathrm{d} x_{k, i}}\right|_{x_{k, i}=x_{k}^{*}} \\
& =P\left(t_{i}+x_{k}^{*}\right) \pi_{i}\left(t_{i}+x_{k}^{*}\right)\left((g+Z) \Lambda\left(X, \tau^{*}-x_{k}^{*}, Z\right)-e^{-(\lambda+Z) X}\right) .
\end{aligned}
$$

\section{B Proof of Proposition 3.1}

\section{B.1 Duration of the bubble}

If the equilibrium holding periods are $\left(x_{a}^{*}, x_{b}^{*}\right)$, the duration $\tau^{*}$ of the bubble is determined as follows.

Lemma B.1. In the symmetric equilibrium, the crash occurs at time $t_{c}=t_{0}+\tau^{*}$, where

$$
\tau^{*}=\eta \kappa+\alpha x_{a}^{*}+(1-\alpha) x_{b}^{*}
$$

\section{Proof of Lemma B.1.}

In the symmetric equilibrium, there must be both type $a$ and type $b$ who sell out at the crash time $t_{c}=t_{0}+\tau^{*}$. The reason is as follows. It cannot be an equilibrium that all type- $k$ managers $(k \in\{a, b\})$ 
sell out before the crash, because each of them would be better off by increasing $x_{k, i}$ by a small amount without any risk of getting caught in the crash. Likewise, it cannot be an equilibrium that all type- $k$ managers sell out after the crash, because each of them would obtain a chance to sell out before the crash by decreasing $x_{k, i}$. Therefore, there must be both type $a$ and type $b$ who sell out at the crash time. Since type $a$ start selling out at time $t_{0}+x_{a}^{*}$ and the crash occurs at time $t_{0}+\tau^{*}$, the mass of type $a$ who sell out before the crash is $\left(\left(t_{0}+\tau^{*}\right)-\left(t_{0}+x_{a}^{*}\right)\right) \alpha / \eta=\alpha\left(\tau^{*}-x_{a}^{*}\right) / \eta$. Similarly, the mass of type $b$ selling out before the crash is $\left(\left(t_{0}+\tau^{*}\right)-\left(t_{0}+x_{b}^{*}\right)\right)(1-\alpha) / \eta=(1-\alpha)\left(\tau^{*}-x_{b}^{*}\right) / \eta$. Since a mass $\kappa$ of managers sell out before the crash, we have $\alpha\left(\tau^{*}-x_{a}^{*}\right) / \eta+(1-\alpha)\left(\tau^{*}-x_{b}^{*}\right) / \eta=\kappa$. Solving this for $\tau^{*}$ yields (B.1). (End of proof of Lemma B.1.)

\section{B.2 Manager's rank}

Lemma B.2. Given that other managers choose $\left(x_{a}, x_{b}\right)$ and the crash occurs at time $t_{c}=t_{0}+\tau$, manager $k$-i's rank (3.1) is rewritten as

$$
\gamma_{k, i}=\gamma_{i}\left(x_{k, i}\right)= \begin{cases}1 & \text { if } t_{c} \leq t_{i}+x_{k, i} \\ \frac{1}{\eta}\left(t_{c}-\left(t_{i}+x_{k, i}\right)\right) & \text { if } t_{i}+x_{k, i}<t_{c} \leq t_{i}+x_{k, i}+\rho_{1}, \\ \varphi_{1}+\varphi_{2}\left(t_{c}-\left(t_{i}+x_{k, i}\right)\right) & \text { if } t_{i}+x_{k, i}+\rho_{1}<t_{c} \leq t_{i}+x_{k, i}+\rho_{2}, \\ \kappa & \text { if } t_{i}+x_{k, i}+\rho_{2}<t_{c}\end{cases}
$$

where $\rho_{1} \equiv \tau-\max \left\{x_{a}, x_{b}\right\}, \rho_{2} \equiv \tau-\min \left\{x_{a}, x_{b}\right\}$

$$
\varphi_{1} \equiv \mathbf{1}\left(x_{a} \geq x_{b}\right) \alpha \kappa_{a}+\mathbf{1}\left(x_{b}>x_{a}\right)(1-\alpha) \kappa_{b}, \text { and } \varphi_{2} \equiv \mathbf{1}\left(x_{a} \geq x_{b}\right) \frac{1-\alpha}{\eta}+\mathbf{1}\left(x_{b}>x_{a}\right) \frac{\alpha}{\eta} .
$$

\section{Proof of Lemma B.2.}

Step 1. Suppose $x_{a} \geq x_{b}$. There are four cases to consider. The first case is that the manager sells out after the crash $\left(t_{i}+x_{k, i} \geq t_{c}\right)$. Clearly, her rank in this case is 1 . The second case is that she sells out before the crash and after the first type $a$ sells out $\left(t_{i}+x_{k, i}<t_{c}\right.$ and $\left.t_{i}+x_{k, i} \geq t_{c}-\left(\tau-x_{a}\right)\right)$. Type $b$ have already started selling out because $x_{a} \geq x_{b}$. In this case, the mass of type $a$ performing better than her is $(\alpha / \eta)\left(t_{c}-\left(t_{i}+x_{k, i}\right)\right)$, and that of type $b$ is $((1-\alpha) / \eta)\left(t_{c}-\left(t_{i}+x_{k, i}\right)\right)$. Her rank is the sum of these two: $\left(t_{c}-\left(t_{i}+x_{k, i}\right)\right) / \eta$. The third case is that she sells out before the first type $a$ and after the first type $b\left(t_{i}+x_{k, i}<t_{c}-\left(\tau-x_{a}\right)\right.$ and $\left.t_{i}+x_{k, i} \geq t_{c}-\left(\tau-x_{b}\right)\right)$. In this case, the mass of type $a$ performing better than her (i.e., all type $a$ selling out before the crash) is $\alpha \kappa_{a}$, and that of type $b$ is $((1-\alpha) / \eta)\left(t_{c}-\left(t_{i}+x_{k, i}\right)\right)$. Her rank is the sum of these two. The fourth is the off-equilibrium case, in which she sells out even before the first type $b\left(t_{i}+x_{k, i}<t_{c}-\left(\tau-x_{b}\right)\right)$. In this case, all the managers selling out before the crash will perform better than her, that is, her rank is $\kappa$.

Step 2. Suppose $x_{b}>x_{a}$. The manager's rank in this case is obtained by replacing $x_{a}, x_{b}$, and $\alpha$ in step 1 with $x_{b}, x_{a}$, and $(1-\alpha)$, respectively.

Combining the rank obtained in steps 1 and 2 into one, we get (B.2). (End of proof of Lemma B.2.) 


\section{B.3 Manager's optimization}

Using (B.2), manager $a-i$ 's objective function (3.4) is

$$
\begin{aligned}
V_{a, i}\left(x_{a, i}\right)= & \int_{t_{i}}^{t_{i}+x_{a, i}} e^{-\theta} \widetilde{P}\left(t_{c}\right) \pi_{i}\left(t_{c}\right) \mathrm{d} t_{c} \\
& +\int_{t_{i}+x_{a, i}}^{t_{i}+x_{a, i}+\rho_{1}} e^{-\frac{\theta}{\eta}\left(t_{c}-\left(t_{i}+x_{a, i}\right)\right)} P\left(t_{i}+x_{a, i}\right) \pi_{i}\left(t_{c}\right) \mathrm{d} t_{c} \\
& +\int_{t_{i}+x_{a, i}+\rho_{1}}^{t_{i}+x_{a, i}+\rho_{2}} e^{-\theta\left(\varphi_{1}+\varphi_{2}\left(t_{c}-\left(t_{i}+x_{a, i}\right)\right)\right)} P\left(t_{i}+x_{a, i}\right) \pi_{i}\left(t_{c}\right) \mathrm{d} t_{c} \\
& +\int_{t_{i}+x_{a, i}+\rho_{2}}^{\infty} e^{-\theta \kappa} P\left(t_{i}+x_{a, i}\right) \pi_{i}\left(t_{c}\right) \mathrm{d} t_{c} .
\end{aligned}
$$

Manager $b$-i's objective function $V_{b, i}\left(x_{b, i}\right)$ is obtained by setting $\theta=0$ and replacing $x_{a, i}$ with $x_{b, i}$ in (B.3). For $k \in\{a, b\}$, manager $k-i$ 's FOC is $\mathrm{d} V_{k, i}\left(x_{k, i}\right) / \mathrm{d} x_{k, i}=0$.

\section{B.4 Equilibrium conditions}

In the symmetric equilibrium, we have $x_{k, i}=x_{k}^{*}$ for all $i$ and $k \in\{a, b\}$. Thus, evaluating the FOCs at $x_{k, i}=x_{k}^{*}$, we have

$$
\begin{aligned}
& \left.\frac{\mathrm{d} V_{a, i}\left(x_{a, i}\right)}{\mathrm{d} x_{a, i}}\right|_{x_{a, i}=x_{a}^{*}}=0, \\
& \left.\frac{\mathrm{d} V_{b, i}\left(x_{b, i}\right)}{\mathrm{d} x_{b, i}}\right|_{x_{b, i}=x_{b}^{*}}=0 .
\end{aligned}
$$

In order to obtain a parametric expression for (B.4), rearrange (B.3) so that we can apply the formulas of Appendix A.

$$
\begin{aligned}
& V_{a, i}\left(x_{a, i}\right)=e^{-\theta-g \tau^{*}} \underbrace{\int_{t_{i}}^{t_{i}+x_{a, i}} e^{g\left(t_{c}-\left(t_{i}+x_{a, i}\right)\right)} P\left(t_{i}+x_{a, i}\right) \pi_{i}\left(t_{c}\right) \mathrm{d} t_{c}}_{\text {Use (A.2) with } Z=-g} \\
& +\underbrace{\int_{t_{i}+x_{a, i}}^{t_{i}+x_{a, i}+\rho_{1}} e^{-\frac{\theta}{\eta}\left(t_{c}-\left(t_{i}+x_{a, i}\right)\right)} P\left(t_{i}+x_{a, i}\right) \pi_{i}\left(t_{c}\right) \mathrm{d} t_{c}}_{\text {Use (A.3) with } X=0, Y=\rho_{1}, Z=\theta / \eta} \\
& +e^{-\theta \varphi_{1}} \underbrace{\int_{t_{i}+x_{a, i}+\rho_{1}}^{t_{i}+x_{a, i}+\rho_{2}} e^{-\theta \varphi_{2}\left(t_{c}-\left(t_{i}+x_{a, i}\right)\right)} P\left(t_{i}+x_{a, i}\right) \pi_{i}\left(t_{c}\right) \mathrm{d} t_{c}}_{\text {Use (A.3) with } X=\rho_{1}, Y=\rho_{2}, Z=\theta \varphi_{2}} \\
& +e^{-\theta \kappa} \underbrace{\int_{t_{i}+x_{a, i}+\rho_{2}}^{\infty} P\left(t_{i}+x_{a, i}\right) \pi_{i}\left(t_{c}\right) \mathrm{d} t_{c}}_{\text {Use (A.4) with } X=\rho_{2}, Z=0} .
\end{aligned}
$$


The first equality follows from $\widetilde{P}\left(t_{c}\right)=e^{-g \tau^{*}} P\left(t_{c}\right)=e^{-g \tau^{*}} e^{g\left(t_{c}-\left(t_{i}+x_{a, i}\right)\right)} P\left(t_{i}+x_{a, i}\right)$. From (B.6), (B.4) is rewritten as

$$
e^{-\theta-g \tau^{*}}+(g-\lambda) \Lambda\left(0, \rho_{1}, \frac{\theta}{\eta}\right)+e^{-\theta \varphi_{1}}(g-\lambda) \Lambda\left(\rho_{1}, \rho_{2}, \theta \varphi_{2}\right)+e^{-\theta \kappa}\left(g \Lambda\left(\rho_{2}, \tau^{*}-x_{a}^{*}, 0\right)-e^{-\lambda \rho_{2}}\right)=0 .
$$

Likewise, by setting $\theta=0$ and replacing $x_{a}^{*}$ with $x_{b}^{*}$ in (B.7), (B.5) is rewritten as

$$
e^{-g \tau^{*}}+(g-\lambda) \Lambda\left(0, \rho_{1}, 0\right)+(g-\lambda) \Lambda\left(\rho_{1}, \rho_{2}, 0\right)+g \Lambda\left(\rho_{2}, \tau^{*}-x_{b}^{*}, 0\right)-e^{-\lambda \rho_{2}}=0 .
$$

We solve the system of equations (B.1), (B.7), and (B.8) for three unknowns $\tau^{*}, x_{a}^{*}$, and $x_{b}^{*}$.

\section{B.5 Equilibrium with $x_{a}^{*}>x_{b}^{*}$}

Let us obtain $\left(x_{a}^{*}, x_{b}^{*}\right)$ in the equilibrium with $x_{a}^{*}>x_{b}^{*}$. Let $\xi>0$ be a constant such that $x_{a}^{*}=x_{b}^{*}+\xi$. Then we have $\tau^{*}=\eta \kappa+\alpha \xi+x_{b}^{*}, \rho_{1}=\eta \kappa-(1-\alpha) \xi, \rho_{2}=\eta \kappa+\alpha \xi, \varphi_{1}=\alpha(\kappa-(1-\alpha) \xi / \eta)$, and $\varphi_{2}=(1-\alpha) / \eta$. Using these expressions, (B.7) is rewritten as

$$
e^{-g\left(\eta \kappa+\alpha \xi+x_{b}^{*}\right)}=\Omega_{1}(\xi),
$$

where $\Omega_{1}(\xi) \equiv e^{(1-\kappa) \theta}\left(e^{-\lambda(\eta \kappa+\alpha \xi)}-g \Lambda(\eta \kappa+\alpha \xi, \eta \kappa-(1-\alpha) \xi, 0)\right)$

$$
-(g-\lambda) e^{\theta}\left(\begin{array}{l}
\Lambda\left(0, \eta \kappa-(1-\alpha) \xi, \frac{\theta}{\eta}\right) \\
+e^{-\theta \alpha\left(\kappa-\frac{(1-\alpha) \xi}{\eta}\right)} \Lambda\left(\eta \kappa-(1-\alpha) \xi, \eta \kappa+\alpha \xi, \frac{\theta(1-\alpha)}{\eta}\right)
\end{array}\right),
$$

and (B.8) is rewritten as

$$
\begin{array}{cl} 
& e^{-g\left(\eta \kappa+\alpha \xi+x_{b}^{*}\right)}=\Psi_{1}(\xi), \\
\text { where } \quad & \Psi_{1}(\xi) \equiv 1-g \Lambda(0, \eta \kappa+\alpha \xi, 0) .
\end{array}
$$

From (B.9) and (B.11), we have

$$
x_{b}^{*}=-\frac{1}{g} \ln \Omega_{1}(\xi)-\alpha \xi-\eta \kappa,
$$

where $\xi>0$ solves $\Omega_{1}(\xi)=\Psi_{1}(\xi)$. Since $\Omega_{1}^{\prime}(\xi)>0$ for small $\xi>0$ and $\Psi_{1}^{\prime}(\xi)<0$, such a $\xi>0$ exists if $\Omega_{1}(0)$ and $\Psi_{1}(0)$ are close enough and

$$
\Omega_{1}(0)<\Psi_{1}(0) \Longleftrightarrow \hat{x}>x_{A B}
$$

\section{B.6 Equilibrium with $x_{a}^{*} \leq x_{b}^{*}$}

Let us obtain $\left(x_{a}^{*}, x_{b}^{*}\right)$ in the equilibrium with $x_{a}^{*} \leq x_{b}^{*}$. Let $\zeta \geq 0$ be a constant such that $x_{a}^{*}=x_{b}^{*}-\zeta$. Then we have $\tau^{*}=\eta \kappa-\alpha \zeta+x_{b}^{*}, \rho_{1}=\eta \kappa-\alpha \zeta, \rho_{2}=\eta \kappa+(1-\alpha) \zeta, \varphi_{1}=(1-\alpha)(\kappa-\alpha \zeta / \eta)$, and $\varphi_{2}=\alpha / \eta$. Using these expressions, (B.7) is rewritten as

$$
e^{-g\left(\eta \kappa-\alpha \zeta+x_{b}^{*}\right)}=\Omega_{2}(\zeta)
$$


where $\Omega_{2}(\zeta) \equiv e^{(1-\kappa) \theta-\lambda(\eta \kappa+(1-\alpha) \zeta)}$

$$
-(g-\lambda) e^{\theta}\left(\begin{array}{l}
\Lambda\left(0, \eta \kappa-\alpha \zeta, \frac{\theta}{\eta}\right) \\
+e^{-\theta(1-\alpha)\left(\kappa-\frac{\alpha \zeta}{\eta}\right)} \Lambda\left(\eta \kappa-\alpha \zeta, \eta \kappa+(1-\alpha) \zeta, \frac{\theta \alpha}{\eta}\right)
\end{array}\right),
$$

and (B.8) is rewritten as

$$
\begin{array}{cl} 
& e^{-g\left(\eta \kappa-\alpha \zeta+x_{b}^{*}\right)}=\Psi_{2}(\zeta), \\
\text { where } \quad & \Psi_{2}(\zeta) \equiv 1-g \Lambda(0, \eta \kappa-\alpha \zeta, 0) .
\end{array}
$$

From (B.15) and (B.17), we have

$$
x_{b}^{*}=-\frac{1}{g} \ln \Omega_{2}(\zeta)+\alpha \zeta-\eta \kappa,
$$

where $\zeta \geq 0$ solves $\Omega_{2}(\zeta)=\Psi_{2}(\zeta)$. Since $\Omega_{2}^{\prime}(\zeta)<0, \lim _{\zeta \rightarrow \infty} \Omega_{2}(\zeta)=-\infty$, and $\Psi_{2}^{\prime}(\zeta)>0$, such a $\zeta \geq 0$ exists if and only if

$$
\Omega_{2}(0) \geq \Psi_{2}(0) \Longleftrightarrow \hat{x} \leq x_{A B}
$$

\section{Proof of Corollary 3.1}

Rewrite (3.8) as

$$
\hat{x}(\theta)=-\frac{1}{g}(\ln \Sigma(\theta)+\theta)-\eta \kappa, \quad \text { where } \quad \Sigma(\theta) \equiv 1-\frac{g+\frac{\theta}{\eta}}{\lambda+\frac{\theta}{\eta}}\left(1-e^{-\left(\lambda+\frac{\theta}{\eta}\right) \eta \kappa}\right) .
$$

Since $\hat{x}$ is well-defined only if $\Sigma(\theta)>0$, we restrict the parameter values to the ones such that $\Sigma(\theta)>0$. Specifically, we restrict $\theta$ to $[0, \bar{\theta})$, where the upper bound $\bar{\theta}$ is defined in the following lemma.

Lemma C.1. There exists a unique $\bar{\theta}>0$ such that $\Sigma(\theta)>0$ for all $\theta \in[0, \bar{\theta}), \lim _{\theta \rightarrow \bar{\theta}} \Sigma^{\prime}(\theta)<0$, and $\lim _{\theta \rightarrow \bar{\theta}} \Sigma(\theta)=0$.

\section{Proof of Lemma C.1.}

Differentiating $\Sigma(\theta)$,

$$
\Sigma^{\prime}(\theta)=\frac{g-\lambda}{\eta\left(\lambda+\frac{\theta}{\eta}\right)^{2}}\left(1-e^{-\left(\lambda+\frac{\theta}{\eta}\right) \eta \kappa}\right)-\frac{g+\frac{\theta}{\eta}}{\lambda+\frac{\theta}{\eta}} \kappa e^{-\left(\lambda+\frac{\theta}{\eta}\right) \eta \kappa} .
$$

Hence, $\Sigma^{\prime}(\theta)>0$ if and only if $F(\theta)>G(\theta)$, where

$$
F(\theta) \equiv \frac{e^{\left(\lambda+\frac{\theta}{\eta}\right) \eta \kappa}-1}{\eta \kappa} \text { and } \quad G(\theta) \equiv \frac{\left(\lambda+\frac{\theta}{\eta}\right)\left(g+\frac{\theta}{\eta}\right)}{g-\lambda} .
$$

Since $F$ is an exponential function while $G$ is quadratic, $F(\theta)>G(\theta)$ if $\theta$ is large enough (checked by l'Hôspital's rule). Thus, we have

$$
\lim _{\theta \rightarrow \infty} \Sigma^{\prime}(\theta)>0 .
$$


It is easy to check that

$$
\lim _{\theta \rightarrow \infty} \Sigma(\theta)=0 .
$$

From (C.2) and (C.3), there exists some $\theta>0$ such that $\Sigma(\theta)<0$. But since Assumption 1 ensures $\Sigma(0)>0$, there exists $\bar{\theta}>0$ such that $\Sigma(\bar{\theta})=0, \Sigma^{\prime}(\bar{\theta})<0$, and $\Sigma(\theta)>0$ for all $\theta \in[0, \bar{\theta})$. (End of proof of Lemma C.1.)

Lemma C.2. If $\kappa$ is small enough, $\hat{x}$ is $U$-shaped in $\theta$. That is, $\hat{x}=x_{A B}$ for $\theta=0, \hat{x}<x_{A B}$ for small $\theta$, and $\hat{x}>x_{A B}$ for large $\theta$.

\section{Proof of Lemma C.2.}

It is trivial that $\hat{x}=x_{A B}$ for $\theta=0$. From (C.1), we have

$$
\hat{x}^{\prime}(\theta)=-\frac{1}{g}\left(\frac{\Sigma^{\prime}(\theta)}{\Sigma(\theta)}+1\right) .
$$

First, for large $\theta$, we have $\lim _{\theta \rightarrow \bar{\theta}} \hat{x}^{\prime}(\theta)=\infty$ because $\lim _{\theta \rightarrow \bar{\theta}} \Sigma^{\prime}(\theta)<0$ and $\lim _{\theta \rightarrow \bar{\theta}} \Sigma(\theta)=0$. Second, for small $\theta$, we have

$$
\hat{x}^{\prime}(0)=-\frac{1}{g}\left(\frac{\Sigma^{\prime}(0)}{\Sigma(0)}+1\right)=\frac{1}{g \lambda \eta}\left(1-(1-g \eta \kappa) \frac{e^{-\lambda \eta \kappa}}{\Sigma(0)}\right)-\frac{1}{g},
$$

which means $\lim _{\kappa \rightarrow 0} \hat{x}^{\prime}(0)=-1 / g<0$. Thus, $\hat{x}(\theta)$ is U-shaped in $\theta$ if $\kappa$ is small enough. (End of proof of Lemma C.2.)

From Lemma C.2 and Sections B.5 and B.6, we see that $x_{b}^{*}>x_{a}^{*}$ for small $\theta$, whereas $x_{a}^{*}>x_{b}^{*}$ for large $\theta$. Thus, to prove Corollary 3.1, it suffices to prove the following lemma.

Lemma C.3. If $x_{a}^{*}>x_{b}^{*}$ then $x_{b}^{*}>x_{A B}$, and if $x_{a}^{*}<x_{b}^{*}$ then $x_{b}^{*}<x_{A B}$.

\section{Proof of Lemma C.3.}

First, suppose $x_{a}^{*}>x_{b}^{*}$. This corresponds to the case of Section B.5. Thus,

$$
\begin{aligned}
x_{b}^{*} \gtreqless x_{A B} & \Longleftrightarrow-\frac{1}{g} \ln \Psi_{1}(\xi)-\alpha \xi-\eta \kappa \gtreqless-\frac{1}{g} \ln \Psi_{1}(0)-\eta \kappa \\
& \Longleftrightarrow-\frac{1}{g} \ln \frac{\Psi_{1}(\xi)}{\Psi_{1}(0)} \gtreqless \alpha \xi \Longleftrightarrow \frac{\Psi_{1}(\xi)}{\Psi_{1}(0)} \lesseqgtr e^{-g \alpha \xi} \Longleftrightarrow \frac{1-g\left(\frac{1-e^{-\lambda(\eta \kappa+\alpha \xi)}}{\lambda}\right)}{1-g\left(\frac{1-e^{-\lambda \eta \kappa}}{\lambda}\right)} \lesseqgtr e^{-g \alpha \xi} \\
& \Longleftrightarrow F_{1}(\xi) \lesseqgtr G_{1}(\xi), \\
& \text { where } \quad F_{1}(\xi) \equiv \frac{\frac{g}{\lambda} e^{-\lambda \eta \kappa}-\left(\frac{g-\lambda}{\lambda}\right) e^{\lambda \alpha \xi}}{1-g\left(\frac{1-e^{-\lambda \eta \kappa}}{\lambda}\right)} \text { and } G_{1}(\xi) \equiv e^{-(g-\lambda) \alpha \xi} .
\end{aligned}
$$

We have $F_{1}(0)=G_{1}(0)=1, F_{1}^{\prime}(0)<G_{1}^{\prime}(0)<0, F_{1}^{\prime \prime}(\xi)<0$, and $G_{1}^{\prime \prime}(\xi)>0$. Thus, we have $F_{1}(\xi)<G_{1}(\xi)$ for $\xi>0$. From (C.6), this implies $x_{b}^{*}>x_{A B}$. 
Second, suppose $x_{b}^{*}>x_{a}^{*}$. This corresponds to the case of Section B.6. Thus,

$$
\begin{aligned}
x_{b}^{*} \gtreqless x_{A B} & \Longleftrightarrow-\frac{1}{g} \ln \Psi_{2}(\zeta)+\alpha \zeta-\eta \kappa \gtreqless-\frac{1}{g} \ln \Psi_{2}(0)-\eta \kappa \\
& \Longleftrightarrow-\frac{1}{g} \ln \frac{\Psi_{2}(\zeta)}{\Psi_{2}(0)} \gtreqless-\alpha \zeta \Longleftrightarrow \frac{\Psi_{2}(\zeta)}{\Psi_{2}(0)} \lesseqgtr e^{g \alpha \zeta} \Longleftrightarrow \frac{1-g\left(\frac{1-e^{-\lambda(\eta \kappa-\alpha \zeta)}}{\lambda}\right)}{1-g\left(\frac{1-e^{-\lambda \eta \kappa}}{\lambda}\right)} \lesseqgtr e^{g \alpha \zeta} \\
& \Longleftrightarrow F_{2}(\zeta) \lesseqgtr G_{2}(\zeta), \\
& \text { where } \quad F_{2}(\zeta) \equiv \frac{\frac{g}{\lambda} e^{-\lambda \eta \kappa}-\left(\frac{g-\lambda}{\lambda}\right) e^{-\lambda \alpha \zeta}}{1-g\left(\frac{1-e^{-\lambda \eta \kappa}}{\lambda}\right)} \text { and } G_{2}(\zeta) \equiv e^{(g-\lambda) \alpha \zeta}
\end{aligned}
$$

We have $F_{2}(0)=G_{2}(0)=1$ and $F_{2}^{\prime}(0)>G_{2}^{\prime}(0)>0$. Thus, we have $F_{2}(\zeta)>G_{2}(\zeta)$ for $\zeta>0$ small enough. From (C.7), this implies $x_{b}^{*}<x_{A B}$.

\section{Heterogeneous Interim Performance}

\section{D.1 Duration of the bubble}

If the equilibrium holding periods are $\left(x_{G}^{*}, x_{B}^{*}\right)$, the bubble's duration in the symmetric equilibrium is

$$
\tau^{*}=\eta \kappa+\omega x_{G}^{*}+(1-\omega) x_{B}^{*}
$$

The proof is identical to that of Lemma B.1.

\section{D.2 Ranking types}

Depending on the parameter values, there may be an equilibrium in which even the crashed type $G$ are ranked higher than top-ranked type $B$. But there may be an equilibrium such that top type $B$ are ranked higher than some type $G$. We classify these equilibria as different types. Indeed, as shown in the main text, the managers' risk-taking incentives change depending on these types.

Let us define the following six performance levels in the symmetric equilibrium.

- $T G \equiv P\left(t_{0}+\tau^{*}\right)$ : performance of the top $G$

- $F G \equiv P\left(t_{0}+x_{G}^{*}\right):$ performance of the first $G$

- $C G \equiv \widetilde{P}\left(t_{0}+\tau^{*}\right)$ : performance of the crashed $G$

- $T B \equiv e^{-\psi} P\left(t_{0}+\tau^{*}\right)$ : performance of the top $B$

- $F B \equiv e^{-\psi} P\left(t_{0}+x_{B}^{*}\right)$ : performance of the first $B$

- $C B \equiv e^{-\psi} \widetilde{P}\left(t_{0}+\tau^{*}\right)$ : performance of the crashed $B$

In these values, $T G$ is always the largest and $C B$ the smallest. Also, $T G>F G>C G$ and $T B>F B>$ $C B$ hold always. Thus, we need to consider the following six configurations, which are illustrated in Figure 9. 
- Ranking type 1 (R1):

$T G>F G>C G>T B>F B>C B \Longleftrightarrow x_{G}^{*} \geq \tau^{*}-\frac{\psi}{g}, x_{G}^{*} \geq x_{B}^{*}-\frac{\psi}{g}, \tau^{*}<\frac{\psi}{g}$, and $x_{B}^{*}<\frac{\psi}{g}$.

- Ranking type 2 (R2):

$T G>F G \geq T B \geq C G>F B>C B \Longleftrightarrow x_{G}^{*} \geq \tau^{*}-\frac{\psi}{g}, x_{G}^{*} \geq x_{B}^{*}-\frac{\psi}{g}, \tau^{*} \geq \frac{\psi}{g}$, and $x_{B}^{*}<\frac{\psi}{g}$.

- Ranking type 3 (R3):

$T G>F G \geq T B>F B \geq C G>C B \Longleftrightarrow x_{G}^{*} \geq \tau^{*}-\frac{\psi}{g}, x_{G}^{*} \geq x_{B}^{*}-\frac{\psi}{g}, \tau^{*} \geq \frac{\psi}{g}$, and $x_{B}^{*} \geq \frac{\psi}{g}$.

- Ranking type 4 (R4):

$T G>T B>F G>C G>F B>C B \Longleftrightarrow x_{G}^{*}<\tau^{*}-\frac{\psi}{g}, x_{G}^{*} \geq x_{B}^{*}-\frac{\psi}{g}, \tau^{*} \geq \frac{\psi}{g}$, and $x_{B}^{*}<\frac{\psi}{g}$.

- Ranking type 5 (R5):

$T G>T B>F G \geq F B \geq C G>C B \Longleftrightarrow x_{G}^{*}<\tau^{*}-\frac{\psi}{g}, x_{G}^{*} \geq x_{B}^{*}-\frac{\psi}{g}, \tau^{*} \geq \frac{\psi}{g}$, and $x_{B}^{*} \geq \frac{\psi}{g}$.

- Ranking type 6 (R6):

$T G>T B>F B>F G>C G>C B \Longleftrightarrow x_{G}^{*}<\tau^{*}-\frac{\psi}{g}, x_{G}^{*}<x_{B}^{*}-\frac{\psi}{g}, \tau^{*} \geq \frac{\psi}{g}$, and $x_{B}^{*} \geq \frac{\psi}{g}$.

Any equilibrium pair $\left(x_{G}^{*}, x_{B}^{*}\right)$ corresponds to one of these ranking types. Figure 10 illustrates the regions of $\left(x_{G}^{*}, x_{B}^{*}\right)$ corresponding to $(\mathrm{R} 1)-(\mathrm{R} 6)$. The gray area in the figure represents $\left(x_{G}^{*}, x_{B}^{*}\right)$ that are never supported as equilibrium actions. The proof of (D.1) (which is identical to the proof of Lemma B.1) implies that any equilibrium pair $\left(x_{G}^{*}, x_{B}^{*}\right)$ must satisfy

$$
x_{G}^{*} \leq \tau^{*} \leq x_{G}^{*}+\eta \Longleftrightarrow x_{B}^{*}-\left(\frac{1-\kappa}{1-\omega}\right) \eta \leq x_{G}^{*} \leq x_{B}^{*}+\left(\frac{\kappa}{1-\omega}\right) \eta
$$

and

$$
x_{B}^{*} \leq \tau^{*} \leq x_{B}^{*}+\eta \Longleftrightarrow x_{B}^{*}-\left(\frac{\kappa}{\omega}\right) \eta \leq x_{G}^{*} \leq x_{B}^{*}+\left(\frac{1-\kappa}{\omega}\right) \eta .
$$

Combining (D.2) and (D.3) yields a necessary condition for the symmetric equilibrium:

$$
x_{B}^{*}-\min \left\{\frac{1-\kappa}{1-\omega}, \frac{\kappa}{\omega}\right\} \eta<x_{G}^{*}<x_{B}^{*}+\min \left\{\frac{\kappa}{1-\omega}, \frac{1-\kappa}{\omega}\right\} \eta .
$$

The gray area in Figure 10 does not satisfy (D.4).

The symmetric best-response curves in Figure 5 have kinks at the boundaries of regions corresponding to different ranking types. Type $G$ ' best response $R_{G}\left(x_{B}\right)$ has two kinks. In the region of ranking type 1 (bottom left of the figure), the slope of $R_{G}\left(x_{B}\right)$ is less steep. This makes sense. With ranking type 1, even the top type $B$ does not catch up with the crashed type $G$, so that a marginal change in one type's action does not cause a switch of ranks between types (see Figure 9). Thus, a marginal change in $x_{B}$ has a small impact on type $G^{\prime}$ ranks, leading to a mild slope of $R_{G}\left(x_{B}\right)$. In the region of ranking type 2, type $G$ react more aggressively to type $B$ 's action; that is, the slope of $R_{G}\left(x_{B}\right)$ is steeper. This reflects that both types' ranks are clashing with each other (i.e., there are some type $B$ whose ranks are equivalent to those of type $G$ ) so that a marginal change in $x_{B}$ has a large impact on type $G$ 's ranks. ${ }^{21}$ In the region of ranking type $3, R_{G}\left(x_{B}\right)$ is flatter because type $G$ have no clash in ranks with any type $B$, and thus a marginal increase in $x_{B}$ will not cause any over-take by type $B$. In contrast, type $B$ 's best response

\footnotetext{
${ }^{21}$ As seen in Figure 9, an increase in $x_{B}$ makes some type $B$ overtake the crashed type $G$.
} 

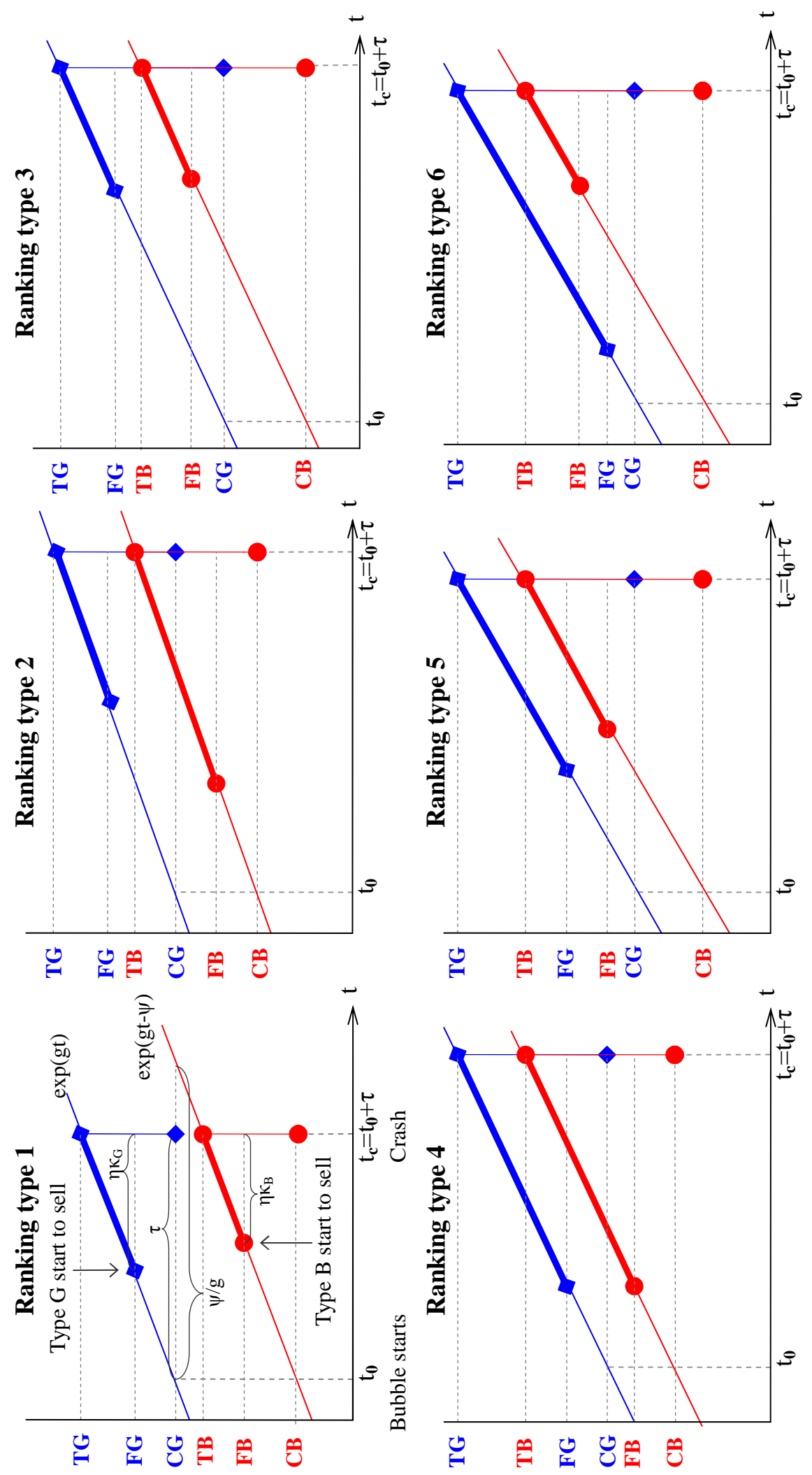

Figure 9: Ranking types 


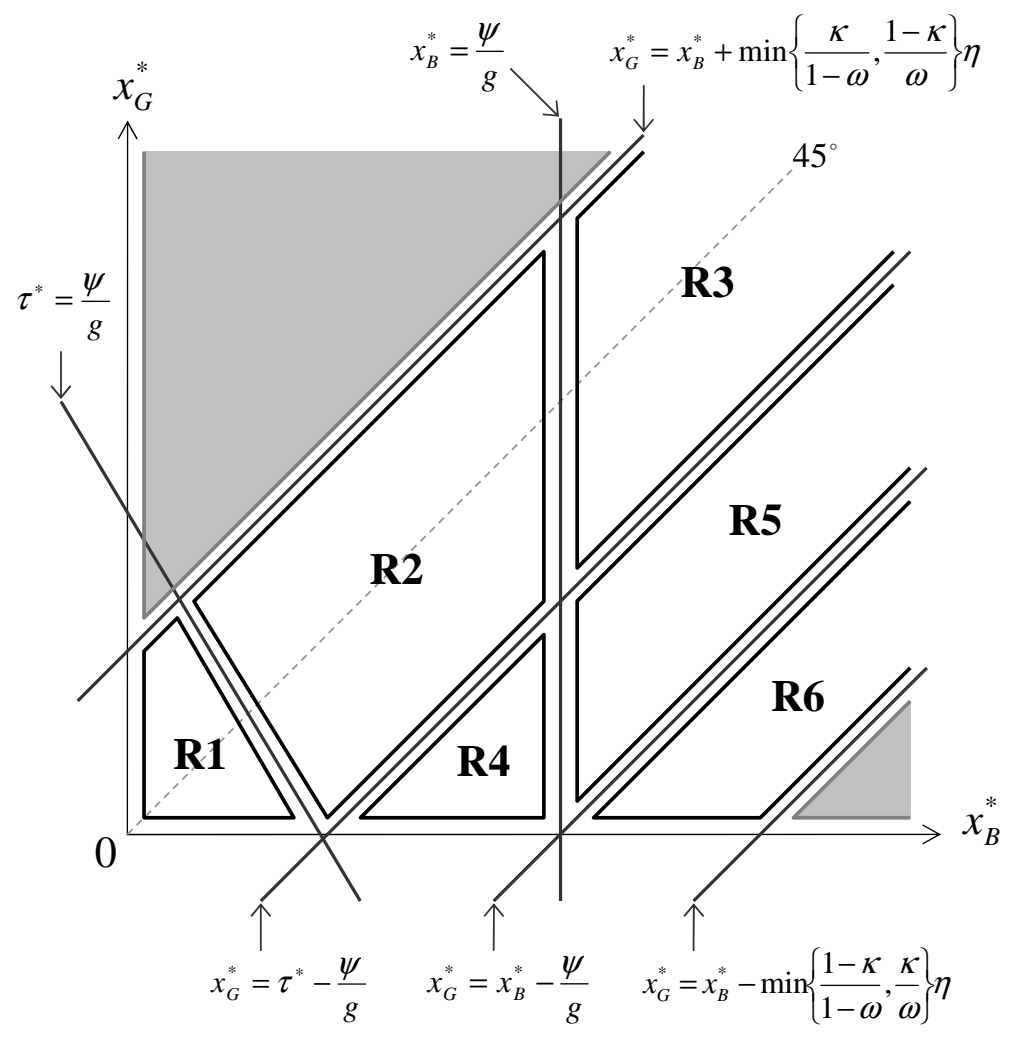

Figure 10: $\left(x_{G}^{*}, x_{B}^{*}\right)$ and ranking types

$R_{B}\left(x_{G}\right)$ has only one kink. It is steeper in the regions of ranking types 2 and 3 because a change in $x_{G}$ has a larger impact on the type $B$ 's ranks. ${ }^{22,23}$

\section{D.3 Type $G$ 's problem}

To solve manager $G$-i's problem, we first rewrite her rank (4.1) as follows.

Lemma D.1. The rank of manager $G-i$ who chooses $x_{G, i}$ is

$$
\gamma_{G, i}= \begin{cases}\omega+(1-\omega) \kappa_{B}^{+} & \text {if } t_{c} \leq t_{i}+x_{G, i}, \\ \frac{\omega}{\eta}\left(t_{c}-\left(t_{i}+x_{G, i}\right)\right) & \text { if } t_{i}+x_{G, i}<t_{c} \leq t_{i}+x_{G, i}+\mu_{1}, \\ \frac{1}{\eta}\left(t_{c}-\left(t_{i}+x_{G, i}\right)\right)-\frac{1-\omega}{\eta} \frac{\psi}{g} & \text { if } t_{i}+x_{G, i}+\mu_{1}<t_{c} \leq t_{i}+x_{G, i}+\mu_{2}, \\ \frac{\omega}{\eta}\left(t_{c}-\left(t_{i}+x_{G, i}\right)\right)+(1-\omega) \kappa_{B} & \text { if } t_{i}+x_{G, i}+\mu_{2}<t_{c} \leq t_{i}+x_{G, i}+\mu_{3}, \\ \omega \kappa_{G} & \text { if } t_{i}+x_{G, i}+\mu_{3}<t_{c} \leq t_{i}+x_{G, i}+\mu_{4}, \\ \omega \kappa_{G}+\frac{1-\omega}{\eta}\left(t_{c}-\left(t_{i}+x_{G, i}\right)\right)-\frac{1-\omega}{\eta} \frac{\psi}{g} & \text { if } t_{i}+x_{G, i}+\mu_{4}<t_{c} \leq t_{i}+x_{G, i}+\mu_{5}, \\ \omega \kappa_{G}+(1-\omega) \kappa_{B}^{+} & \text {if } t_{i}+x_{G, i}+\mu_{5}<t_{c},\end{cases}
$$

${ }^{22}$ For instance, the top type $B$ 's rank in ranking type 1 is a constant $\omega$, whereas in ranking types 2 and 3 , it is $\omega \kappa_{G}$, a function of $x_{G}$.

${ }^{23}$ The type $B$ 's best response has no kink at the boundary between the regions of ranking types 2 and 3 , since the difference between these regions is whether or not $C G$ exceeds $F B$, which is irrelevant to $x_{G}$. 


$$
\begin{aligned}
\text { where } \kappa_{B}^{+} & \equiv \max \left\{0, \min \left\{\frac{1}{\eta}\left(\tau-\frac{\psi}{g}\right), \kappa_{B}\right\}\right\}, \quad \mu_{1} \equiv \min \left\{\frac{\psi}{g}, \eta \kappa_{G}\right\}, \quad \mu_{2} \equiv \min \left\{\frac{\psi}{g}+\eta \kappa_{B}, \eta \kappa_{G}\right\}, \\
\mu_{3} & \equiv \eta \kappa_{G}, \quad \mu_{4} \equiv \mu_{3}+\max \left\{0, \min \left\{\frac{\psi}{g}, \tau\right\}-\eta \kappa_{G}\right\}, \\
\mu_{5} & \equiv \mu_{4}+\max \left\{0, \min \left\{\frac{\psi}{g}+\eta \kappa_{B}, \tau\right\}-\max \left\{\frac{\psi}{g}, \eta \kappa_{G}\right\}\right\} .
\end{aligned}
$$

\section{Proof of Lemma D.1.}

We show that (D.5) nests manager $G$-i's rank in all possible equilibrium outcomes (i.e., six ranking types).

(i) If the equilibrium is of ranking type 1, manager $G$-i's rank is (see Figure 9 for the intuition)

$$
\gamma_{G, i}= \begin{cases}\omega & \text { if } t_{c} \leq t_{i}+x_{G, i} \\ \frac{\omega}{\eta}\left(t_{c}-\left(t_{i}+x_{G, i}\right)\right) & \text { if } t_{c}-\eta \kappa_{G} \leq t_{i}+x_{G, i}<t_{c} \\ \omega \kappa_{G} & \text { if } t_{i}+x_{G, i}<t_{c}-\eta \kappa_{G} .\end{cases}
$$

Since (R1) implies $\kappa_{B}^{+}=0, \mu_{1}=\mu_{2}=\mu_{3}=\eta \kappa_{G}$, and $\mu_{4}=\mu_{5}=\tau$, (D.6) is nested in the first, second, and fifth lines of (D.5).

(ii) If the equilibrium is of ranking type 2, her rank is

$$
\gamma_{G, i}= \begin{cases}\omega+\frac{1-\omega}{\eta}\left(\tau-\frac{\psi}{g}\right) & \text { if } t_{c} \leq t_{i}+x_{G, i} \\ \frac{\omega}{\eta}\left(t_{c}-\left(t_{i}+x_{G, i}\right)\right) & \text { if } t_{c}-\eta \kappa_{G} \leq t_{i}+x_{G, i}<t_{c} \\ \omega \kappa_{G} & \text { if } t_{c}-\frac{\psi}{g} \leq t_{i}+x_{G, i}<t_{c}-\eta \kappa_{G} \\ \omega \kappa_{G}+\frac{1-\omega}{\eta}\left(t_{c}-\left(t_{i}+x_{G, i}+\frac{\psi}{g}\right)\right) & \text { if } t_{i}+x_{G, i}<t_{c}-\frac{\psi}{g}\end{cases}
$$

Since (R2) implies $\kappa_{B}^{+}=(\tau-(\psi / g)) / \eta, \mu_{1}=\mu_{2}=\mu_{3}=\eta \kappa_{G}, \mu_{4}=\psi / g$, and $\mu_{5}=\tau$, (D.7) is nested in the first, second, fifth, and sixth lines of (D.5).

(iii) If the equilibrium is of ranking type 3 , her rank is

$$
\gamma_{G, i}=\left\{\begin{array}{lll}
\omega+(1-\omega) \kappa_{B} & \text { if } t_{c} \leq t_{i}+x_{G, i}, \\
\frac{\omega}{\eta}\left(t_{c}-\left(t_{i}+x_{G, i}\right)\right) & \text { if } t_{c}-\eta \kappa_{G} \leq t_{i}+x_{G, i}<t_{c}, \\
\omega \kappa_{G} & \text { if } t_{c}-\frac{\psi}{g} \leq t_{i}+x_{G, i}<t_{c}-\eta \kappa_{G}, \\
\omega \kappa_{G}+\frac{1-\omega}{\eta}\left(t_{c}-\left(t_{i}+x_{G, i}+\frac{\psi}{g}\right)\right) & \text { if } t_{c}-\left(\frac{\psi}{g}+\eta \kappa_{B}\right) \leq t_{i}+x_{G, i}<t_{c}-\frac{\psi}{g}, \\
\omega \kappa_{G}+(1-\omega) \kappa_{B} & \text { if } t_{i}+x_{G, i}<t_{c}-\left(\frac{\psi}{g}+\eta \kappa_{B}\right) .
\end{array}\right.
$$

Since (R3) implies $\kappa_{B}^{+}=\kappa_{B}, \mu_{1}=\mu_{2}=\mu_{3}=\eta \kappa_{G}, \mu_{4}=\psi / g$, and $\mu_{5}=(\psi / g)+\eta \kappa_{B}$, (D.8) is nested in the first, second, fifth, sixth, and seventh lines of (D.5).

(iv) If the equilibrium is of ranking type 4 , her rank is

$$
\gamma_{G, i}= \begin{cases}\omega+\frac{1-\omega}{\eta}\left(\tau-\frac{\psi}{g}\right) & \text { if } t_{c} \leq t_{i}+x_{G, i}, \\ \frac{\omega}{\eta}\left(t_{c}-\left(t_{i}+x_{G, i}\right)\right) & \text { if } t_{c}-\frac{\psi}{g} \leq t_{i}+x_{G, i}<t_{c}, \\ \frac{\omega}{\eta}\left(t_{c}-\left(t_{i}+x_{G, i}\right)\right)+\frac{1-\omega}{\eta}\left(t_{c}-\left(t_{i}+x_{G, i}+\frac{\psi}{g}\right)\right) & \text { if } t_{c}-\eta \kappa_{G} \leq t_{i}+x_{G, i}<t_{c}-\frac{\psi}{g}, \\ \omega \kappa_{G}+\frac{1-\omega}{\eta}\left(t_{c}-\left(t_{i}+x_{G, i}+\frac{\psi}{g}\right)\right) & \text { if } t_{i}+x_{G, i}<t_{c}-\eta \kappa_{G} .\end{cases}
$$


Since (R4) implies $\kappa_{B}^{+}=(\tau-(\psi / g)) / \eta, \mu_{1}=\psi / g, \mu_{2}=\mu_{3}=\mu_{4}=\eta \kappa_{G}$, and $\mu_{5}=\tau$, (D.9) is nested in the first, second, third, and sixth lines of (D.5).

(v) If the equilibrium is of ranking type 5 , her rank is

$$
\gamma_{G, i}= \begin{cases}\omega+(1-\omega) \kappa_{B} & \text { if } t_{c} \leq t_{i}+x_{G, i}, \\ \frac{\omega}{\eta}\left(t_{c}-\left(t_{i}+x_{G, i}\right)\right) & \text { if } t_{c}-\frac{\psi}{g} \leq t_{i}+x_{G, i}<t_{c}, \\ \frac{\omega}{\eta}\left(t_{c}-\left(t_{i}+x_{G, i}\right)\right)+\frac{1-\omega}{\eta}\left(t_{c}-\left(t_{i}+x_{G, i}+\frac{\psi}{g}\right)\right) & \text { if } t_{c}-\eta \kappa_{G} \leq t_{i}+x_{G, i}<t_{c}-\frac{\psi}{g}, \\ \omega \kappa_{G}+\frac{1-\omega}{\eta}\left(t_{c}-\left(t_{i}+x_{G, i}+\frac{\psi}{g}\right)\right) & \text { if } t_{c}-\left(\frac{\psi}{g}+\eta \kappa_{B}\right) \leq t_{i}+x_{G, i}<t_{c}-\eta \kappa_{G}, \\ \omega \kappa_{G}+(1-\omega) \kappa_{B} & \text { if } t_{i}+x_{G, i}<t_{c}-\left(\frac{\psi}{g}+\eta \kappa_{B}\right) .\end{cases}
$$

Since (R5) implies $\kappa_{B}^{+}=\kappa_{B}, \mu_{1}=\psi / g, \mu_{2}=\mu_{3}=\mu_{4}=\eta \kappa_{G}$, and $\mu_{5}=(\psi / g)+\eta \kappa_{B}$, (D.10) is nested in the first, second, third, sixth, and seventh lines of (D.5).

(vi) If the equilibrium is of ranking type 6 , her rank is

$$
\gamma_{G, i}= \begin{cases}\omega+(1-\omega) \kappa_{B} & \text { if } t_{c} \leq t_{i}+x_{G, i}, \\ \frac{\omega}{\eta}\left(t_{c}-\left(t_{i}+x_{G, i}\right)\right) & \text { if } t_{c}-\frac{\psi}{g} \leq t_{i}+x_{G, i}<t_{c}, \\ \frac{\omega}{\eta}\left(t_{c}-\left(t_{i}+x_{G, i}\right)\right)+\frac{1-\omega}{\eta}\left(t_{c}-\left(t_{i}+x_{G, i}+\frac{\psi}{g}\right)\right) & \text { if } t_{c}-\left(\frac{\psi}{g}+\eta \kappa_{B}\right) \leq t_{i}+x_{G, i}<t_{c}-\frac{\psi}{g}, \\ \frac{\omega}{\eta}\left(t_{c}-\left(t_{i}+x_{G, i}\right)\right)+(1-\omega) \kappa_{B} & \text { if } t_{c}-\eta \kappa_{G} \leq t_{i}+x_{G, i}<t_{c}-\left(\frac{\psi}{g}+\eta \kappa_{B}\right), \\ \omega \kappa_{G}+(1-\omega) \kappa_{B} & \text { if } t_{i}+x_{G, i}<t_{c}-\eta \kappa_{G} .\end{cases}
$$

Since (R6) implies $\kappa_{B}^{+}=\kappa_{B}, \mu_{1}=\psi / g, \mu_{2}=(\psi / g)+\eta \kappa_{B}$, and $\mu_{3}=\mu_{4}=\mu_{5}=\eta \kappa_{G}$, (D.11) is nested in the first, second, third, fourth, and seventh lines of (D.5). (End of proof of Lemma D.1.)

Having characterized $\gamma_{G, i}$, we are ready to solve her maximization problem. Plugging (D.5) into her 
objective function (4.3) and rearranging it so that we can use the formulas of Appendix A,

$$
\begin{aligned}
& V_{G, i}\left(x_{G, i}\right)=e^{-\theta\left(\omega+(1-\omega) \kappa_{B}^{+}\right)-g \tau} \underbrace{\int_{t_{i}}^{t_{i}+x_{G, i}} e^{g\left(t_{c}-\left(t_{i}+x_{G, i}\right)\right)} P\left(t_{i}+x_{G, i}\right) \pi_{i}\left(t_{c}\right) \mathrm{d} t_{c}}_{\text {Use (A.2) with } Z=-g} \\
& +\underbrace{\int_{t_{i}+x_{G, i}}^{t_{i}+x_{G, i}+\mu_{1}} e^{-\theta \frac{\omega}{\eta}\left(t_{c}-\left(t_{i}+x_{G, i}\right)\right)} P\left(t_{i}+x_{G, i}\right) \pi_{i}\left(t_{c}\right) \mathrm{d} t_{c}}_{\text {Use (A.3) with } X=0, Y=\mu_{1}, Z=\theta(\omega / \eta)} \\
& +e^{\theta \frac{1-\omega}{\eta} \frac{\psi}{g}} \underbrace{\int_{t_{i}+x_{G, i}+\mu_{1}}^{t_{i}+x_{G, i}+\mu_{2}} e^{-\frac{\theta}{\eta}\left(t_{c}-\left(t_{i}+x_{G, i}\right)\right)} P\left(t_{i}+x_{G, i}\right) \pi_{i}\left(t_{c}\right) \mathrm{d} t_{c}}_{\text {Use (A.3) with } X=\mu_{1}, Y=\mu_{2}, Z=\theta / \eta} \\
& +e^{-\theta(1-\omega) \kappa_{B}} \underbrace{\int_{t_{i}+x_{G, i}+\mu_{2}}^{t_{i}+x_{G, i}+\mu_{3}} e^{-\theta \frac{\omega}{\eta}\left(t_{c}-\left(t_{i}+x_{G, i}\right)\right)} P\left(t_{i}+x_{G, i}\right) \pi_{i}\left(t_{c}\right) \mathrm{d} t_{c}}_{\text {Use (A.3) with } X=\mu_{2}, Y=\mu_{3}, Z=\theta(\omega / \eta)} \\
& +e^{-\theta \omega \kappa_{G}} \underbrace{\int_{t_{i}+x_{G, i}+\mu_{3}}^{t_{i}+x_{G, i}+\mu_{4}} P\left(t_{i}+x_{G, i}\right) \pi_{i}\left(t_{c}\right) \mathrm{d} t_{c}}_{\text {Use (A.3) with } X=\mu_{3}, Y=\mu_{4}, Z=0} \\
& +e^{-\theta\left(\omega \kappa_{G}-\frac{1-\omega}{\eta} \frac{\psi}{g}\right)} \underbrace{\int_{t_{i}+x_{G, i}+\mu_{4}}^{t_{i}+x_{G, i}+\mu_{5}} e^{-\theta \frac{1-\omega}{\eta}\left(t_{c}-\left(t_{i}+x_{G, i}\right)\right)} P\left(t_{i}+x_{G, i}\right) \pi_{i}\left(t_{c}\right) \mathrm{d} t_{c}}_{\text {Use (A.3) with } X=\mu_{4}, Y=\mu_{5}, Z=\theta(1-\omega) / \eta} \\
& +e^{-\theta\left(\omega \kappa_{G}+(1-\omega) \kappa_{B}^{+}\right)} \underbrace{\int_{t_{i}+x_{G, i}+\mu_{5}}^{\infty} P\left(t_{i}+x_{G, i}\right) \pi_{i}\left(t_{c}\right) \mathrm{d} t_{c}}_{\text {Use (A.4) with } X=\mu_{5}, Z=0} .
\end{aligned}
$$

The first equality follows from $\widetilde{P}\left(t_{c}\right)=e^{-g \tau} P\left(t_{c}\right)=e^{-g \tau} e^{g\left(t_{c}-\left(t_{i}+x_{k, i}\right)\right)} P\left(t_{i}+x_{k, i}\right)$. The type $G^{\prime}$ s symmetric equilibrium condition is obtained as

$$
\begin{gathered}
\left.\frac{\mathrm{d} V_{G, i}\left(x_{G, i}\right)}{\mathrm{d} x_{G, i}}\right|_{x_{G, i}=x_{G}}=0 \\
\Longleftrightarrow e^{-\theta\left(\omega+(1-\omega) \kappa_{B}^{+}\right)-g \tau}+(g-\lambda) \Lambda\left(0, \mu_{1}, \theta \omega / \eta\right)+e^{\theta \frac{1-\omega}{\eta} \frac{\psi}{g}}(g-\lambda) \Lambda\left(\mu_{1}, \mu_{2}, \theta / \eta\right) \\
+e^{-\theta(1-\omega) \kappa_{B}}(g-\lambda) \Lambda\left(\mu_{2}, \mu_{3}, \theta \omega / \eta\right)+e^{-\theta \omega \kappa_{G}}(g-\lambda) \Lambda\left(\mu_{3}, \mu_{4}, 0\right) \\
+e^{-\theta\left(\omega \kappa_{G}-\frac{1-\omega}{\eta} \frac{\psi}{g}\right)}(g-\lambda) \Lambda\left(\mu_{4}, \mu_{5}, \theta(1-\omega) / \eta\right) \\
+e^{-\theta\left(\omega \kappa_{G}+(1-\omega) \kappa_{B}^{+}\right)}\left((g-\lambda) \Lambda\left(\mu_{5}, \tau-x_{G}, 0\right)-e^{-\lambda\left(\tau-x_{G}\right)}\right)=0,
\end{gathered}
$$

where function $\Lambda$ is defined in (3.14). Note that $\tau, \kappa_{G}, \kappa_{B}, \kappa_{B}^{+}$, and $\mu$ s are functions of $x_{G}$ and $x_{B}$. 
Solving (D.13) for $x_{G}$ as a function of $x_{B}$, we have the type $G$ 's symmetric best response, $R_{G}\left(x_{B}\right)$.

\section{D.4 Type $B$ 's problem}

We repeat the procedure of Section D.3 for type $B$.

Lemma D.2. The rank of manager $B$ - $i$ who chooses $x_{B, i}$ is

$$
\gamma_{B, i}= \begin{cases}1 & \text { if } t_{c} \leq t_{i}+x_{B, i}, \\ \frac{\omega}{\eta} \frac{\psi}{g}+\frac{1}{\eta}\left(t_{c}-\left(t_{i}+x_{B, i}\right)\right) & \text { if } t_{i}+x_{B, i}<t_{c} \leq t_{i}+x_{B, i}+\nu_{1}, \\ \omega \kappa_{G}+\frac{1-\omega}{\eta}\left(t_{c}-\left(t_{i}+x_{B, i}\right)\right) & \text { if } t_{i}+x_{B, i}+\nu_{1}<t_{c} \leq t_{i}+x_{B, i}+\nu_{2}, \\ \omega+\frac{1-\omega}{\eta}\left(t_{c}-\left(t_{i}+x_{B, i}\right)\right) & \text { if } t_{i}+x_{B, i}+\nu_{2}<t_{c} \leq t_{i}+x_{B, i}+\nu_{3}, \\ \frac{\omega}{\eta}\left(t_{c}-\left(t_{i}+x_{B, i}\right)+\frac{\psi}{g}\right)+(1-\omega) \kappa_{B} & \text { if } t_{i}+x_{B, i}+\nu_{3}<t_{c} \leq t_{i}+x_{B, i}+\nu_{4}, \\ \omega \kappa_{G}+(1-\omega) \kappa_{B} & \text { if } t_{i}+x_{B, i}+\nu_{4}<t_{c} \leq t_{i}+x_{B, i}+\nu_{5}, \\ \omega+(1-\omega) \kappa_{B} & \text { if } t_{i}+x_{B, i}+\nu_{5}<t_{c},\end{cases}
$$

where $\begin{aligned} \nu_{1} & \equiv \eta \kappa_{B}^{++}, \quad \nu_{2} \equiv \eta \kappa_{B}^{+}, \quad \nu_{3} \equiv \eta \kappa_{B}, \quad \nu_{4} \equiv \nu_{3}+\max \left\{0, \eta \kappa_{G}-\left(\frac{\psi}{g}+\eta \kappa_{B}\right)\right\}, \\ \nu_{5} & \equiv \nu_{4}+\max \left\{0, \tau-\max \left\{\frac{\psi}{g}+\eta \kappa_{B}, \eta \kappa_{G}\right\}\right\}, \quad \kappa_{B}^{++} \equiv \frac{1}{\eta} \max \left\{0, \min \left\{\eta \kappa_{G}, \frac{\psi}{g}+\eta \kappa_{B}\right\}-\frac{\psi}{g}\right\} .\end{aligned}$

\section{Proof of Lemma D.2.}

We show that (D.14) nests manager $B$-i's rank in all possible equilibrium outcomes (six ranking types).

(i) If the equilibrium is of ranking type 1 , manager $B$-i's rank is

$$
\gamma_{B, i}= \begin{cases}1 & \text { if } t_{c} \leq t_{i}+x_{B, i} \\ \omega+\frac{1-\omega}{\eta}\left(t_{c}-\left(t_{i}+x_{B, i}\right)\right) & \text { if } t_{c}-\eta \kappa_{B} \leq t_{i}+x_{B, i}<t_{c} \\ \omega+(1-\omega) \kappa_{B} & \text { if } t_{i}+x_{B, i}<t_{c}-\eta \kappa_{B} .\end{cases}
$$

Since (R1) implies $\kappa_{B}^{++}=\kappa_{B}^{+}=0, \nu_{1}=\nu_{2}=0$, and $\nu_{3}=\nu_{4}=\nu_{5}=\eta \kappa_{B}$, (D.15) is nested in the first, fourth, and seventh lines of (D.14).

(ii) If the equilibrium is of ranking type 2 , her rank is

$$
\gamma_{B, i}=\left\{\begin{array}{lll}
1 & \text { if } \quad t_{c} \leq t_{i}+x_{B, i} \\
\omega \kappa_{G}+\frac{1-\omega}{\eta}\left(t_{c}-\left(t_{i}+x_{B, i}\right)\right) & \text { if } \quad t_{c}-\left(\tau-\frac{\psi}{g}\right) \leq t_{i}+x_{B, i}<t_{c}, \\
\omega+\frac{1-\omega}{\eta}\left(t_{c}-\left(t_{i}+x_{B, i}\right)\right) & \text { if } \quad t_{c}-\eta \kappa_{B} \leq t_{i}+x_{B, i}<t_{c}-\left(\tau-\frac{\psi}{g}\right), \\
\omega+(1-\omega) \kappa_{B} & \text { if } \quad t_{i}+x_{B, i}<t_{c}-\eta \kappa_{B} .
\end{array}\right.
$$

Since (R2) implies $\kappa_{B}^{++}=0, \kappa_{B}^{+}=(\tau-(\psi / g)) / \eta, \nu_{1}=0, \nu_{2}=\tau-(\psi / g)$, and $\nu_{3}=\nu_{4}=\nu_{5}=\eta \kappa_{B}$, (D.16) is nested in the first, third, fourth, and seventh lines of (D.14). 
(iii) If the equilibrium is of ranking type 3 , her rank is

$$
\gamma_{B, i}= \begin{cases}1 & \text { if } \quad t_{c} \leq t_{i}+x_{B, i} \\ \omega \kappa_{G}+\frac{1-\omega}{\eta}\left(t_{c}-\left(t_{i}+x_{B, i}\right)\right) & \text { if } t_{c}-\eta \kappa_{B} \leq t_{i}+x_{B, i}<t_{c} \\ \omega \kappa_{G}+(1-\omega) \kappa_{B} & \text { if } t_{c}-\left(\tau-\frac{\psi}{g}\right) \leq t_{i}+x_{B, i}<t_{c}-\eta \kappa_{B} \\ \omega+(1-\omega) \kappa_{B} & \text { if } t_{i}+x_{B, i}<t_{c}-\left(\tau-\frac{\psi}{g}\right) .\end{cases}
$$

Since (R3) implies $\kappa_{B}^{++}=0, \kappa_{B}^{+}=\kappa_{B}, \nu_{1}=0, \nu_{2}=\nu_{3}=\nu_{4}=\eta \kappa_{B}$, and $\nu_{5}=\tau-(\psi / g),(\mathrm{D} .17)$ is nested in the first, third, sixth, and seventh lines of (D.14).

(iv) If the equilibrium is of ranking type 4 , her rank is

$$
\gamma_{B, i}= \begin{cases}1 & \text { if } t_{c} \leq t_{i}+x_{B, i}, \\ \frac{\omega}{\eta}\left(t_{c}-\left(t_{i}+x_{B, i}-\frac{\psi}{g}\right)\right)+\frac{1-\omega}{\eta}\left(t_{c}-\left(t_{i}+x_{B, i}\right)\right) & \text { if } t_{c}-\left(\eta \kappa_{G}-\frac{\psi}{g}\right) \leq t_{i}+x_{B, i}<t_{c}, \\ \omega \kappa_{G}+\frac{1-\omega}{\eta}\left(t_{c}-\left(t_{i}+x_{B, i}\right)\right) & \text { if } t_{c}-\left(\tau-\frac{\psi}{g}\right) \leq t_{i}+x_{B, i}<t_{c}-\left(\eta \kappa_{G}-\frac{\psi}{g}\right), \\ \omega+\frac{1-\omega}{\eta}\left(t_{c}-\left(t_{i}+x_{B, i}\right)\right) & \text { if } t_{c}-\eta \kappa_{B}<t_{i}+x_{B, i} \leq t_{c}-\left(\tau-\frac{\psi}{g}\right), \\ \omega+(1-\omega) \kappa_{B} & \text { if } t_{i}+x_{B, i}<t_{c}-\eta \kappa_{B} .\end{cases}
$$

Since (R4) implies $\kappa_{B}^{++}=\left(\eta \kappa_{G}-(\psi / g)\right) / \eta, \kappa_{B}^{+}=(\tau-(\psi / g)) / \eta, \nu_{1}=\eta \kappa_{G}-(\psi / g), \nu_{2}=\tau-(\psi / g)$, and $\nu_{3}=\nu_{4}=\nu_{5}=\eta \kappa_{B}$, (D.18) is nested in the first, second, third, fourth, and seventh lines of (D.14).

(v) If the equilibrium is of ranking type 5 , her rank is

$$
\gamma_{B, i}= \begin{cases}1 & \text { if } t_{c} \leq t_{i}+x_{B, i}, \\ \frac{\omega}{\eta}\left(t_{c}-\left(t_{i}+x_{B, i}-\frac{\psi}{g}\right)\right)+\frac{1-\omega}{\eta}\left(t_{c}-\left(t_{i}+x_{B, i}\right)\right) & \text { if } t_{c}-\left(\eta \kappa_{G}-\frac{\psi}{g}\right) \leq t_{i}+x_{B, i}<t_{c}, \\ \omega \kappa_{G}+\frac{1-\omega}{\eta}\left(t_{c}-\left(t_{i}+x_{B, i}\right)\right) & \text { if } t_{c}-\eta \kappa_{B} \leq t_{i}+x_{B, i}<t_{c}-\left(\eta \kappa_{G}-\frac{\psi}{g}\right), \\ \omega \kappa_{G}+(1-\omega) \kappa_{B} & \text { if } t_{c}-\left(\tau-\frac{\psi}{g}\right) \leq t_{i}+x_{B, i}<t_{c}-\eta \kappa_{B}, \\ \omega+(1-\omega) \kappa_{B} & \text { if } t_{i}+x_{B, i}<t_{c}-\left(\tau-\frac{\psi}{g}\right) .\end{cases}
$$

Since (R5) implies $\kappa_{B}^{++}=\left(\eta \kappa_{G}-(\psi / g)\right) / \eta, \kappa_{B}^{+}=\kappa_{B}, \nu_{1}=\eta \kappa_{G}-(\psi / g), \nu_{2}=\nu_{3}=\nu_{4}=\eta \kappa_{B}$, and $\nu_{5}=\tau-(\psi / g),($ D.19) is nested in the first, second, third, sixth, and seventh lines of (D.14).

(vi) If the equilibrium is of ranking type 6 , her rank is

$$
\gamma_{B, i}= \begin{cases}1 & \text { if } t_{c} \leq t_{i}+x_{B, i} \\ \frac{\omega}{\eta}\left(t_{c}-\left(t_{i}+x_{B, i}-\frac{\psi}{g}\right)\right)+\frac{1-\omega}{\eta}\left(t_{c}-\left(t_{i}+x_{B, i}\right)\right) & \text { if } t_{c}-\eta \kappa_{B} \leq t_{i}+x_{B, i}<t_{c} \\ \frac{\omega}{\eta}\left(t_{c}-\left(t_{i}+x_{B, i}-\frac{\psi}{g}\right)\right)+(1-\omega) \kappa_{B} & \text { if } t_{c}-\left(\eta \kappa_{G}-\frac{\psi}{g}\right) \leq t_{i}+x_{G, i}<t_{c}-\eta \kappa_{B} \\ \omega \kappa_{G}+(1-\omega) \kappa_{B} & \text { if } t_{c}-\left(\tau-\frac{\psi}{g}\right) \leq t_{i}+x_{B, i}<t_{c}-\left(\eta \kappa_{G}-\frac{\psi}{g}\right), \\ \omega+(1-\omega) \kappa_{B}, & \text { if } t_{i}+x_{B, i}<t_{c}-\left(\tau-\frac{\psi}{g}\right)\end{cases}
$$

Since (R6) implies $\kappa^{++}=\kappa_{B}^{+}=\kappa_{B}, \nu_{1}=\nu_{2}=\nu_{3}=\eta \kappa_{B}, \nu_{4}=\eta \kappa_{G}-(\psi / g)$, and $\nu_{5}=\tau-(\psi / g)$, (D.20) is nested in the first, second, fifth, sixth, and seventh lines of (D.14). (End of proof of Lemma D.2.) 
Plugging (D.14) into her objective function (4.3) and rearranging, we have

$$
\begin{aligned}
& V_{B, i}\left(x_{B, i}\right)=e^{-\theta-g \tau} \underbrace{\int_{t_{i}}^{t_{i}+x_{B, i}} e^{g\left(t_{c}-\left(t_{i}+x_{B, i}\right)\right)} P\left(t_{i}+x_{B, i}\right) \pi_{i}\left(t_{c}\right) \mathrm{d} t_{c}}_{\text {Use (A.2) with } Z=-g} \\
& +e^{-\theta \frac{\omega}{\eta} \frac{\psi}{g}} \underbrace{\int_{t_{i}+x_{B, i}}^{t_{i}+x_{B, i}+\nu_{1}} e^{-\frac{\theta}{\eta}\left(t_{c}-\left(t_{i}+x_{B, i}\right)\right)} P\left(t_{i}+x_{B, i}\right) \pi_{i}\left(t_{c}\right) \mathrm{d} t_{c}}_{\text {Use (A.3) with } X=0, Y=\nu_{1}, Z=\theta / \eta} \\
& +e^{-\theta \omega \kappa_{G}} \underbrace{\int_{t_{i}+x_{B, i}+\nu_{1}}^{t_{i}+x_{B, i}+\nu_{2}} e^{-\theta \frac{1-\omega}{\eta}\left(t_{c}-\left(t_{i}+x_{B, i}\right)\right)} P\left(t_{i}+x_{B, i}\right) \pi_{i}\left(t_{c}\right) \mathrm{d} t_{c}}_{\text {Use (A.3) with } X=\nu_{1}, Y=\nu_{2}, Z=\theta(1-\omega) / \eta} \\
& +e^{-\theta \omega} \underbrace{\int_{t_{i}+x_{B, i}+\nu_{2}}^{t_{i}+x_{B, i}+\nu_{3}} e^{-\theta \frac{1-\omega}{\eta}\left(t_{c}-\left(t_{i}+x_{B, i}\right)\right)} P\left(t_{i}+x_{B, i}\right) \pi_{i}\left(t_{c}\right) \mathrm{d} t_{c}}_{\text {Use (A.3) with } X=\nu_{2}, Y=\nu_{3}, Z=\theta(1-\omega) / \eta} \\
& +e^{-\theta\left(\frac{\omega}{\eta} \frac{\psi}{g}+(1-\omega) \kappa_{B}\right)} \underbrace{\int_{t_{i}+x_{B, i}+\nu_{3}}^{t_{i}+x_{B, i}+\nu_{4}} e^{-\theta \frac{\omega}{\eta}\left(t_{c}-\left(t_{i}+x_{B, i}\right)\right)} P\left(t_{i}+x_{B, i}\right) \pi_{i}\left(t_{c}\right) \mathrm{d} t_{c}}_{\text {Use (A.3) with } X=\nu_{3}, Y=\nu_{4}, Z=\theta(\omega / \eta)} \\
& +e^{-\theta\left(\omega \kappa_{G}+(1-\omega) \kappa_{B}\right)} \underbrace{\int_{t_{i}+x_{B, i}+\nu_{4}}^{t_{i}+x_{B, i}+\nu_{5}} P\left(t_{i}+x_{B, i}\right) \pi_{i}\left(t_{c}\right) \mathrm{d} t_{c}}_{\text {Use (A.3) with } X=\nu_{4}, Y=\nu_{5}, Z=0} \\
& +e^{-\theta\left(\omega+(1-\omega) \kappa_{B}\right)} \underbrace{\int_{t_{i}+x_{B, i}+\nu_{5}}^{\infty} P\left(t_{i}+x_{B, i}\right) \pi_{i}\left(t_{c}\right) \mathrm{d} t_{c}}_{\text {Use (A.4) with } X=\nu_{5}, Z=0} .
\end{aligned}
$$

The symmetric equilibrium condition for type $B$ is

$$
\begin{gathered}
\left.\frac{\mathrm{d} V_{B, i}\left(x_{B, i}\right)}{\mathrm{d} x_{B, i}}\right|_{x_{B, i}=x_{B}}=0 \\
\Longleftrightarrow e^{-\theta-g \tau}+e^{-\theta \frac{\omega}{\eta} \frac{\psi}{g}}(g-\lambda) \Lambda\left(0, \nu_{1}, \theta / \eta\right)+e^{-\theta \omega \kappa_{G}}(g-\lambda) \Lambda\left(\nu_{1}, \nu_{2}, \theta(1-\omega) / \eta\right) \\
+e^{-\theta \omega}(g-\lambda) \Lambda\left(\nu_{2}, \nu_{3}, \theta(1-\omega) / \eta\right)+e^{-\theta\left(\frac{\omega}{\eta} \frac{\psi}{g}+(1-\omega) \kappa_{B}\right)}(g-\lambda) \Lambda\left(\nu_{3}, \nu_{4}, \theta \omega / \eta\right) \\
+e^{-\theta\left(\omega \kappa_{G}+(1-\omega) \kappa_{B}\right)}(g-\lambda) \Lambda\left(\nu_{4}, \nu_{5}, 0\right) \\
+e^{-\theta\left(\omega+(1-\omega) \kappa_{B}\right)}\left((g-\lambda) \Lambda\left(\nu_{5}, \tau-x_{B}, 0\right)-e^{-\lambda\left(\tau-x_{B}\right)}\right)=0 .
\end{gathered}
$$

Note that $\tau, \kappa_{G}, \kappa_{B}$, and $\xi \mathrm{s}$ are functions of $x_{G}$ and $x_{B}$. Solving (D.22) for $x_{B}$ as a function of $x_{G}$, we have the type $B$ 's symmetric best response, $R_{B}\left(x_{G}\right)$. 


\section{E Random Evaluation Time}

Plugging the manager's rank (5.1)-(5.3) into her objective function (5.4) and noting that $t_{0}+x^{* *}=t_{c}-\eta \kappa$ holds identically, we have

$$
\begin{aligned}
V_{i}\left(x_{i}\right)=\int_{t_{i}}^{t_{i}+x_{i}}\left\{\begin{array}{c}
\int_{0}^{t_{c}-\eta \kappa} e^{-\theta} P\left(t_{e}\right) \mathrm{d} H\left(t_{e}\right) \\
+\int_{t_{c}-\eta \kappa}^{t_{c}} e^{-\theta\left((1-\kappa)+\frac{1}{\eta}\left(t_{c}-t_{e}\right)\right)} P\left(t_{e}\right) \mathrm{d} H\left(t_{e}\right) \\
+\int_{t_{c}}^{\infty} e^{-\theta} \widetilde{P}\left(t_{c}\right) \mathrm{d} H\left(t_{e}\right)
\end{array}\right\} \pi_{i}\left(t_{c}\right) \mathrm{d} t_{c} \\
+\int_{t_{i}+x_{i}}^{t_{i}+x_{i}+\eta \kappa}\left\{\begin{array}{c}
\int_{0}^{t_{c}-\eta \kappa} e^{-\theta} P\left(t_{e}\right) \mathrm{d} H\left(t_{e}\right) \\
+\int_{t_{c}-\eta \kappa}^{t_{i}+x_{i}} e^{-\theta\left((1-\kappa)+\frac{1}{\eta}\left(t_{c}-t_{e}\right)\right)} P\left(t_{e}\right) \mathrm{d} H\left(t_{e}\right) \\
+\int_{t_{i}+x_{i}}^{t_{c}} e^{-\theta\left((1-\kappa)+\frac{1}{\eta}\left(t_{c}-\left(t_{i}+x_{i}\right)\right)\right)} P\left(t_{i}+x_{i}\right) \mathrm{d} H\left(t_{e}\right) \\
+\int_{t_{c}}^{\infty} e^{-\theta \frac{1}{\eta}\left(t_{c}-\left(t_{i}+x_{i}\right)\right)} P\left(t_{i}+x_{i}\right) \mathrm{d} H\left(t_{e}\right) \\
\int_{0}^{t_{i}+x_{i}} e^{-\theta} P\left(t_{e}\right) \mathrm{d} H\left(t_{e}\right) \\
+\int_{t_{i}+x_{i}}^{t_{c}} e^{-\theta} P\left(t_{i}+x_{i}\right) \mathrm{d} H\left(t_{e}\right) \\
+\int_{t_{c}}^{\infty} e^{-\theta \kappa} P\left(t_{i}+x_{i}\right) \mathrm{d} H\left(t_{e}\right)
\end{array}\right\} \pi_{i}\left(t_{c}\right) \mathrm{d} t_{c} .
\end{aligned} \pi_{i}\left(t_{c}\right) \mathrm{d} t_{c}
$$


After some algebra, we can rearrange (E.1) so that we can use the formulas of Appendix A (with $g$ replaced by $g-\phi$ ) as follows.

$$
\begin{aligned}
& V_{i}\left(x_{i}\right)=A_{0}+\left(A_{1}+e^{-\theta-g \tau}\right) \underbrace{\int_{t_{i}}^{t_{i}+x_{i}} e^{(g-\phi) t_{c}} \pi_{i}\left(t_{c}\right) \mathrm{d} t_{c}}_{\text {Use (A.2) with } Z=-(g-\phi)} \\
& +A_{2} \underbrace{\int_{t_{i}+x_{i}}^{t_{i}+x_{i}+\eta \kappa} e^{(g-\phi) t_{c}} \pi_{i}\left(t_{c}\right) \mathrm{d} t_{c}}_{\text {Use (A.3) with } X=0, Y=\eta \kappa, Z=-(g-\phi) .} \\
& +A_{3} \underbrace{\int_{t_{i}+x_{i}}^{t_{i}+x_{i}+\eta \kappa} e^{-\frac{\theta}{\eta}\left(t_{c}-\left(t_{i}+x_{i}\right)\right)} e^{(g-\phi)\left(t_{i}+x_{i}\right)} \pi_{i}\left(t_{c}\right) \mathrm{d} t_{c}}_{\text {Use (A.3) with } X=0, Y=\eta \kappa, Z=\frac{\theta}{\eta} .} \\
& +A_{4} \underbrace{\int_{t_{i}+x_{i}}^{t_{i}+x_{i}+\eta \kappa} e^{-\left(\phi+\frac{\theta}{\eta}\right)\left(t_{c}-\left(t_{i}+x_{i}\right)\right)} e^{(g-\phi)\left(t_{i}+x_{i}\right)} \pi_{i}\left(t_{c}\right) \mathrm{d} t_{c}}_{\text {User (A.3) with } X=0, Y=\eta \kappa, Z=\phi+\frac{\theta}{\eta} .} \\
& +A_{5} \underbrace{\int_{t_{i}+x_{i}+\eta \kappa}^{\infty} e^{(g-\phi)\left(t_{i}+x_{i}\right)} \pi_{i}\left(t_{c}\right) \mathrm{d} t_{c}}_{\text {Use (A.4) with } X=\eta \kappa, Z=0 .} \\
& +A_{6} \underbrace{\int_{t_{i}+x_{i}+\eta \kappa}^{\infty} e^{-\phi\left(t_{c}-\left(t_{i}+x_{i}\right)\right)} e^{(g-\phi)\left(t_{i}+x_{i}\right)} \pi_{i}\left(t_{c}\right) \mathrm{d} t_{c}}_{\text {Use (A.4) with } X=\eta \kappa, Z=\phi}
\end{aligned}
$$

where $\quad A_{0} \equiv-\frac{\phi e^{-\theta}}{g-\phi}, A_{1} \equiv \frac{\phi}{g-\phi} e^{-\theta-(g-\phi) \eta \kappa}+\frac{\phi e^{-\theta(1-\kappa)}}{g-\phi+\frac{\theta}{\eta}}\left(1-e^{-\left(g-\phi+\frac{\theta}{\eta}\right) \eta \kappa}\right)$

$$
\begin{aligned}
& A_{2} \equiv \frac{\phi e^{-\theta-(g-\phi) \eta \kappa}}{g-\phi}-\frac{\phi e^{-\theta(1-\kappa)-\left(g-\phi+\frac{\theta}{\eta}\right) \eta \kappa}}{g-\phi+\frac{\theta}{\eta}}, A_{3} \equiv \frac{g+\frac{\theta}{\eta}}{g-\phi+\frac{\theta}{\eta}} e^{-\theta(1-\kappa)} \\
& A_{4} \equiv 1-e^{-\theta(1-\kappa)}, A_{5} \equiv \frac{g}{g-\phi} e^{-\theta}, A_{6} \equiv e^{-\theta \kappa}-e^{-\theta}
\end{aligned}
$$

The symmetric equilibrium holding period $x^{* *}$ is determined by

$$
\left.\frac{\mathrm{d} V_{i}\left(x_{i}\right)}{\mathrm{d} x_{i}}\right|_{x_{i}=x^{* *}}=0 \Longleftrightarrow e^{-g\left(\eta \kappa+x^{* *}\right)}=K
$$

where

$$
\begin{aligned}
K & \equiv\left(\frac{g}{g-\phi} e^{-\lambda \eta \kappa}-e^{-(\lambda+\phi) \eta \kappa}\right)-\phi\left(\frac{e^{\theta \kappa}-e^{-\lambda \eta \kappa}}{g-\phi+\frac{\theta}{\eta}}+\frac{e^{-\lambda \eta \kappa}}{g-\phi}\right) \\
& -e^{\theta \kappa}(g-\phi-\lambda)\left(\frac{g+\frac{\theta}{\eta}}{g-\phi+\frac{\theta}{\eta}} \Lambda\left(0, \eta \kappa, \frac{\theta}{\eta}\right)-\Lambda\left(0, \eta \kappa, \phi+\frac{\theta}{\eta}\right)\right)+e^{\theta}\left(1-\left(g+\frac{\theta}{\eta}\right) \Lambda\left(0, \eta \kappa, \phi+\frac{\theta}{\eta}\right)\right)
\end{aligned}
$$

is a constant. Rearranging (E.4) yields (5.5). 


\section{References}

[1] Abreu, D., and M. Brunnermeier, 2003, "Bubbles and Crashes," Econometrica, 71, 173-204.

[2] Allen, F., and D. Gale, 2000, "Bubbles and Crises," Economic Journal, 110, 236-255.

[3] Allen, F., and G. Gorton, 1993, "Churning Bubbles," Review of Economic Studies, $60,813-836$.

[4] Basak, S., and D. Makarov, 2012, "Strategic Asset Allocation in Money Management," Journal of Finance, forthcoming.

[5] Brown, K., Harlow, W. V. and L. Starks, 1996, "Of Tournaments and Temptations: An Analysis of Managerial Incentives in the Mutual Fund Industry," Journal of Finance, 51, 85-110.

[6] Brunnermeier, M., and S. Nagel, 2004, "Hedge Funds and the Technology Bubble," Journal of Finance, 59, 2013-2040.

[7] Brunnermeier, M., and M. Oehmke, 2013, "Bubbles, Financial Crises, and Systemic Risk," Handbook of the Economics of Finance.

[8] Busse, J., 2001, "Another Look at Mutual Fund Tournaments," Journal of Financial and Quantitative Analysis, 36, 53-73.

[9] Cabral, L., 2003, "R\&D Competition When Firms Choose Variance," Journal of Economics \& Management Strategy, 12, 139-150.

[10] Chevalier, J., and G. Ellison, 1997, "Risk Taking by Mutual Funds as a Response to Incentives," Journal of Political Economy, 105, 1167-1200.

[11] — 1999, "Career Concerns of Mutual Fund Managers," Quarterly Journal of Economics, 114, 389-432.

[12] Dass, N., Massa, M., and Patgiri, R., 2008, "Mutual Funds and Bubbles: The Surprising Role of Contractual Incentives," Review of Financial Studies, 21, 51-99.

[13] Deli, D., 2002, "Mutual Fund Advisory Contracts: An Empirical Investigation," Journal of Finance, 57, 109-134. 
[14] DeMarzo, P., Kaniel, R. and I. Kremer, 2008, "Relative Wealth Concerns and Financial Bubbles," Review of Financial Studies, 21, 19-50.

[15] Doblas-Madrid, A., 2012, "A Robust Model of Bubbles with Multidimensional Uncertainty," Econometrica 80, 1245-1293.

[16] Goriaev, A., Palomino, F. and A. Prat, 2003, "Mutual Fund Tournament: Risk Taking Incentives Induced by Ranking Objectives," Working Paper, New Economic School, HEC School of Management and London School of Economics.

[17] Greenwood, R., and S. Nagel, 2009, "Inexperienced Investors and Bubbles," Journal of Financial Economics, 93, 239-258.

[18] Griffin, J., Harris, J., Shu, T., and S. Topaloglu, 2011, "Who Drove and Burst the Tech Bubble?," Journal of Finance, 66, 1251-1290.

[19] Hong, H., Scheinkman, J. and W. Xiong, 2008, "Advisors and Asset Prices: A Model of the Origins of Bubbles," Journal of Financial Economics, 89, 268-287.

[20] Hvide, H., 2002, "Tournament Rewards and Risk Taking," Journal of Labor Economics, 20, 877-898.

[21] Investment Company Institute, 2014, 2014 Investment Company Fact Book, 54th Edition.

[22] Isaac, M., and D. James, 2000, "Asset Markets: How They Are Affected by Tournament Incentives for Individuals," American Economic Review, 90, 995-1004.

[23] Koski, J. L., and J. Pontiff, 1999, "How Are Derivatives Used? Evidence from the Mutual Fund Industry," Journal of Finance, 54, 791-816.

[24] Palomino, F., 2005, "Relative Performance Objectives in Financial Markets," Journal of Financial Intermediation, 14, 351-375.

[25] Sirri, E. R., and P. Tufano, 1998, "Costly Search and Mutual Fund Flows," Journal of Finance, 53, 1589-1622. 